Available online at www.sciencedirect.com

science $\boldsymbol{d}$ Direct.

\title{
Formation and growth of tracks in nuclear track materials
}

\author{
D. Nikezic ${ }^{\mathrm{a}, \mathrm{b}}$, K.N. Yu ${ }^{\mathrm{b}, *}$ \\ ${ }^{a}$ Faculty of Science, University of Kragujevac, Kragujevac, Serbia and Monte Negro \\ ${ }^{\mathrm{b}}$ Department of Physics and Materials Science, City University of Hong Kong, Tat Chee Avenue, Kowloon Tong, \\ Kowloon, Hong Kong
}

Accepted 14 July 2004

Available online 18 September 2004

\begin{abstract}
This paper reviews some aspects of solid-state nuclear track detectors (SSNTDs) and their applications in the radon and other research fields. Several geometrical models for the track growth given in the literature are described and compared. It is found that different models give close results for the dimensions of track openings.

One of the main parameters that govern track formation is the bulk etch rate $V_{\mathrm{b}}$. Dependences of $V_{\mathrm{b}}$ on different parameters such as the preparation procedures, etching conditions, irradiation before etching, etc. are examined. A review of existing methods for determination of the bulk etch rate and track etch rate $V_{\mathrm{t}}$ is also given. Examples of $V_{\mathrm{t}}$ functions for some detectors are presented. Some unsolved questions related to $V_{\mathrm{t}}$ and some contradictory experimental results published in the literature are also summarized in the paper.

Applications of SSNTDs for radon and progeny measurements are discussed. New designs of diffusion chambers that have appeared in the last few years are portrayed. A review of analytical and Monte Carlo methods for the calculation of the calibration factors in radon measurements is presented.

Particular attention has been given to methods of long-term passive measurements of radon progeny with SSNTDs. These measurements are rather difficult and there is not yet a widely accepted solution. One possible solution based on the LR 115 SSNTD is outlined here.

Methods for retrospective radon measurements are also described. Various applications of SSNTDs in other fields of physics and other sciences are briefly reviewed at the end of the paper.

(C) 2004 Elsevier B.V. All rights reserved.
\end{abstract}

Keywords: Nuclear tracks; Solid-state nuclear track detector; SSNTD; CR-39; LR 115

\section{Introduction}

\subsection{History of solid-state nuclear track detectors (SSNTDs)}

The science of solid-state nuclear track detectors was born in 1958 when D.A. Young discovered the first tracks in a crystal of LiF [1]. The etch pits, later called "tracks", were found in a LiF crystal which was previously placed in contact with a uranium foil, irradiated with slow neutrons and treated with a chemically aggressive solution. The thermal neutrons led to fission of the uranium nuclei and the fission fragments bombarded the LiF crystal and damaged it. The damaged regions constituted more chemically active zones than the surrounding undamaged areas. One year later, Silk and Barnes [2] reported the finding of damaged regions in mica. They used the transmission electron microscope to investigate tracks of heavy charged particles in mica. Fleischer et al. [3] conducted extensive

\footnotetext{
* Corresponding author. Tel.: +852 27887812; fax: +852 27887830.

E-mail address: peter.yu@ cityu.edu.hk (K.N. Yu).
} 
investigations of this method. They applied the method to mica and to other materials like minerals, plastics and glasses.

Since then, there was a tremendous growth in this field of science. There were many articles published in scientific journals and several books were published on these topics [4-7]. The series of conferences on nuclear tracks started by the First International Colloquium on Corpuscular Photography in 1957 in Strasbourg, France. Subsequent conferences have been held in 1958, 1960, 1962, 1964, 1966, 1970, 1972, 1976, 1979, 1981, 1983, 1985, 1988 and 1990, with the name of the conference changed to International Conference on Nuclear Photography since 1964, International Colloquium on Corpuscular Photography and Visual Solid Detectors in 1970, International Conference on Nuclear Photography and Solid-State Detectors in 1972, International Conference on Solid-State Nuclear Track Detectors since 1976, and International Conference on Particle Tracks in Solids in 1990. Since 1992, the name of the conference has been changed to International Conference on Nuclear Tracks in Solids. The last one of the series was the 21st International Conference on Nuclear Tracks in Solids held in New Delhi, India in 2002, and the coming one will be the 22nd International Conference on Nuclear Tracks in Solids to be held in Barcelona, Spain in 2004. Several thousands of papers have been reported in these conferences. The proceedings of the last five conferences can be found in [8-12].

\subsection{Basics}

Operation of the solid-state nuclear track detector is based on the fact that a heavy charged particle will cause extensive ionization of the material when it passes through a medium. For example, an alpha particle with energy of $6 \mathrm{MeV}$ creates about 150,000 of ion pars in cellulose nitrate. Since the range of a $6 \mathrm{MeV}$ alpha particle in this material is only about $40 \mu \mathrm{m}$, that means on average 3700 ion pairs are created per micrometer, or 3-4 ion pairs per nanometer. An alpha particle ionizes almost all molecules close to its path. This primary ionizing process triggers a series of new chemical processes that result in the creation of free chemical radicals and other chemical species. Along the path of the alpha particle, a zone enriched with free chemical radicals and other chemical species is then created. This damaged zone is called a latent track.

If a piece of material containing latent tracks is exposed to some chemically aggressive solution, chemical reactions would be more intensive along the latent tracks. Aqueous solutions of $\mathrm{NaOH}$ or $\mathrm{KOH}$ are the most frequently used chemical solutions in this regard. The overall effect is that the chemical solution etches the surface of the detector material, but with a faster rate in the damaged region. In this way, a "track" of the particle is formed, which may be seen under an optical microscope. This procedure is called "detector etching" or track visualization, and the effect itself is called the "track effect".

The track effect exists in many materials. It is particularly pronounced in materials with long molecules, e.g., cellulose nitrates or different polycarbonates, and such materials are the most convenient ones for application and detector manufacturing. The effect is also seen in some amorphous materials like glasses, etc. However, only dielectric materials show the track effect. In conductive materials and in semiconductors, the process of recombination occurs and the latent tracks are not stable. A comprehensive survey on the materials that show the track effect is given by Fleischer et al. [4].

One of the most commonly used nuclear track detectors is the CR-39 detector, which was discovered by Cartwright et al. [13], is based on polyallyldiglycol carbonate. Another most commonly used nuclear track material is cellulose nitrate. The most well-known detector in this group is being sold under the commercial name LR 115. Other kinds of detectors are also in use, such as the Makrofol 
detector which is based on polycarbonate. Some natural materials that show the track effect, such as apatite, mica, olivine, etc. are used for fission or fossil track studies.

As mentioned earlier, etching of irradiated conductive materials would not produce visible tracks. The condition for stable latent-track formation is sometimes expressed as a limiting value for the material resistivity. However, there is not a unique value of resistivity above which the track effect always appears. In this way, the material resistivity cannot serve as the unique criterion for track formation.

Although the track effect is relatively well known, and the technique is rather simple and straightforward, there is not a unique theory that explains track formation. The basic physical processes after the initial charged particle loses its energy are the ionization and excitation of molecules of the material. This first "physical" phase in which the initial particle delivers its energy to the atoms surrounding its path is very short in time; stopping of the particle occurs within a time of the order of picoseconds. The free electrons created in these primary interactions will slow down through a series of ionizations and excitations, and will create more and more free electrons. Some of these may go further away from the initial particle path creating the so-called delta $(\delta)$ rays. A large number of free electrons and damaged molecules are created close to the particle track.

In the second physiochemical phase, new chemical species are created by interactions of the damaged molecules. During etching, the interactions of these new chemical species with the etching solution are stronger than that with the undamaged detector material. However, it is not known which chemical species are formed after the particle passage through the material, and the nature of damage is also not entirely known. Different theories have been developed in order to understand these processes [14-17].

\subsection{Stopping power, restricted energy loss}

As described earlier, the primary process of charged-particle interaction with the detector material is ionization and excitation of the molecules in the detector. The initial charged particle loses its energy through the many interaction processes. Theoretically, it interacts through Coulomb force with charged particles (electrons and nuclei) in the material. Of course, distal interactions may be neglected and we focus on the particle interactions with atoms and molecules that are close to its path. The majority of the interactions occur with electrons and only a small number of interactions are with nuclei. Since the initial heavy charged particle (only such particles can produce tracks) is much heavier than electrons, the direction of the particle effectively does not change and the path is almost completely a straight line. This may not be true if the particle interacts with a nucleus, where a significant deviation from the initial direction may occur. However, such interactions are relatively rare. Some deviations from the straight line can happen close to the end of the particle range, when the energy of a particle becomes very low.

The particle loses its energy in many small interaction processes, so the energy loss each time is usually very small when compared to its energy. For example, ionization of one molecule in air on average needs about $32 \mathrm{eV}$, which is $10^{-5}$ to $10^{-6}$ of the particle energy (assuming that the particle energy is in the $\mathrm{MeV}$ region). As a result of these many small interaction processes, the particle will continuously slow down in the detector material. The physical quantity that describes the slowing down of charged particles in mater is the stopping power $-\mathrm{d} E / \mathrm{d} x$ (or the stopping force used by some authors), where $\mathrm{d} E$ is the energy lost in the distance $\mathrm{d} x$. Stopping power is given in $\mathrm{J} / \mathrm{m}$ or in $\mathrm{keV} / \mu \mathrm{m}$. The energy lost by a particle in the distance $\mathrm{d} x$ is the energy transferred to the material so this quantity is also called the linear energy transfer (LET). The importance of stopping power in the field of nuclear track detectors was discussed in [18]. The most recent description of particle energy loss processes is given in [19]. 
Historically, the first expression for the stopping power was given by Bohr [20]. That was a classical consideration of the particle interaction with a free electron, where the energy lost in the collision with one electron was integrated in some assumed limits of interaction and the expression for the stopping power was derived. This was modified by taking into account the quantum effects by Bethe [21], and the relativistic effects by Bloch [22], and finally the well-known Bethe-Bloch expression for the stopping power was given as:

$$
-\frac{\mathrm{d} E}{\mathrm{~d} x}=\frac{Z^{2} e^{4}}{4 \pi \varepsilon_{0}^{2} m_{0} v^{2}} N\left[\ln \frac{2 m_{0} v^{2} W_{\max }}{\bar{I}^{2}\left(1-\beta^{2}\right)}-2 \beta^{2}-\delta-U\right]
$$

where $Z$ was the charge of the incident particle, $v$ its velocity, $\beta=v / c, m_{0}$ the rest mass of the electron, $N$ the number of electrons per unit volume, $\bar{I}$ the average excitation potential of electrons in the stopping material, $W_{\max }$ the maximal value of transferred energy of electron, $\delta$ the correction for polarization of the material and $U$ takes into account non-participation of inner electrons in the collision. The stopping power given in the above equation takes into account only collisions with electrons. Events with nuclei are not considered in this formula.

There is one important drawback of this formula. It was derived using the perturbation theory and the first Born approximation. In the low-energy region, this approximation may not be valid, i.e., the function inside the logarithm may become less than 1 and then the whole expression becomes negative in the low-energy region. In addition, this formula does not take into account some effects that appear when the energy of particles falls below some limits. Slowly moving charged particles can experience the charge exchange process, viz. capture of an electron in a collision with an atom of the stopping medium, and lose it in subsequent collisions. The charge of ions is not equal to $Z$ and the effective charge $Z_{\text {eff }}$ should be introduced to describe the process. However, such a simple change of the formula is not sufficient to describe the complicated process of charge exchange.

As described earlier, when the particle loses energy in the material, part of the energy is taken by energetic electrons which can then go far away from the initial particle path and are called $\delta$ rays. This energy is spent far away from the particle path and does not take part in the formation of the particle track. For this reason, a new quantity called the restricted energy loss (REL) was introduced $(-\mathrm{d} E / \mathrm{d} x)_{E_{\mathrm{b}}}$. Only energy transfers smaller than $E_{\mathrm{b}}$ are considered for calculations of $\mathrm{d} E$. Here, for collisions with energy transfer larger than $E_{\mathrm{b}}, \delta$ electrons are assumed to be formed, which do not take place in track formation. As we will see later, there is not a unique value for $E_{\mathrm{b}}$.

The concept of REL is also used in other fields of physics. However, this quantity is not unique, and may be different for various materials. For this reason, a microdosimetric quantity called lineal energy, which does not have such shortcomings, is used.

Nowadays, some computer softwares are available for the calculation of stopping power and range of charged particles in different media. The most well-known one is the SRIM (Stopping and Range of Ions in Matter) program developed by Ziegler et al. [23].

\section{Geometry of track development}

\subsection{Geometry of track development and basic terms}

One of the challenges that have attracted significant amounts of attention was a formal description of the track development, i.e., growth of tracks. The problem is rather geometrical in nature. In addition, there are theories that describe the physical aspect of track formation. However, until now, 


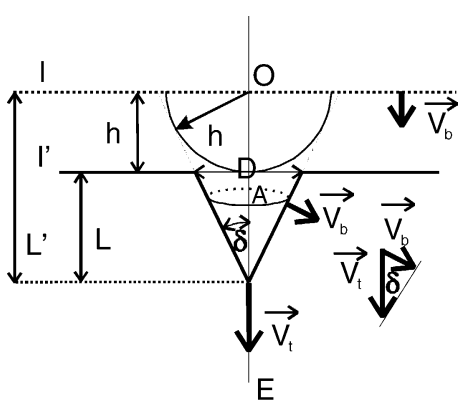

Fig. 1. Geometry of the track development. The incident angle is normal with respect to the detector surface, and $V_{\mathrm{t}}$ is constant.

there is not a single complete theory that satisfactorily explains track formation and calculates the parameters related to the tracks.

The simplest case of track development refers to that when the incident particle enters a detector under normal incidence with respect to the detector surface as shown in Fig. 1 . In this figure, $I$ is the initial detector surface, $I^{\prime}$ is the surface after the etching, $V_{\mathrm{t}}$ is the etch rate along the particle trajectory (track etch rate), $V_{\mathrm{b}}$ is the etch rate of the undamaged regions of the detector (bulk etch rate), $O$ is the entrance point and $E$ is the end point of a particle in the detector material, and $\mathrm{OE}=R$ is the particle range in the detector material. The distance between $I$ and $I^{\prime}$ is equal to $h$, i.e., the thickness of the layer removed by etching, $L^{\prime}$ is the total distance traveled by the etching solution along the particle track, and $L$ is the track depth.

In one aspect, track development is analogous to wave propagation. According to the Huygen's principle, each point in the wave front is the source of a new spherical wave. In the case of the track development, a hemisphere with a radius $h=V_{\mathrm{b}} t$ ( $t$ is the etching time) is formed around each point on the detector surface, except in the direction of the particle path where the etching progresses with the rate $V_{\mathrm{t}}$.

Track development is governed by the ratio $V=V_{\mathrm{t}} / V_{\mathrm{b}}$ and track formation is not possible if $V$ is smaller than or equal to 1 . In other words, the condition $V>1$ must be fulfilled to form a track. The angle $\delta$ in Fig. 1 is called the local developing angle.

From the similarity of triangles in Fig. 1, it can be observed that:

$$
\sin \delta=\frac{1}{V}
$$

As mentioned earlier, the etching progresses in all directions with the rate $V_{\mathrm{b}}$ except along the particle path where the etching goes with the rate $V_{\mathrm{t}}$. In three dimensions, the track is a cone with the developing angle $\delta$, which is obtained by rotation of the track wall around the particle path. The circle $A$ in Fig. 1 represents the revolution of a point on the track wall around the particle path. During etching, the track wall moves parallel to itself.

There are different phases of the track development which will be discussed later in details. The geometry of the track development has been considered by a number of authors (e.g., [24-34]).

\subsection{Geometry of track development for constant $V_{t}$}

\subsubsection{Constant $V_{t}$ and normal incidence}

Recalling the analogy between track development and wave propagation according to the Huygen's principle, track development can be visualized as something similar to the wave formation 
behind the boat moving on the surface of still water. The difference is that the wave formed behind the boat has essentially a two-dimensional appearance, while the track is formed as a three-dimensional structure. The cross-section of the post-etch surface $I^{\prime}$ (Fig. 1) and the cone is a circle with a diameter $D$, i.e., the radius of the track opening. For the sake of simplicity, the problem can be considered in two dimensions as shown in Fig. 1.

The track depth is given by:

$$
L=\left(V_{\mathrm{t}}-V_{\mathrm{b}}\right) t
$$

where $t$ is the etching time. From Fig. 1, we can see that:

$$
\tan \delta=\frac{D / 2}{L}=\frac{h}{\sqrt{L^{\prime 2}-h^{2}}}
$$

and by combining the previous equations, one can find the diameter of the track opening as:

$$
D=2 h \sqrt{\frac{V-1}{V+1}}
$$

If $V \gg 1$, from the previous equations, we have:

$$
D \cong 2 h
$$

Based on Eq. (2.5), an indirect method for bulk etch rate measurements was developed. If the track etch rate is very large, which is the case when heavy ions or fission products are used for the irradiation, the removed layer would be directly related to the track-opening diameter which is easily measurable. Since $h=V_{\mathrm{b}} t$, it is easy to find $V_{\mathrm{b}}$.

During etching, the aggressive solution progresses toward the end point $E$ of the particle trajectory. The track end is sharp and the track is fully conical. However, at the time $t_{0}$, the etchant reaches the end point $E$ of the particle path. The detector surface at time $t_{0}$ is denoted as surface 1 in Fig. 2. The etching after that moment progresses in all directions with the same rate $V_{\mathrm{b}}$, and the corresponding track becomes an "over-etched" one. A sphere is now formed around the point $E$, and the shape of the track has changed to one with a cone jointed with a sphere (surfaces 2 and 3 in Fig. 2). With prolonged etching, the spherical part is enlarged and the conical part is relatively smaller and smaller. Finally, if the etching lasts sufficiently long, the whole track will become spherical (surface 4

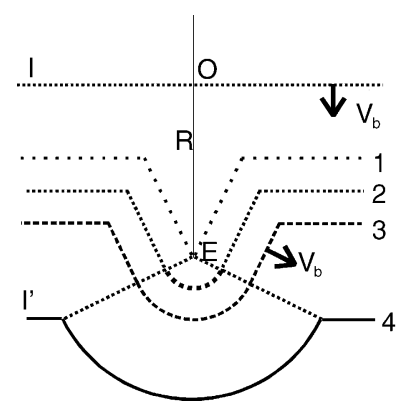

Fig. 2. Three phases in the track development. $I$ is the initial detector surface, $O$ and $E$ are the entrance and end points of the particle path, $R$ is the particle range in the detector material, and $V_{\mathrm{b}}$ is the bulk etch rate. (1) Conical track; (2 and 3) the track wall is partially conical and partially spherical; (4) the track is fully spherical. 
in Fig. 2). The contrast of a spherical track is lost and the track might be seen with difficulties or might even become invisible.

\subsubsection{Constant $V_{t}$ and oblique incidence}

In most realistic applications, the incident particles strike the detector with oblique incidence instead of normal incidence. A typical example is the irradiation of a detector by alpha particles emitted by radon and its progeny, where all incident angles are possible. Another example is irradiation by cosmic rays. It is therefore important to describe track growth for oblique incidence.

This problem was considered in detail by Somogyi and Szalay [25]. The cross-section between a track in the conical phase and the post-etching surface is an ellipse and the corresponding track opening is elliptical. The ellipse is characterized by its major axis $D$ and its minor axis $d$. These two parameters are important characteristics of a track opening for oblique incidence. If the track is overetched, the post-etching surface might cut both the elliptical and spherical parts of the track wall. In this case, the track-opening contour is a complex curve that consists of an ellipse and a circle jointed at some points. With prolonged etching, the spherical part of the track wall, and thus the circular part of the track opening, are enlarged. Finally, the track becomes totally spherical and the opening becomes completely circular.

According to Somogyi and Szalay [25], during the etching, the major axis of the track opening passes through three phases, while the minor axis develops through two phases. Analytical formulas for the major axis in the three phases $\left(D_{1}, D_{2}\right.$ and $\left.D_{3}\right)$ and those for the minor axis in the two phases $\left(d_{1}\right.$ and $d_{2}$ ) were derived and expressed in terms of removed layer $h$. The conditions for transition from one phase to another were also derived in terms of $h$. Somogyi and Szalay [25] also denoted different phases of axis development as $D_{1}, D_{2}$ and $D_{3}$ or $d_{1}$ and $d_{2}$. The different phases of track development for a particle striking the detector under oblique incidence are shown in Fig. 3. Here, the incident angle $\theta$ is measured with respect to the detector surface, and $h_{0}$ is the removed layer when the etchant reaches the end point $E$ of the particle path.

In the first phase, $D_{1}$, the opening contour is an ellipse; in the third phase, $D_{3}$, the opening is fully circular; while in the second phase, $D_{2}$, it is a transition between the two, i.e., the contour opening is a partial ellipse jointed to a partial circle. In the phase $d_{1}$, the opening is elliptical and the minor axis is the minor axis of the ellipse. In the phase $d_{2}$, the opening contour is a circle and the minor axis is the radius of the circle.

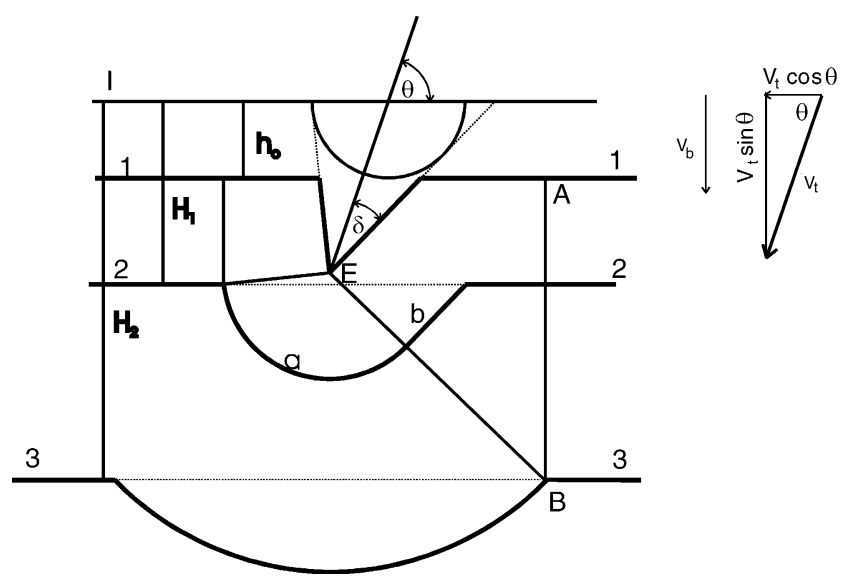

Fig. 3. Phases of track development for oblique incidence. 
The formulas for calculating the major and minor axes of the track opening, and the conditions of validation are summarized by the following equations [25]:

$$
\begin{aligned}
& d_{1}=2 h \sqrt{\frac{V \sin \theta-1}{V \sin \theta+1}} \text { for } 0 \leq h \leq h_{1}=\frac{R}{V}+R \sin \theta \\
& d_{2}=D_{3} \text { for } h_{1} \leq h \\
& D_{1}=2 h \frac{\sqrt{V^{2}-1}}{V \sin \theta+1} \text { for } 0 \leq h \leq H_{1}=\frac{R}{V}+R \frac{V \sin \theta-1}{V-\sin \theta+\cos \theta \sqrt{V^{2}-1}} \\
& D_{2}=\frac{1}{2}\left(D_{1}+D_{3}\right)+\left(R-\frac{h V}{V \sin \theta+1}\right) \cos \theta \text { for } \\
& H_{1} \leq h \leq H_{2}=\frac{R}{V}+R \frac{V \sin \theta-1}{V-\sin \theta-\cos \theta \sqrt{V^{2}-1}} \\
& D_{3}=2 \sqrt{R\left(\sin \theta-\frac{1}{V}\right)\left[2 h-R\left(\sin \theta+\frac{1}{V}\right)\right]} \text { for } h \geq H_{2}
\end{aligned}
$$

\subsection{Geometry of track development for variable $V_{t}$}

In the previous section, the model of track growth for a constant $V_{\mathrm{t}}$ developed by Somogyi and Szalay [25] has been presented. However, $V_{\mathrm{t}}$ is not constant in most of the realistic cases. The same authors developed equations for the case of a variable $V_{\mathrm{t}}$. Other authors have also developed their own models and methods to describe track growth, which will be shortly presented here. The difference in comparison to the constant $V_{\mathrm{t}}$ case is that the track wall cannot be described as a regular cone, and the track is now a semi-conical surface shown in Fig. 4 (right panel, case 2). The cross-section between the post-etching detector surface and the track is now more complex than a simple ellipse. It can be close
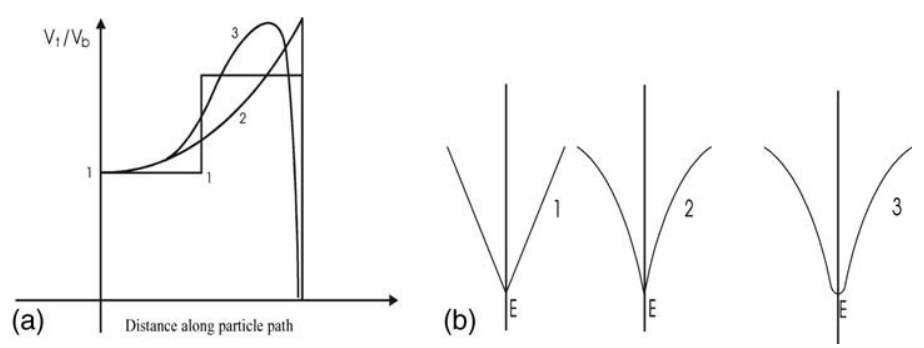

Fig. 4. (a) Variation of the $V_{\mathrm{t}}$ function along the particle path: (1) $V_{\mathrm{t}}=$ constant; (2) $V_{\mathrm{t}}$ is variable with maximum at the end of the particle path; (3) $V_{\mathrm{t}}$ is variable with the maximum before the end of particle path (this is the realistic situation). (b) Track profiles for the cases (1), (2) and (3), respectively. 
to an ellipse, but it might also be very different from an ellipse, such as some egg-like curve, etc. depending on the removed layer, range and $V_{\mathrm{t}}$.

\subsubsection{Somogyi-Szalay model for variable $V_{t}$ and some more considerations about track development}

Somogyi and Szalay [25] kept the same concepts of $D_{1}, D_{2}$ and $D_{3}$ introduced for the major axis, and $d_{1}$ and $d_{2}$ for the minor axis. The equations governing the transition from one phase to another are somewhat modified from the equations for the constant $V_{\mathrm{t}}$ case. The set of equations for the etch pit wall in two dimensions was also given in parametric form. The solutions of this set of equations require the iteration approach.

The track growth model of Somogyi and Szalay [25] inherently assumed that the track is sharp at point $E$ when the etchant reaches the end point of the particle path. Criteria for transition from one phase to another are based on that assumption. The assumption that the track is sharp at point $E$ is a consequence of the simplifying assumption about the characteristics of $V=V_{\mathrm{t}} / V_{\mathrm{b}}$, which is equivalently a simplifying assumption about the stopping power of heavy charged particles in the detector material. These are explained in Fig. 4. In Fig. 4(a), various $V_{\mathrm{t}}$ functions are presented. The curve denoted by 1 presents a constant $V_{\mathrm{t}}$. One should note that $V=1$ from the detector surface down to some depth in the detector material and becomes larger thereafter. Track formation begins when the etchant reaches that depth. The curve denoted by 2 represents a variable $V_{\mathrm{t}}$ function with the maximum at the end point of the particle trajectory, while the curve denoted by 3 represents a variable $V_{\mathrm{t}}$ function with the maximum before the end point.

In Fig. 4(b), the different track profiles at the moment when the etchant reaches point $E$ are shown. The tracks presented in Fig. 4(b) correspond to the $V_{\mathrm{t}}$ functions shown in Fig. 4(a). One should note that the track denoted as 3 in Fig. 4(b) is rounded at the moment when the etchant reaches point $E$. Such kind of behavior is caused by the existence of the Bragg peak in the stopping power curve in Fig. 4(a), i.e., the stopping power has a maximum close to the end of particle path. For example, for the CR-39 detector, the maximum is $2.2 \mu \mathrm{m}$ before the end of the path. The Somogyi and Szalay model [25] was developed for the $V_{\mathrm{t}}$ function (2) in Fig. 4(a), so the track profile is the curve labeled as 2 in Fig. 4(b). The track rounding before point $E$ has not been taken into account. The conditions derived for the transition between different phases are only approximate. In addition, track development in the over-etched phase will follow the shape of the track end at point $E$, which might not be a sphere. Therefore, it is necessary to develop a model for track growth which is independent of the transition conditions.

\subsubsection{Fromm et al. model}

This model was firstly presented by Fromm et al. [28] as well as in some subsequent publications by the same group [29]. The model is also two-dimensional and two-parametric, and the schematic diagram for the model is given in Fig. 5.

In their model, the action of etching is decomposed into two alternative steps using first a variable velocity $V_{\mathrm{t}}$ to etch the damaged ion trajectory and then a constant velocity $V_{\mathrm{b}}$ to enlarge the track outside the damaged regions of the etched path. The $V_{\mathrm{t}}$ varies with respect to the variation of energy deposition along the ion path. On the other hand, $V_{\mathrm{b}}$ is assumed to produce an isotropic etching process around the trajectory position $\lambda_{i}$, which constitutes an iterative step during the computation. At the depth $h=V_{\mathrm{b}} t$, the envelopes of all spheres with centers on the particle path constitute the etched track wall.

In the case where the track is tilted at an angle $\Phi$ with respect to the normal to the surface, the coordinates of the couples of points $\left(P_{1}, P_{2}\right)$ which constitute the track profile in a sagittal section 


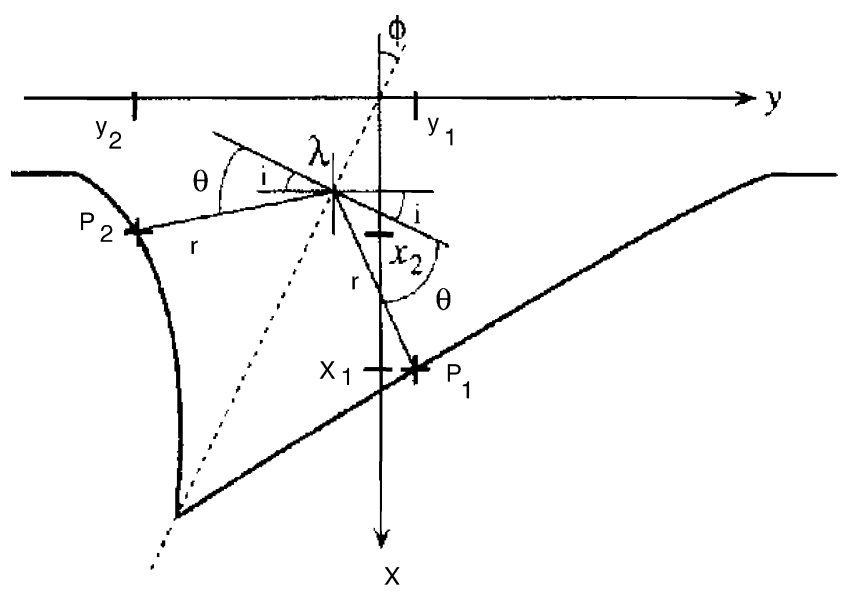

Fig. 5. Coordinates of the points that constitute the walls of an etched track in a sagittal section [28].

(see Fig. 5) are given as:

$$
\begin{aligned}
& P_{1}\left\{\begin{array}{l}
x_{1}=\lambda_{1} \cos \Phi+V_{\mathrm{b}} \tau \sin (\theta+\Phi) \\
y_{1}=\lambda_{1} \sin \Phi+V_{\mathrm{b}} \tau \cos (\theta+\Phi)
\end{array}\right. \\
& P_{2}\left\{\begin{array}{l}
x_{1}=\lambda_{i} \cos \Phi+V_{\mathrm{b}} \tau \sin (\theta-\Phi) \\
y_{1}=\lambda_{i} \sin \Phi+V_{\mathrm{b}} \tau \cos (\theta-\Phi)
\end{array}\right.
\end{aligned}
$$

with

$$
\tau=t-t_{i}, \quad t_{i}=\int_{0}^{\lambda_{i}} \frac{\mathrm{d} \lambda}{V_{\mathrm{t}}}
$$

The coordinates (see Fig. 6) of the couples of points $\left(Q_{1}, Q_{2}\right)$ which constitute the perimeter of the opening of a track are:

$$
Q_{1}, Q_{2} \mid \begin{aligned}
& x=V_{\mathrm{b}} t \\
& y=\frac{1}{\sin \phi}\left(V_{\mathrm{b}} t \cos \Phi-\lambda_{i}-V_{\mathrm{b}} \tau \sin \theta\right) \\
& z= \pm \sqrt{V_{\mathrm{b}}^{2} \tau^{2}-\left(V_{\mathrm{b}} \tau-\lambda_{i} \cos \Phi\right)^{2}-\left(y-\lambda_{i} \sin \Phi\right)^{2}}
\end{aligned}
$$

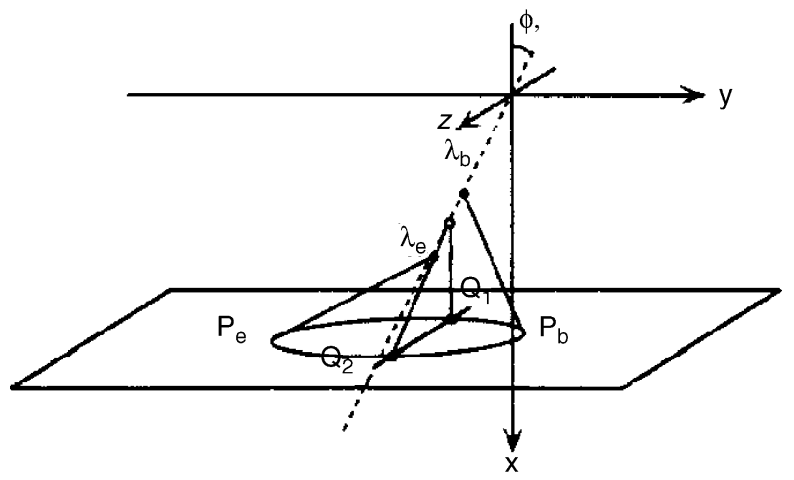

Fig. 6. Construction of the track opening [28]. 
Only the part of the particle track between $\lambda_{\mathrm{b}}$ and $\lambda_{\mathrm{e}}$ generates the contour of the track opening. The boundaries are obtained by solving the two limit equations:

$$
\begin{aligned}
& P_{\mathrm{b}}(x)=V_{\mathrm{b}} t \Leftrightarrow \lambda_{\mathrm{b}} \cos \Phi+V_{\mathrm{b}} \tau \sin (\theta+\Phi)-V_{\mathrm{b}} t=0 \\
& P_{\mathrm{e}}(x)=V_{\mathrm{b}} t \Leftrightarrow \lambda_{\mathrm{e}} \cos \Phi+V_{\mathrm{b}} \tau \sin (\theta-\Phi)-V_{\mathrm{b}} t=0
\end{aligned}
$$

where the subscripts ' $b$ ' and 'e' refer to the beginning and the end, respectively.

\subsubsection{Fews and Henshaw model}

This model was given by Fews and Henshaw [34]. The analysis provided there was based on the alpha-particle track structure in the CR-39 detector. They emphasized that a track could be analyzed by looking at five parameters, namely, the track depth $Z$, the major axis $D\left(M_{j}\right.$ in their nomenclature), the minor axis $d$ (also $M_{i}$ in their nomenclature), the overall track length $X$ and the diameter $m$ of the etched-out end (which was equal to zero in the case of a non-etched-out track).

The characteristics of the alpha-particle track and the relevant parameters used are summarized in Fig. 7. The general case of etching for an arbitrary amount beyond the end of the particle range is shown.

For the section shown in Fig. 7, the particle has a residual range $\mathrm{PR}=r$ at point $P$. The relation between the angle $\Phi$ (not the same angle as in the model of Fromm et al. [28]) and the distance $a^{\prime}=\mathrm{Q}^{\prime} \mathrm{R}$ was derived. The set of equation was given as:

$$
a^{\prime 2}=\left(\mathrm{QP}+\frac{m}{2}\right)\left[1-\left(\frac{V_{\mathrm{b}}}{V_{\mathrm{t}}}\right)^{2}\right]+\left(r-\frac{m}{2} \frac{\mathrm{PS}}{\mathrm{QP}}-\mathrm{PS}\right)^{2}
$$

where

$$
\begin{aligned}
& \mathrm{QP}=\int_{0}^{r} \frac{V_{\mathrm{b}}}{V_{\mathrm{t}}(r)} \mathrm{d} r \quad \text { and } \quad \mathrm{PS}=\frac{V_{\mathrm{b}}}{V_{\mathrm{t}}(r)} \mathrm{QP} \\
& \Phi^{\prime}=\sin ^{-1}\left[\frac{\mathrm{QP}+(m / 2)}{a^{\prime}} \sqrt{1-\left(\frac{V_{\mathrm{b}}}{V_{\mathrm{t}}}\right)^{2}}\right]
\end{aligned}
$$

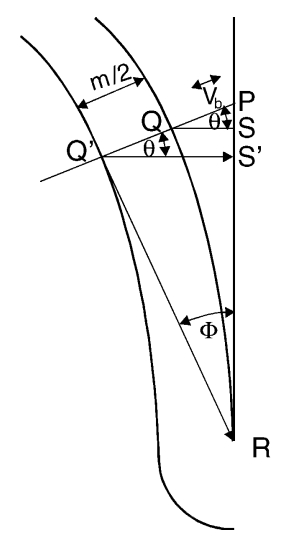

Fig. 7. Part of the vertical section of an alpha-particle track etched to an arbitrary amount beyond its range and the relevant parameters [34]. $R$ is the end of particle trajectory. The particle has a residual range PR $=r$ at point $P$. 


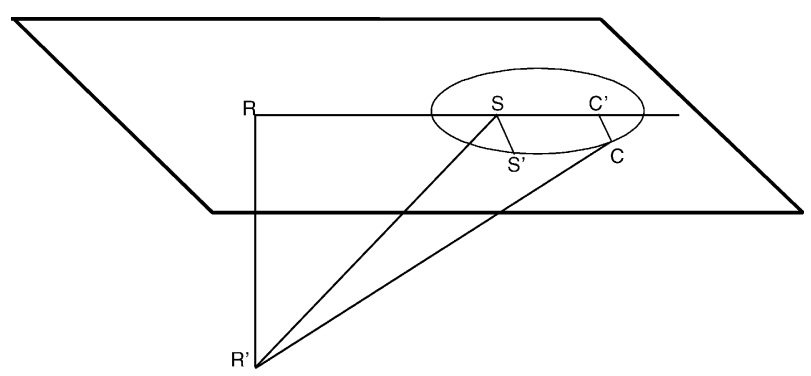

Fig. 8. An element of the post-etched plastic surface illustrating the relevant parameters used to describe the opening of the etched track [34].

The track opening given in Fig. 8 is described by the following equations:

$$
\begin{aligned}
& \mathrm{R}^{\prime} \mathrm{C}=\sqrt{a^{\prime 2}-(L \sin \delta-\Delta)^{2}} \\
& \varepsilon=\cos ^{-1}\left[\frac{\left(\mathrm{R}^{\prime} \mathrm{C}\right)^{2}+\left(\mathrm{R}^{\prime} \mathrm{S}\right)^{2}-(\mathrm{SC})^{2}}{2\left(\mathrm{R}^{\prime} \mathrm{S}\right)\left(\mathrm{R}^{\prime} \mathrm{C}\right)}\right] \\
& a_{\mathrm{s}}^{\prime}=\sqrt{\left(L-\frac{\Delta}{\sin \delta}\right)^{2}+\frac{M_{\mathrm{p}}^{2}}{4}}
\end{aligned}
$$

where $\Delta=V_{\mathrm{b}} \tau, a_{\mathrm{s}}^{\prime}=\mathrm{RS}^{\prime}, \varepsilon=\mathrm{SR}^{\prime} \mathrm{C}, \mathrm{RS}=L-\Delta / \sin \delta$ and $\delta$ is particle dip angle with respect to the detector surface, $C$ is a general point on the track opening and the points $R^{\prime}, C, S, S^{\prime}$ and $a^{\prime}$ are described in Fig. 8.

\subsubsection{Nikezic and Yu model}

The model was presented by Nikezic and $\mathrm{Yu}$ [33] and was based on the assumption of known equations for the track wall in the two-dimensional case. The equations can be given in the form of a table or in some other forms. The equation of a track wall for normal incidence was derived in the following way. Referring to Fig. 9, a point $A$ on the track wall with coordinates $(x, y)$ was formed from the point $x_{0}$ on the particle track. From the point $(0,0)$, the etching travels with the track etch rate $V_{\mathrm{t}}$ along the $x$-axis (which is the particle trajectory) and reaches the point $x_{0}$ at time $t_{0}$. From $x_{0}$, the etching progresses to point $A$ with the bulk etch rate $V_{\mathrm{b}}$. The angle $\delta=\delta\left(x_{0}\right)$ is the angle between $V_{\mathrm{t}}$ and $V_{\mathrm{b}}$ at point $x_{0}$ as shown in Fig. 9, and could be found as:

$$
\delta\left(x_{0}\right)=\sin ^{-1}\left(\frac{1}{V\left(x_{0}\right)}\right) \text { where } V\left(x_{0}\right)=\frac{V_{\mathrm{t}}\left(x_{0}\right)}{V_{\mathrm{b}}}
$$

From geometrical considerations, it is clear that:

$$
y^{\prime}(x)=-\tan \delta\left(x_{0}\right)=-\frac{1}{\sqrt{V^{2}\left(x_{0}\right)-1}}
$$

This equation cannot be used as the track wall equation because the expression on the right depends on $x_{0}$ while the expression on the left depends on $x$. Noting that $x_{0}=x-\Delta x$, we have:

$$
\Delta x=y(x) \tan \delta\left(x_{0}\right)=-y(x) y^{\prime}(x)
$$




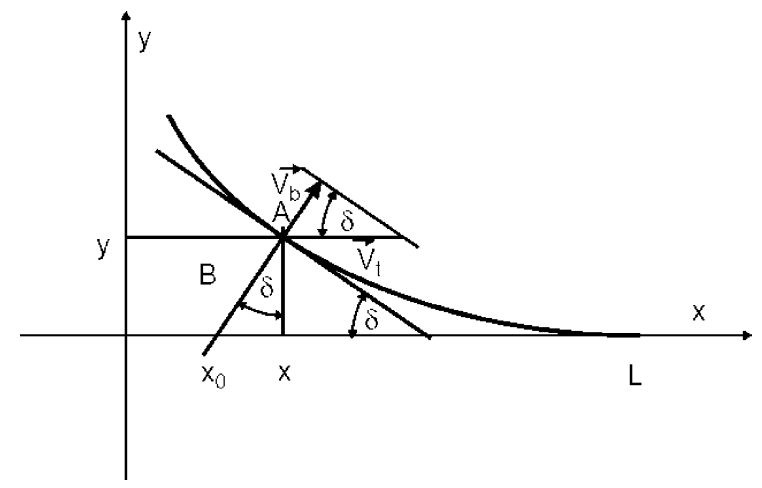

Fig. 9. Geometry of the track wall in two dimensions [33]. The particle path is along the $x$-axis.

so one can obtain:

$$
x_{0}=x+y(x) y^{\prime}(x)
$$

and

$$
y^{\prime}=-\frac{1}{\sqrt{V^{2}\left(x+y y^{\prime}\right)-1}}
$$

This is the equation of the track wall in the differential form with both sides depending only on $x$. Unfortunately, this equation cannot be solved analytically. If the angle $\delta$ is small or if $V(x)$ is a slowly varying function (which is usually the case in many applications), $y y^{\prime}$ in the denominator of Eq. (2.27) can be neglected and the approximate equation of the track wall becomes:

$$
y=\int_{x}^{L} \frac{\mathrm{d} x}{\sqrt{V^{2}(x)-1}}
$$

This approximate equation was previously used by Nikezic and Kostic [31,32]. Comparison between Eq. (2.28) and the model originally developed by the German group was presented in [35]. It has been shown that the difference is small, and that the approximate formula in Eq. (2.28) can be used for a small removed layer.

The coordinates $(x, y)$ of point $A$ can be calculated by:

$$
y=B \cos \delta\left(x_{0}\right)
$$

and

$$
x=x_{0}+B \sin \delta\left(x_{0}\right)
$$

where

$$
B=V_{\mathrm{b}}\left(T-t_{0}\right)
$$

and $T$ is the total etching time. By using Eqs. (2.29a)-(2.29c), the coordinates of the points on the track wall can be generated. A best fit of points in the wall will give:

$$
y \approx F(x, L)
$$

as the equation of the wall, where $L$ is the distance penetrated by the etching solution (see Fig. 9). However, information about $V_{\mathrm{t}}(x)$ is lost in this way. Another possibility is to solve Eq. (2.27) numerically, but this might be more complicated. 
The equation of the track wall in the conical phase in three dimensions for normal incidence can be written as:

$$
\sqrt{x^{2}+y^{2}}=F(z, L)
$$

where the $z$-axis is along the particle trajectory, and $(x, y)$ are coordinates of the points in the track wall. The track opening is circular in shape when incidence is normal, but some egg-like shape or dropletlike shape when the incidence is oblique. The contour equation for the opening is given by:

$$
\sqrt{x^{\prime \prime 2}+y^{\prime \prime 2} \sin ^{2} \theta}=F\left(y^{\prime \prime} \cos \theta+\frac{h}{\sin \theta}, L\right)
$$

where $\left(x^{\prime \prime}, y^{\prime \prime}\right)$ are coordinates on the contour of the track opening, $\theta$ is the incident angle with respect to the detector surface and $h$ is the total removed layer.

2.3.4.1. Over-etched tracks: normal incidence. The schematic sketch of an over-etched track in two dimensions is shown in Fig. 10. After a certain time of etching, the etchant will reach the end point $E$ of the particle range. At that time, the wall of the track is formed and denoted by the number 1 in Fig. 10. Point $A$ with coordinates $(z, y)$ is contained in the track wall. The angle between the tangent $t$ on the wall at point $A$ and the $z$-axis is the local development angle $\delta$. Further etching will progress in all directions with the same rate $V_{\mathrm{b}}$, including the surroundings of point $E$. As was pointed out earlier, the track profile around point $E$ in the over-etching phase will reflect the track profile when the etching solution reaches point $E$. It can be characterized by two processes only for easier treatment, namely, the displacement $d$ of the wall from position 1 to position 2, and formation of a sphere around point $E$ with the same radius $d$. Point $A$ moves normally onto the tangent $t$ for a distance $d$ to point $A^{\prime}$ with coordinates $\left(z^{\prime}, y^{\prime}\right)$. The points in the rounded part of the track are also "moved" normally onto the corresponding tangents.

From geometrical considerations, the relationship between the coordinates $(z, y)$ and $\left(z^{\prime}, y^{\prime}\right)$ are given by:

$$
z^{\prime}=z+d \sin \delta \quad \text { and } \quad y^{\prime}=y+d \cos \delta
$$

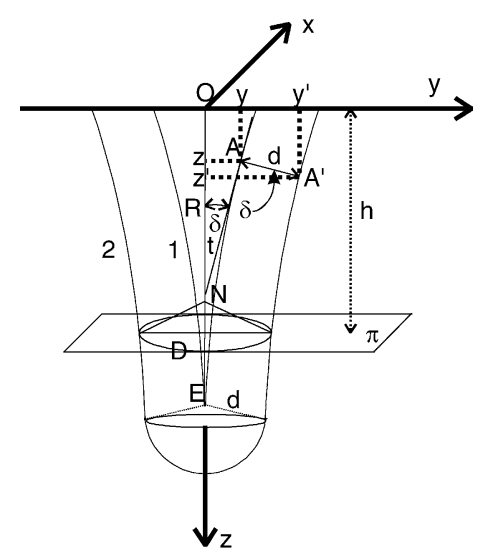

Fig. 10. An over-etched track. $O$ and $E$ are entrance and end points of a particle; $R=\mathrm{OE}$ is the particle range. Line 1: wall position at the moment when the etching solution reaches point $E$; $d$ : the removed layer during over-etching; line 2 : the new wall position; $t$ : tangent on the wall at point $A$ and $\delta$ is the local development angle of points $A$ and $A^{\prime}$. 
By using Eq. (2.29d) with $L=R$ and combining with Eq. (2.32), one can obtain the equation for the semi-conical part (in the over-etched phase) in two dimensions as:

$$
y^{\prime}=F(z, R)+d \cos \delta
$$

or

$$
y^{\prime}=F\left(z^{\prime}-d \sin \delta, R\right)+d \cos \delta
$$

One can now replace $y$ ' with $y$ and $z$ ' with $z$ to get the equation for the track wall in two dimensions as:

$$
y=F(z-d \sin \delta, R)+d \cos \delta
$$

This is the equation of the track wall in two dimensions at the moment when the etchant reaches the end point of the particle trajectory. The equation of the track wall in three dimensions is given analogously to Eq. (2.30) as:

$$
\sqrt{x^{2}+y^{2}}=F(z-d \sin \delta, R)+d \cos \delta
$$

The detector surface after etching is represented by the plane $\pi$ in Fig. 10. The plane $\pi$ is normal to the $z$-axis which represents the particle trajectory. The thickness of the removed layer is denoted by $h$ (see Fig. 10). If $z=h$ is substituted into Eq. (2.35), the equation of a circle in the plane $\pi$ is obtained as:

$$
\sqrt{x^{2}+y^{2}}=F(h-d \sin \delta, R)+d \cos \delta=D^{\prime}
$$

where $D^{\prime}$ is radius of the circle and $(x, y)$ are the coordinates of points on the circle. The angle $\delta$ in Eqs. (2.35) and (2.36) is the local developing angle at point $N$ (Fig. 10). All points of the track-opening contour were developed from the same point $N$ on the particle path under the same developing angle $\delta$. This remark can be generalized to all points with the same $z$ and same developing angle $\delta$. The diameter, $D=2 D^{\prime}$, of the circular track opening is found as:

$$
D=2 D^{\prime}=2[F(h-d \sin \delta, R)]+d \cos \delta
$$

2.3.4.2. Oblique incidence. In this part, the oblique incidence is considered. Apparently, this situation is more complicated than the previous one. However, if two transformations of the coordinate system are applied, the problem will be simplified significantly.

Semi-elliptical openings are found when the detector surface after etching does not cross the rounded part formed around point $E$. Such kind of tracks will be considered in the following. The geometry used for considering a semi-elliptical track opening is presented in Fig. 11. The nomenclature is the same as those in previous figures. A new parameter is the angle $\theta$ which is the incident angle with respect to the detector surface. Around the end point $E$ of the particle trajectory, a rounded part with radius $d$ is formed which is joined with the semi-conical part of the track wall. The plane $\pi_{1}$ represents the detector surface after etching, and $h$ is the thickness of the removed layer. The track is "cut" by the plane $\pi_{1}$ under the angle $\theta$ with respect to the particle direction ( $z$-axis). The first step is a translation of the coordinate system $(x, y, z)$ from point $O$ to point $O^{\prime}$ with coordinates $O^{\prime}\left(0,0, z_{0}\right)$ where $z_{0}=h / \sin \theta$. The newly obtained system $\left(x^{\prime}, y^{\prime}, z^{\prime}\right)$ is related to the original one through the equations:

$$
x^{\prime}=x, \quad y^{\prime}=y \quad \text { and } \quad z^{\prime}=z-z_{0}
$$

and

$$
z=z^{\prime}+z_{0}
$$




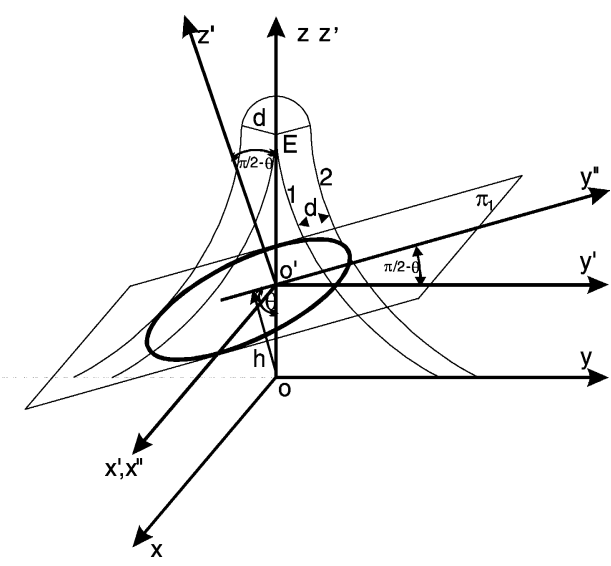

Fig. 11. Track opening in the semi-elliptical phase.

Eq. (2.35) for the track wall in the new coordinate system becomes:

$$
\sqrt{x^{\prime 2}+y^{\prime 2}}=F\left(z^{\prime}+z_{0}-d \sin \delta, R\right)+d \cos \delta
$$

The second step is a rotation of the $\left(x^{\prime}, y^{\prime}, z^{\prime}\right)$ coordinate system through an angle $(\pi / 2-\theta)$ around the $x^{\prime}$-axis. The newly formed coordinate system $\left(x^{\prime \prime}, y^{\prime \prime}, z^{\prime \prime}\right)$ system is related to the $\left(x^{\prime}, y^{\prime}, z^{\prime}\right)$ system through the equations:

$$
y^{\prime}=y^{\prime \prime} \sin \theta-z^{\prime \prime} \cos \theta \text { and } z^{\prime}=y^{\prime \prime} \cos \theta+z^{\prime \prime} \sin \theta
$$

Eq. (2.35) for the track wall in the $\left(x^{\prime \prime}, y^{\prime \prime}, z^{\prime \prime}\right)$ system is now:

$$
\sqrt{x^{\prime \prime} 2+\left(y^{\prime \prime} \sin \theta-z^{\prime \prime} \cos \theta\right)^{2}}=F\left(z^{\prime \prime} \sin \theta+y^{\prime \prime} \cos \theta+z_{0}-d \sin \delta, R\right)+d \cos \delta
$$

The surface of the detector after etching is given as $z^{\prime \prime}=0$. By substituting $z^{\prime \prime}=0$ into Eq. (2.42), the intersection between the track wall and the new detector surface described by $z^{\prime \prime}=0$ is given as:

$$
\sqrt{x^{\prime \prime 2}+y^{\prime \prime 2} \sin ^{2} \theta}=F\left(y^{\prime \prime} \cos \theta+z_{0}-d \sin \delta, R\right)+d \cos \delta
$$

Here, $x^{\prime \prime}$ and $y^{\prime \prime}$ are the coordinate axes along the plane $\pi_{1}$ (both belonging to the plane $\pi_{1}$ ) and $z^{\prime \prime}$ is normal to $\pi_{1}$. In this case, $y^{\prime \prime}$ is extended along the major axis of the track and $x^{\prime \prime}$ is normal to it. This is the equation for the contour line of the track opening in the semi-elliptical phase, where the track is rounded but has not yet passed the spherical shape. The angle $\delta$, which appears in Eq. (2.43) implicitly and varies along the contour line, makes calculations difficult. However, calculation of the contour line is facilitated by the fact that all points with the same value of $z$ have the same developing angle $\delta$ (as emphasized earlier).

The post-etching surface may cut the track partially in the rounded area and partially in the semiconical part. The obtained intersection, which is the contour of the track opening, is a complicated curve. Furthermore, the intersection may be completely across the rounded part so that the opening contour is circular. These two cases are not considered here in detail.

2.3.4.3. Major and minor axis. The track length, i.e., the major axis, can be found from Eq. (2.43) when $x^{\prime \prime}=0$. Here, the coordinates $y_{1}$ and $y_{2}$ where the contour line crosses the $y^{\prime \prime}$-axis are found as:

$$
y_{1,2} \sin \theta=\left[F\left(y_{1,2} \cos \theta+z_{0}-d \sin \delta, R\right)+d \cos \delta\right]
$$


Note that the unknown variables $y_{1}$ and $y_{2}$ are on both sides of Eq. (2.44) and iterations are needed to solve the equation. The length $D$ of the track opening is then equal to:

$$
D=\left|y_{1}\right|+\left|y_{2}\right|
$$

The track width, i.e., the minor axis, cannot be found by taking $y^{\prime \prime}=0$ because the center of the opening is shifted along the $y^{\prime \prime}$-axis. In this case, the maximum of the function given in Eq. (2.43) should be determined by locating:

$$
\left(\frac{\mathrm{d} x^{\prime \prime}}{\mathrm{d} y^{\prime \prime}}\right)_{y_{\max }}=0
$$

where $y_{\max }$ is the value of $y^{\prime \prime}$ when $x^{\prime \prime}$ has a maximum. Then, $y_{\max }$ should be substituted into Eq. (2.43) to find the maximum value $x_{\max }$. The track width (minor axis of the track opening) is given by $d=$ $2 x_{\max }$. Such procedures are rather complicated and impractical because the angle $\delta$ also depends on the coordinate $y$. A better approach is to perform calculations of $x^{\prime \prime}$ from Eq. (2.43) and to determine the maximal value of $x^{\prime \prime}$ by systematically changing values of $y^{\prime \prime}$ from $y_{1}$ to $y_{2}$.

\subsection{Comparison between different models of the track growth}

Here, we compared four different models of the track growth given by Somogyi and Szalay [25], Fews and Henshaw [34], Fromm et al. [28] and Nikezic and Yu [33]. All the models have enabled calculations of the major axis of the track opening, which is the quantity used for comparison here. Separate computer programs have been prepared for each of the models mentioned earlier, with reference to their original works. Some elements of the models are given earlier, and more details may be found in the cited references. The computer programs for the Fews-Henshaw model [34] and the Fromm et al. model [28] were developed by D. Kostic (Faculty of Science, Kragujevac, Serbia and Monte Negro). The program for the Somogyi-Szalay model was written by D. Nikezic (some descriptions of the program is given in [36]). Finally, the code for the Nikezic-Yu model was developed by the authors and can be downloaded from the web page [37].

Comparisons have been made for the CR-39 detector. The input parameters $V_{\mathrm{t}}$ and $V_{\mathrm{b}}$ given below are the same for all models. The bulk etch rate was taken as $V_{\mathrm{b}}=1.37 \mu \mathrm{m} / \mathrm{h}$. The variable track etch rate $V_{\mathrm{t}}$ was adopted from [38] as:

$$
V=1+\left(11.45 \mathrm{e}^{-0339 R^{\prime}}+4 \mathrm{e}^{-0.044 R^{\prime}}\right)\left(1-\mathrm{e}^{-0.58 R^{\prime}}\right)
$$

\subsubsection{Results of comparison}

Computations of the major axis have been performed for incident angles from $20^{\circ}$ to $90^{\circ}$ with steps of $10^{\circ}$, and for four different etching durations, namely, 3.75, 7.5, 11 and $15 \mathrm{~h}$. Since $V_{\mathrm{b}}=$ $1.37 \mu \mathrm{m} / \mathrm{h}$ is adopted, the removed layers used in calculations are 5.14, 10.275, 15.07 and $20.55 \mu \mathrm{m}$. These removed layers are denoted as (1), (2), (3) and (4) in the following text and figures. Such choices of the incident parameters and etching conditions enable comparisons of the major axis in all possible phases of the track and opening development.

The results are given in Fig. 12(a)-(h). The same nomenclature is applied in all figures: open circles for the Fromm et al. model [28], plusses for the Fews-Henshaw model [34], crosses for the Somogyi-Szalay model [25] and finally solid lines for the Nikezic-Yu model [33]. The etching conditions are given in brackets on the right-hand side of the graphs.

Fig. 12(a) gives the results for the incident angle of $90^{\circ}$. The major axis of the track opening is given as a function of the incident energy. The removed layer is used as a parameter indicated by the 

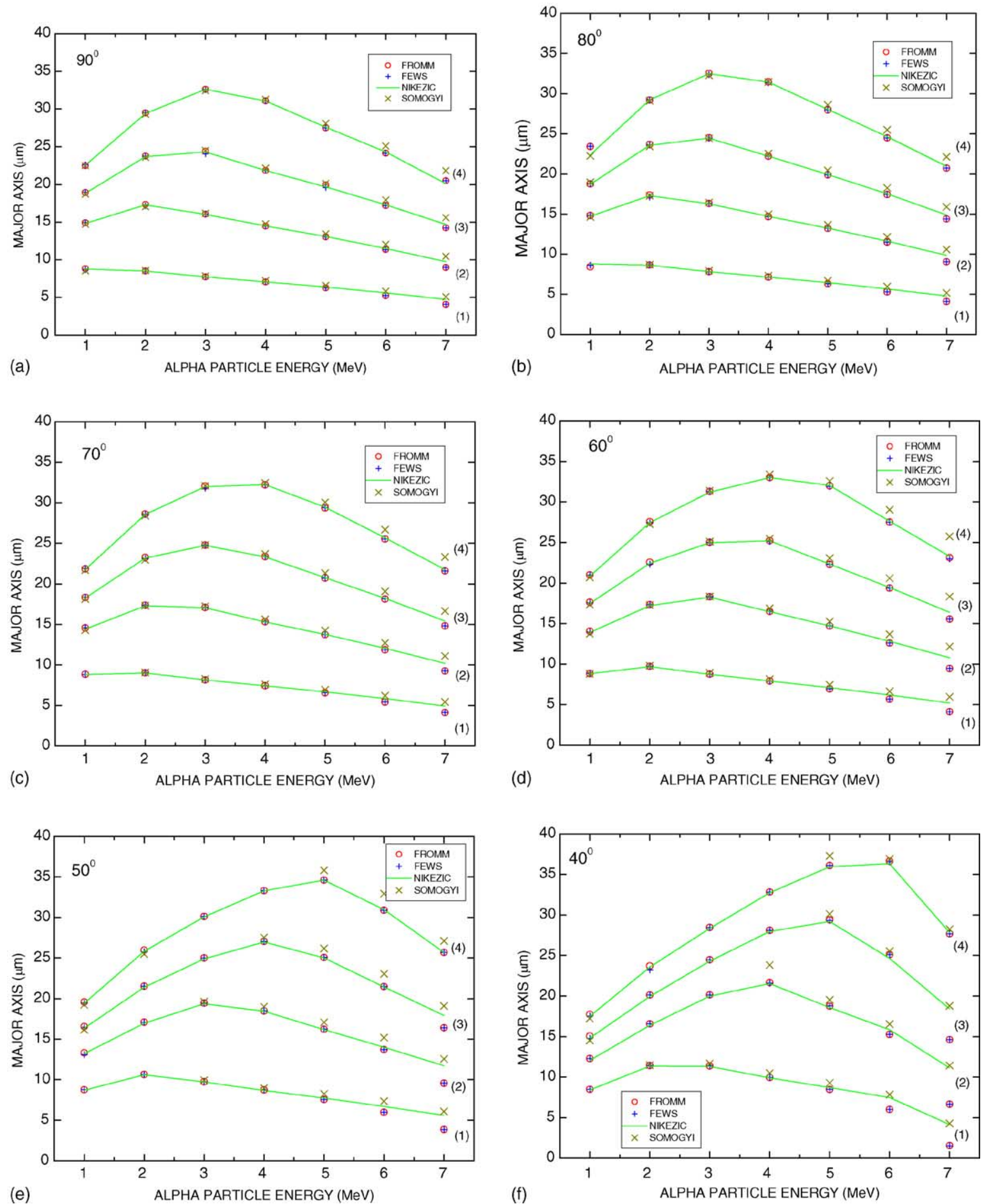

Fig. 12. Major axis calculated by four different models. Removed layers = (1) $5.14 \mu \mathrm{m}$, (2) $10.275 \mu \mathrm{m}$, (3) $15.07 \mu \mathrm{m}$ and (4) $20.55 \mu \mathrm{m}$. Incident angle: (a) $90^{\circ}$; (b) $80^{\circ}$; (c) $70^{\circ}$; (d) $60^{\circ}$; (e) $50^{\circ}$; (f) $40^{\circ}$; (g) $30^{\circ}$; (h) $20^{\circ}$. 

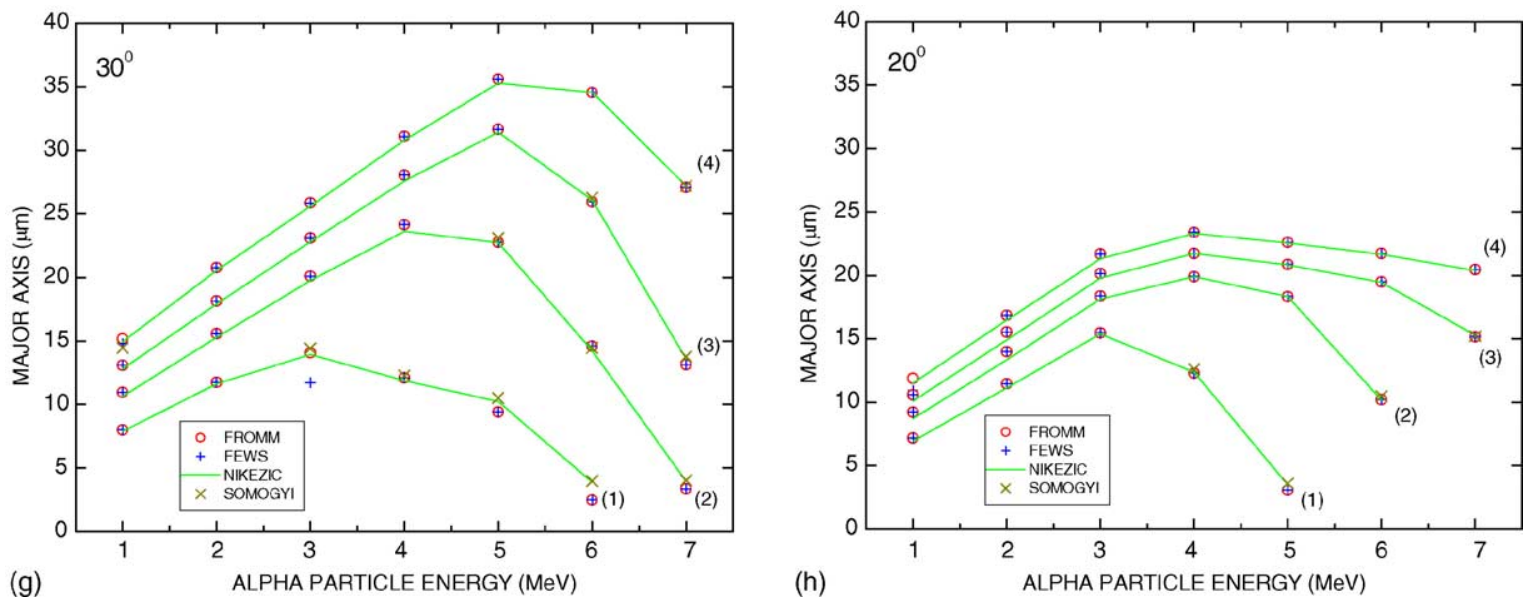

Fig. 12. (Continued).

numbers from (1) to (4) on the right-hand side of the graphs. One can see good agreement among the different models. Agreement is particularly good for the smaller incident energies. The Fews-Henshaw model [34] and the Fromm et al. model [28] are very close for all considered incident energies, and the difference is between 0.5 and 1\%. The Somogyi-Szalay model [25] agrees very well with other models, but deviates slightly for larger energies. The largest difference occurs at the largest examined energy of $7 \mathrm{MeV}$ and amounts up to $1.5 \mu \mathrm{m}$, which is $7-20 \%$ larger in comparison to the Fews-Henshaw model [34]. The Nikezic-Yu model is between the Fews-Henshaw model [34] and the Fromm et al. model [28] on one side and the Somogyi-Szalay model [25] on another side.

The results for the incident angle of $80^{\circ}$ are given in Fig. 12(b). There are no particularly new observations here when compared to Fig. 12(a). The difference, up to $1.5 \mu \mathrm{m}(8-24 \%)$, between the Somogyi-Szalay model [25] and the Fews-Henshaw model [34] is again largest at $7 \mathrm{MeV}$. The Nikezic-Yu model [33] is also between those two. For smaller energies, all models converge and the differences become smaller.

With decreasing incident angles (see Fig. 12(c)-(e)), the discrepancies among the models increase, but not significantly. The worst agreement occurs at the incident angle of $40^{\circ}$ (Fig. 12(f)) for the incident energy of $7 \mathrm{MeV}$ and etching condition (1). For these parameters, the Somogyi-Szalay model [25] gives $D=4.27 \mu \mathrm{m}$ while the Fews-Henshaw model [34] and the Fromm et al. model [28] give values about three times smaller as $D=1.55 \mu \mathrm{m}$, and the Nikezic-Yu model [33] gives $D=4.14 \mu \mathrm{m}$ which is closer to that from the Somogyi-Szalay model [25]. When increasing the removed layer corresponding to the etching conditions from (1) to (4), the results for $7 \mathrm{MeV}$ and $40^{\circ}$ converge again and almost overlap for the etching condition (4). In the lower-energy region, the agreement is also better at $40^{\circ}$.

At smaller incident angles of $30^{\circ}$ and $20^{\circ}$ (Fig. $12(\mathrm{~g})$ and $(\mathrm{h})$ ), the agreement among the models is again very good. The data are not given for all energies here because of no track formation in some cases. The largest difference occurs for $20^{\circ}, E=5 \mathrm{MeV}$ and etching condition (1), where the SomogyiSzalay model [25] gives $3.59 \mu \mathrm{m}$, the Fews-Henshaw model [34] and Fromm et al. model [28] give $3.04 \mu \mathrm{m}$, and the Nikezic-Yu model [33] gives $3.47 \mu \mathrm{m}$ for the major axis. In all other cases at $20^{\circ}$ and $30^{\circ}$, the agreement is much better.

Comparisons among the four models of track growth performed here for the major axis have shown small differences. Two of the models, by Fews and Henshaw [34] and Fromm et al. [28], agree 
to within less than $1 \%$ and the data overlap in graphs for the entire examined ranges of energies, angles and removed layers. The Somogyi-Szalay model [25] gives consistently larger values for the major axis on larger incident energies, while the Nikezic-Yu model [33] is consistently situated between the two extreme cases.

In conclusion, the examined models give practically the same values for the major axis of the track opening for wide ranges of incident angles and incident energies of alpha particles, and of removed layers.

\section{Bulk etch rate and track etch rate}

Two main parameters that govern the track formation are the bulk etch rate $V_{\mathrm{b}}$ and the track etch rate $V_{\mathrm{t}}$. These two important variables were introduced by Fleischer et al. [4].

\subsection{Bulk etch rate $V_{b}$}

The bulk etch rate $V_{\mathrm{b}}$ is the rate of removing of the undamaged surface of the detector. Due to the chemical reaction between the etching solution (etchant) and the detector material, some molecules of the detectors are removed. The final effect is the removal of the material from the detector surface. During etching, the material is removed layer by layer and the thickness of the detector becomes smaller and smaller.

\subsubsection{Indirect determination of $V_{b}$}

The bulk etch rate has been the subject of many measurements in the past, and it is probably the most frequently measured quantity concerning track detectors. It has been determined in a variety of materials, irradiated by different ions and etched under various etching conditions. There are several methods for the determination of $V_{\mathrm{b}}$, which can be broadly categorized into indirect and direct methods.

\subsubsection{Determination of $V_{b}$ from the diameter of tracks of fission fragments}

This is one of the oldest and most frequently used methods for determination of $V_{\mathrm{b}}$. Fission fragments are easily obtained from a ${ }^{252} \mathrm{Cf}$ radioactive source. Since the ranges of fission products are relatively small, the track etch rates can be considered constant during etching. Under this condition, the following simple approach can be applied. When the incidence is normal and $V_{\mathrm{t}}=$ constant, the diameter of the track opening is given by:

$$
D=2 h \sqrt{\frac{V-1}{V+1}}
$$

where $V=\left(V_{\mathrm{t}} / V_{\mathrm{b}}\right)$, and $h$ is the thickness of the removed layer during etching. If $V \gg 1$, the previous equation becomes:

$$
D \cong 2 h=2 V_{\mathrm{b}} t
$$

where $t$ is the etching time. Indirect methods for determination of $V_{\mathrm{b}}$ have thus been enabled by Eq. (3.2). The detector is first irradiated by heavy charged particles, such as fission products for which the condition $V=$ constant is effectively satisfied. After the detector is etched for some time $t$, the diameter of the track opening is conveniently measured using an optical 
microscope and $V_{\mathrm{b}}$ is determined from Eq. (3.2). This remains the most widely used method for determination of $V_{\mathrm{b}}$.

\subsubsection{Determination of $V_{b}$ from the change in detector mass}

Another method is based on measurements of the detector mass before and after etching. Based on the mass difference and the known density of the detector, it is possible to determine the thickness of the removed layer and in turn $V_{\mathrm{b}}$. This method is sometimes called the "gravimetric" method [39] and it is based on Eq. (3.3):

$$
V_{\mathrm{b}}=\frac{\Delta m}{2 A \rho t}
$$

where $\Delta m$ is mass difference, $A$ the etched surface area, $\rho$ the density of the detector and $t$ is the etching time. However, this method is limited by the accuracy of mass measurements.

\subsubsection{Determination of $V_{b}$ from spectroscopy methods}

Yip et al. [40] proposed a method to measure the thickness of the active layer of LR 115 SSNTDs based on the absorption of fluorescence X-ray photons by the active layer. A reference silver nitrate pellet is placed beneath the LR 115 detector, and a linear relationship was found between the fluorescence X-ray intensity and the thickness of the removed layer. However, there is a risk that X-ray radiation affects the track and bulk etching velocities. For example, Clark and Stephenson [41] and Fowler et al. [42] have shown X-ray degradation of cellulose nitrates. Ng et al. [43] proposed another spectroscopic method by using Fourier transform infrared (FTIR) spectroscopy, with the wave number at $1598 \mathrm{~cm}^{-1}$ corresponding to the $\mathrm{O}-\mathrm{NO}_{2}$ bond. They found an exponential decay relationship between the infrared transmittance at the wave number at $1598 \mathrm{~cm}^{-1}$ and the thickness of the active layer for LR 115 detector.

\subsubsection{Determination of $V_{b}$ from the color of the LR detector}

$\mathrm{Yu}$ and $\mathrm{Ng}$ [44] proposed a method based on a color commercial document scanner to determine the active layer thickness of the LR 115 SSNTD. They found a hyperbolic relationship between the optical density at the R band and the thickness of the active layer for LR 115 detector.

\subsubsection{Comments}

Although the methods described in this section are easy to use, these are indirect methods. Direct methods for $V_{\mathrm{b}}$ determination are based on digital micrometers, atomic force microscopes or surface profilometers. Direct measurements of $V_{\mathrm{b}}$ will be described in the next section.

Since $V_{\mathrm{b}}$ is one of the most important parameters that control the formation and development of tracks, there has been a large volume of literature discussing $V_{\mathrm{b}}$. Here, an incomplete survey of works on $V_{\mathrm{b}}$ is given. It has been shown that $V_{\mathrm{b}}$ depends on many factors like the purity of the basic substances, the molecular structures of polymers, conditions of polymerization, environmental conditions during the irradiation and finally on etching conditions [45]. In this way, there are different topics related to $V_{\mathrm{b}}$, which include the following:

- dependence of $V_{\mathrm{b}}$ on the chemical composition and preparation of the detector;

- dependence of $V_{\mathrm{b}}$ on the etching conditions (temperature and concentration of etching solution as well as the presence of stirring);

- dependence of $V_{\mathrm{b}}$ on the irradiation of the detector before etching with different kinds of ionizing or non-ionizing radiations. 
Table 1

Experimental $V_{\mathrm{b}}$ data as a function of the temperature for the CR-39 detector using $6.25 \mathrm{~N} \mathrm{NaOH}$ as the etchant [47]

\begin{tabular}{ll}
\hline Water bath temperature $\left({ }^{\circ} \mathrm{C}\right)$ & $V_{\mathrm{b}}(\mu \mathrm{m} / \mathrm{h})$ \\
\hline 26 & 0.036 \\
40 & 0.125 \\
54 & 0.46 \\
70 & 1.59 \\
\hline
\end{tabular}

\subsection{7. $V_{b}$ dependence on etching conditions}

This topic is very often investigated. The dependence of $V_{\mathrm{b}}$ was studied for different detectors and for different etching conditions. It has been established that the etching rate of plastic track detectors increases exponentially with the etching temperature $T$ [4] as shown in Eq. (3.4):

$$
V=V_{0} \mathrm{e}^{-\varepsilon_{0} / k T}
$$

where $T$ is the temperature in $\mathrm{K}, \varepsilon_{0}$ is the activation energy in $\mathrm{eV}, k$ is the Boltzmann's constant and $V_{0}$ is a proportionality constant. According to Adams [46], Eq. (3.4) is applicable to both $V_{\mathrm{b}}$ and $V_{\mathrm{t}}$ with different activation energies. The activation energy for the CR-39 detector is in the range of $0.7-1 \mathrm{eV}$.

Fowler et al. [47] used measurements of the minor axis of openings of particle tracks formed under oblique incidence to measure $V_{\mathrm{b}}$. Their experimental $V_{\mathrm{b}}$ data as a function of the temperature for the CR-39 detector using $6.25 \mathrm{~N} \mathrm{NaOH}$ as the etchant are shown in Table 1. The measurements were made for the Pershore Moulding CR-39 detectors cured for 16 and $36 \mathrm{~h}$, and no important differences were found between them. The activation energy obtained in this experiment was $0.78 \pm$ $0.03 \mathrm{eV}$.

Gruhn et al. [48] investigated the etching mechanism and the effects of molarity of $\mathrm{KOH}$ and $\mathrm{NaOH}$ on Lexan and CR-39 detectors at $70{ }^{\circ} \mathrm{C}$. The mechanism was the chemical attack of the $\mathrm{OH}^{-}$ group resulting in hydrolysis of the carbonate ester bonds in the polymer. In those experiments, the CR-39 detectors were obtained from American Acrylic and Plastics Inc. and $V_{\mathrm{b}}$ was determined by the diameter of tracks from fission fragments. Exponential growth of $V_{\mathrm{b}}$ with molarity was found in all investigated cases. The data are given in Table 2.

Al-Najjar et al. [49] also measured the bulk etch rate in the CR-39 detector using fission fragments of ${ }^{252} \mathrm{Cf}$ for normal-incidence irradiation. Etching was performed in $\mathrm{NaOH}$ and $\mathrm{KOH}$ solutions. The bulk etch rate was found to increase exponentially with the etchant concentration and decrease linearly with $1 / T$. The activation energy was determined as $0.74 \mathrm{eV}$.

Somogyi and Hunyadi [50] also investigated the etching properties of the CR-39 detector. The bulk etch rate was measured from the diameter $d_{\mathrm{f}}$ of the tracks related to ${ }^{252} \mathrm{Cf}$ fission fragments

Table 2

$V_{\mathrm{b}}$ for CR-39 as a function of molarity of $\mathrm{NaOH}$ and $\mathrm{KOH}$ [48]

\begin{tabular}{llcc}
\hline Molarity of $\mathrm{NaOH}$ & $V_{\mathrm{b}}(\mu \mathrm{m} / \mathrm{h})$ & Molarity of $\mathrm{KOH}$ & $V_{\mathrm{b}}(\mu \mathrm{m} / \mathrm{h})$ \\
\hline 3.92 & 0.70 & 1.89 & 0.40 \\
4.90 & 0.98 & 3.78 & 1.14 \\
5.88 & 1.26 & 4.72 & 1.66 \\
7.84 & 2.04 & 5.67 & 2.21 \\
9.80 & 3.32 & 7.56 & 4.03 \\
11.8 & 5.5 & 9.45 & 8.16 \\
- & - & 11.8 & 18 \\
\hline
\end{tabular}




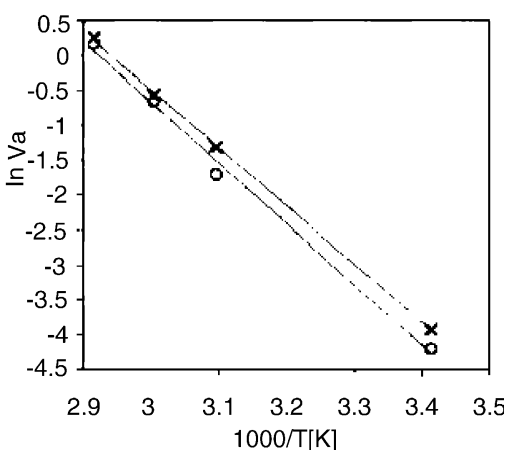

Fig. 13. Variation of the bulk etch rate in CR-39 detectors produced in Italy (circles) and Hungary (crosses) [39].

normally incident on the detector surface from the equation $V_{\mathrm{b}}=d_{\mathrm{f}} / 2 t$. It was found that the bulk etch rate can be described by the relation:

$$
V_{\mathrm{b}}=f_{\mathrm{B}} C^{n_{\mathrm{B}}} \mathrm{e}^{-\varepsilon / k T}
$$

where $T$ is the temperature in $\mathrm{K}, C$ is the concentration of the etchant in the unit of normality and $k$ is the Boltzman's constant. The constants $f_{\mathrm{B}}, n_{\mathrm{B}}$ and the activation energy $\varepsilon$ were obtained from fitting procedures as $f_{\mathrm{B}}=(8.2 \pm 0.4) \times 10^{11} \mu \mathrm{m} / \mathrm{h}, n_{\mathrm{B}}=3 / 2$ and $\varepsilon=0.88 \pm 0.04 \mathrm{eV}$. The difference between Eqs. (3.4) and (3.5) is the appearance of the term $C^{n}$ in the latter which accounts for the concentration of the etchant.

The gravimetric method for measuring the bulk etch rate was used by Kocsis et al. [39]. Variation of $V_{\mathrm{b}}$ for a $5 \mathrm{M} \mathrm{NaOH}$ etching solution is given as a function of 1/T for two types of CR-39 detectors (Italian and Hungarian) in Fig. 13. The activation energy was found similar for these two detectors: $0.72 \mathrm{eV}$ for the Hungarian made and $0.75 \mathrm{eV}$ for the Italian made detectors.

Ansari et al. [51] measured $V_{\mathrm{b}}$ and $V_{\mathrm{t}}$ for CR-39 detectors manufactured by Pershore Moulding Ltd. in UK and American Acrylic and Plastics Inc., and in SR-86 detectors. The CR-39 detectors were etched in a $6 \mathrm{M} \mathrm{NaOH}$ solution at $70{ }^{\circ} \mathrm{C}$, and $V_{\mathrm{b}}=1.1 \mu \mathrm{m} / \mathrm{h}$ was found for the detectors from American Acrylic and Plastics Inc. while $V_{\mathrm{b}}=1.2 \mu \mathrm{m} / \mathrm{h}$ for the detectors from Pershore Moulding Ltd. in UK. The bulk etch rate in SR-86, etched in $7 \mathrm{M} \mathrm{NaOH}$ at $70{ }^{\circ} \mathrm{C}$ was about three times larger, amounting to $3.4 \mu \mathrm{m} / \mathrm{h}$. Significant ageing effects (discussed further) were discovered in the SR-86 detector after 1 year of storage, where the detectors were completely fogged and totally destroyed.

A formula which was slightly different from Eq. (3.5) was found by Fromm et al. [52] who studied the bulk etch of CR-39 for different concentrations and temperatures of the $\mathrm{NaOH}$ solution. They proposed the function for $V_{\mathrm{b}}$ of the CR-39 detector as:

$$
V_{\mathrm{b}}=1.276 \mathrm{e}^{[0.828 C+0.049 T-0.002 C T-17.624]}
$$

where $T$ was the temperature expressed in $K$ and $C$ was the concentration of the etchant in mol/l. This fitting formula is different from Eq. (3.5) in that $V_{\mathrm{b}}$ now has an exponential growth with the concentration $C$, whereas Eq. (3.5) predicts a power increment of $V_{\mathrm{b}}$ with $C$. Furthermore, there is no explicit dependence on the activation energy in this equation.

A survey of previously reported values of the bulk etch activation energy in CR-39 detectors was given by Awad and El-Samman [53]. Their table is reproduced here as in Table 3 (with the activation energy denoted as $E_{\mathrm{b}}$ ). 
Table 3

Survey of bulk etch activation energies $E_{\mathrm{b}}$ in CR-39 detectors [53]

\begin{tabular}{|c|c|c|c|c|}
\hline CR-39 & Ion beam & $\gamma$ & $E_{\mathrm{b}}(\mathrm{keV})$ & $E_{\mathrm{b}}(\mathrm{keV})$ \\
\hline Pitsburgh & & & $0.85 \pm 0.05$ & \\
\hline a & $\mathrm{ff}$ & & 0.92 & 0.68 \\
\hline Homalite & Alpha particles & & $0.885 \pm 0.04$ & $0.88 \pm 0.04$ \\
\hline Homalite & & Yes & $0.83 \pm 0.04$ & \\
\hline Pershore & & & $0.78 \pm 0.03$ & \\
\hline Pershore & & & $0.786 \pm 0.02$ & \\
\hline Pershore & & & $0.77 \pm 0.02$ & \\
\hline Pershore & & & $0.792 \pm 0.024$ & \\
\hline Pershore & & & $0.799 \pm 0.013$ & \\
\hline
\end{tabular}

References for the data were given in [53].

a The type of CR-39 used is unknown.

The same authors also measured $V_{\mathrm{b}}$ and the bulk etch activation energy in the CR-39 detector. The bulk etch rate was measured by mass difference. The dependence of $V_{\mathrm{b}}$ on temperature was found to satisfy the Arhenius type equation. The dependence of $V_{\mathrm{b}}$ on temperature $T$ was shown by plotting $\ln V_{\mathrm{b}}$ as a function of $1 / T$. From the slope of the lines, one could derive the activation energy $E_{\mathrm{b}}$. It was found that $E_{\mathrm{b}}=0.76 \pm 0.03 \mathrm{eV}$. The activation energy was similar for CR-39 detectors provided by different manufacturers.

Szydlowski et al. [54] determined $V_{\mathrm{b}}$ in three different types of detectors, namely, CR-39, PM-344 and PM-500, obtained from Pershoure Moulding Ltd. All detectors had the same chemical composition, and differed only in additives, dopants, plasticizer and curing cycles. The etchant was $6.25 \mathrm{~N} \mathrm{NaOH}$ at $70{ }^{\circ} \mathrm{C}$. They found $V_{\mathrm{b}}=0.92 \pm 0.08 \mu \mathrm{m} / \mathrm{h}$ for all three types of detectors.

The bulk etch rate was also measured for other types of detectors (i.e., not only CR-39 detectors). For example, Gruhn et al. [48] measured $V_{\mathrm{b}}$ for the Kodak Pathe cellulose nitrate plastic detector as a function of temperature and normality of the etching solution. The normality of $\mathrm{NaOH}$ was varied between 2.5 and $7.69 \mathrm{~N}$ and the temperature was varied between 25 and $55^{\circ} \mathrm{C}$. The characteristics of $V_{\mathrm{b}}$ growth with the normality depended on the temperature. At $25^{\circ} \mathrm{C}$, the growth was represented as exponential, but at higher temperatures the growth was not exponential and there was a saturation effect for larger normality. The track etch rate also showed similar behavior and saturation effect with the normality.

The bulk etch rates for Makrofol detectors ( $\mathrm{KG}, \mathrm{KL}$ and $\mathrm{N}$, polycarbonate (bisphenol-A polycarbonate: $\mathrm{C}_{16} \mathrm{H}_{14} \mathrm{O}_{3}$ )) were measured by Kumar and Prasad [55] where etching was performed in $6.25 \mathrm{~N} \mathrm{KOH}$ at temperatures $55,60,65$ and $70{ }^{\circ} \mathrm{C}$. The results for the bulk etch rate are given in Table 4.

The variation of the bulk etch rate with temperature follows the Arhenius type of law. The bulk activation energy was determined as $0.99 \pm 0.04 \mathrm{eV}$ in Makrofol KG, $1.18 \pm 0.08 \mathrm{eV}$ in Makrofol KL and $0.89 \pm 0.04 \mathrm{eV}$ in Makrofol $\mathrm{N}$ detectors.

\subsubsection{Influence of detector ageing}

The age of the detector can affect the bulk etch rate. It has also been shown that the effects of age are different in CR-39 and LR 115 detectors.

Cecchini et al. [56] measured $V_{\mathrm{b}}$ in CR-39 detectors manufactured by Intercast Europe Co. (Parma, Italy). Large area detectors were intended for search of magnetic monopoles in the Gran Saso Laboratory in Italy. The duration of the experiment was planned for several years and the variations of 
Table 4

Bulk etch rates in different types of Makrofol polycarbonate detectors etched in $6.25 \mathrm{~N} \mathrm{KOH}$ at different temperatures [55]

\begin{tabular}{lllr}
\hline Temperature $\left({ }^{\circ} \mathrm{C}\right)$ & \multicolumn{2}{l}{ Bulk etch rate $(\mu \mathrm{m} / \mathrm{h})$} & Makrofol N \\
\cline { 2 - 4 } & Makrofol KG & Makrofol KL & $0.42 \pm 0.05$ \\
\hline 55 & $0.31 \pm 0.04$ & $0.19 \pm 0.07$ & $0.66 \pm 0.05$ \\
60 & $0.54 \pm 0.06$ & $0.36 \pm 0.06$ & $1.24 \pm 0.05$ \\
65 & $1.08 \pm 0.03$ & $0.84 \pm 0.08$ & $1.66 \pm 0.08$ \\
70 & $1.44 \pm 0.06$ & $1.20 \pm 0.07$ & \\
\hline
\end{tabular}

detector characteristics with the age were important in this experiment. Detectors of different ages were etched in $6 \mathrm{~N} \mathrm{NaOH}$ solution at $70{ }^{\circ} \mathrm{C}$, and $V_{\mathrm{b}}$ was determined to vary from 1.092 up to $1.231 \mu \mathrm{m} /$ $\mathrm{h}$. The variation of $V_{\mathrm{b}}$ was explained by the different ages of the various samples. From this experiment, it may be concluded that the effects from age are relatively small in CR-39 detectors.

Siems et al. [57] measured the bulk etch rate $V_{\mathrm{b}}$ of LR 115 detectors as a function of the detector age. Their results are summarized in Table 5.

Etching was performed in $10 \%$ aqueous solution of $\mathrm{NaOH}$ at $59.2{ }^{\circ} \mathrm{C}$. Depth dependence of $V_{\mathrm{b}}$ was observed in some samples but not in others. For this reason, the results were specified for the top $4 \mu \mathrm{m}$ of the detector and for the rest of the detector (inner layer). Detectors older than 5 years seemed to show odd behaviors of $V_{\mathrm{b}}$. The trend is that $V_{\mathrm{b}}$ decreases with increasing age. This finding may be important in applications of this detector. For example, the calibration factor for radon measurements should be established by taking into account the age of the detector.

\subsubsection{Influence of irradiation of detector on $V_{b}$}

The bulk etch rate depends on the irradiation of the detector before etching. Investigations include those on the effects of irradiation to gamma rays, electrons, protons, ultraviolet and infrared radiation. The common finding is the increase in $V_{\mathrm{b}}$ with the dose of radiation.

Abu-Jarad et al. [58] studied the effect of high gamma dose on the response of PADC detector (CR-39). They measured the bulk etch rate $V_{\mathrm{b}}$, alpha track etch rate $V_{\mathrm{t}}$ and the sensitivity $V=V_{\mathrm{t}} / V_{\mathrm{b}}$ based on measurements of the track diameters for normally incident fission fragments and alpha

Table 5

Bulk etch rate of the LR 115 detector as a function of the age [57]

\begin{tabular}{llll}
\hline Detector & Thickness $(\mu \mathrm{m})$ & Bulk etch rate $(\mu \mathrm{m} / \mathrm{h})$ & Depth $(\mu \mathrm{m})$ \\
\hline LR 115 (February 1981) & $12.29 \pm 0.14$ & $3.22 \pm 0.08$ & $0-4$ (Top layer) \\
& & $3.73 \pm 0.15$ & 4-12 (Inner layer) \\
LR 115 (November 1986) & $12.25 \pm 0.19$ & $2.95 \pm 0.07$ & $0-12$ \\
LR 115 (February 1988) & $12.67 \pm 0.17$ & $3.12 \pm 0.08$ & $0-12$ \\
LR 115 (July 1990) & $11.67 \pm 0.13$ & $2.90 \pm 0.09$ & $0-12$ \\
LR 115 (September 1994) & $11.44 \pm 0.10$ & $4.46 \pm 0.07$ & $0-4$ (Top layer) \\
& & $5.57 \pm 0.18$ & 4-12 (Inner layer) \\
LR 115 (February 1999) & $11.61 \pm 0.10$ & $3.03 \pm 0.12$ & $0-4$ (Top layer) \\
& & $5.32 \pm 0.14$ & 4-12 (Inner layer) \\
LR 115 (September 1994) & $11.40 \pm 0.07$ & $4.21 \pm 0.07$ & $0-4$ (Top layer) \\
& & $5.63 \pm 0.27$ & 4-12 (Inner layer) \\
\hline
\end{tabular}




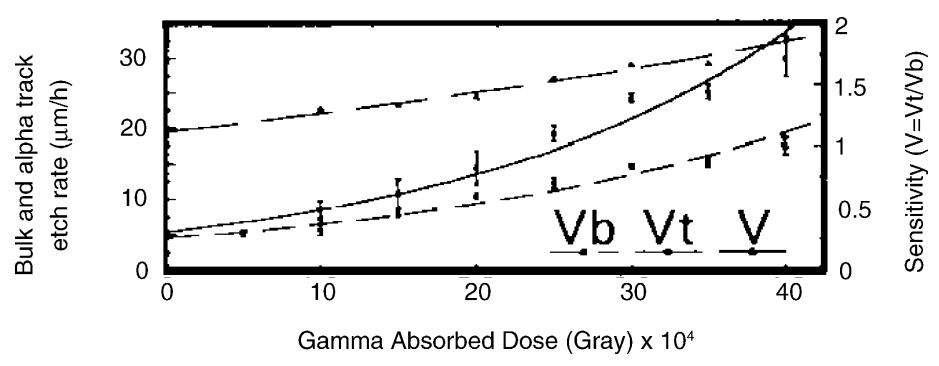

Fig. 14. Dependency of the bulk etch rate $V_{\mathrm{b}}$, alpha track etch rate $V_{\mathrm{t}}$ and sensitivity $V$ on the gamma dose for the PADC detector [58].

particles according to the following equation:

$$
V_{\mathrm{b}}=\frac{D_{\mathrm{ff}}}{2 t}, \quad x=\frac{D_{\alpha}}{D_{\mathrm{ff}}}, \quad V=\frac{1+x^{2}}{1-x^{2}}, \quad V_{\mathrm{t}}=V V_{\mathrm{b}}
$$

where $D_{\mathrm{ff}}$ was the diameter of tracks resulting from the fission fragments and $D_{\alpha}$ was the diameter of alpha tracks. Etching was performed for $30 \% \mathrm{KOH}$ at $60{ }^{\circ} \mathrm{C}$. The PM-355 detectors obtained from Pershore Moulding Ltd. (UK) were firstly irradiated to alpha particles and fission fragments, and subsequently to gamma rays from a ${ }^{60}$ Co source. All parameters, $V_{\mathrm{b}}$ and $V_{\mathrm{t}}$, increase with the doses as shown in Fig. 14.

The effect of gamma dose on the PADC detector was also studied by Sinha et al. [59]. Detectors were irradiated by alpha particles and fission fragments from a ${ }^{252} \mathrm{Cf}$ source, and gamma rays from a ${ }^{60} \mathrm{Co}$ source. Etching was performed using $6 \mathrm{~N} \mathrm{NaOH}$ at 55, 60, 65 and $70{ }^{\circ} \mathrm{C}$. According to these authors, $V_{\mathrm{b}}$ and $V_{\mathrm{t}}$ remain unchanged with a gamma dose up to $10^{4} \mathrm{~Gy}$, then both increase slowly until $10^{5} \mathrm{~Gy}$, and then increase sharply. Similar results were found in post- and pre-gamma exposure with the difference that both $V_{\mathrm{b}}$ and $V_{\mathrm{t}}$ are higher in the case of post-gamma exposure. The curves obtained by these authors are shown in Fig. 15.

Yamauchi et al. [60] also investigated the effects of gamma rays and pulsed electrons on the bulk etch rate of CR-39 detectors. The doses were up to $100 \mathrm{kGy}$ and the dose rate varied from 0.0044 up to $35 \mathrm{~Gy} / \mathrm{s}$. The bulk etch rate increased exponentially with the dose for all investigated dose rates. It was also discovered that the bulk etch rate decreases with the dose rate for the same total dose. The bulk etch rate was measured from the diameter of tracks from ${ }^{252} \mathrm{Cf}$ fission products. The bulk etch rate of

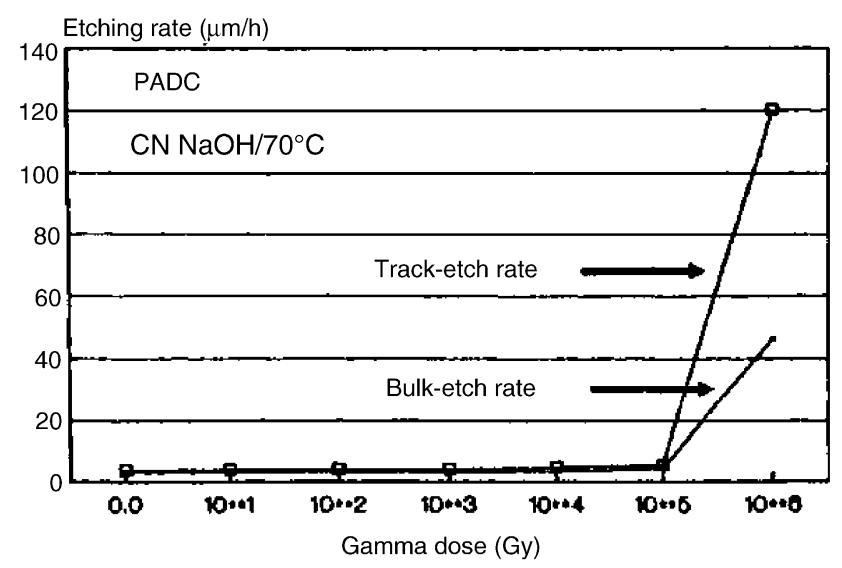

Fig. 15. Effect of post-gamma dose on $V_{\mathrm{b}}$ and $V_{\mathrm{t}}$ of PADC [59]. 
irradiated samples $V_{\text {irra }}$ was found to satisfy the equation:

$$
V_{\text {irra }}=V_{\mathrm{b}} \exp (g D)
$$

where $V_{\mathrm{b}}$ was the bulk etch rate of non-irradiated samples and $D$ was the absorbed dose. For etching in stirred $6 \mathrm{~N} \mathrm{KOH}$ solution at $70{ }^{\circ} \mathrm{C}, V_{\mathrm{b}}$ was determined as $2.36 \mu \mathrm{m} / \mathrm{h}$. The factor $g$ was dose dependent and was determined from least square fitting of the experimental data.

Yamauchi et al. [61] irradiated Baryotrak CR-39 detectors with fission fragments from ${ }^{252} \mathrm{Cf}$. The fission products were arranged to impact on a lateral polished surface of the detector for investigation of the track profile. Subsequently, the detectors were irradiated to gamma rays from a ${ }^{60} \mathrm{Co}$ source with different doses and different dose rates. Bottle-like tracks of fission products were discovered, which indicated strong depth dependence of the bulk etch rate. The thickness of the layer damaged by gamma rays increases with decreasing dose rate. From these experiments, the authors proposed a gamma dosimeter based on the CR-39 detector. Such a dosimeter may be applicable in high dose environments. The dose rate may be determined from the thickness of the damaged surface layer of the detector.

Fazal-ur-Rehman et al. [62] compared three methods for determining the bulk etch rate in PM355 detectors irradiated with high gamma doses. They compared the thickness change method, mass change method and fission track diameter method. The detectors were irradiated by gamma rays from a ${ }^{60} \mathrm{Co}$ source with the dose in the range $10^{5}-1.2 \times 10^{6} \mathrm{~Gy}$. Etching was performed in $30 \% \mathrm{KOH}$ at $70{ }^{\circ} \mathrm{C}$. The bulk etch rate as a function of the total dose for constant etching time is shown in Fig. 16. On the other hand, the bulk etch rate as a function of etching time for a fixed dose is given in Fig. 17.

One can see from Figs. 16 and 17 that the bulk etch rates determined from the thickness change and mass change methods agreed well. However, the track diameter method was not successful under such high gamma doses. In conclusion, $V_{\mathrm{b}}$ increased with the absorbed gamma dose (which agrees with Figs. 14 and 15) and decreased with the depth.

The effects of high gamma doses and electron irradiation on the bulk etch rate of the PM-355 detector was studied by Szydlowski et al. [63]. Etching was performed in $6.25 \mathrm{~N}$ aqueous solution of $\mathrm{NaOH}$ at $70{ }^{\circ} \mathrm{C}$, and the bulk etch rate was measured from the mass changes. The results are given in Fig. 18.

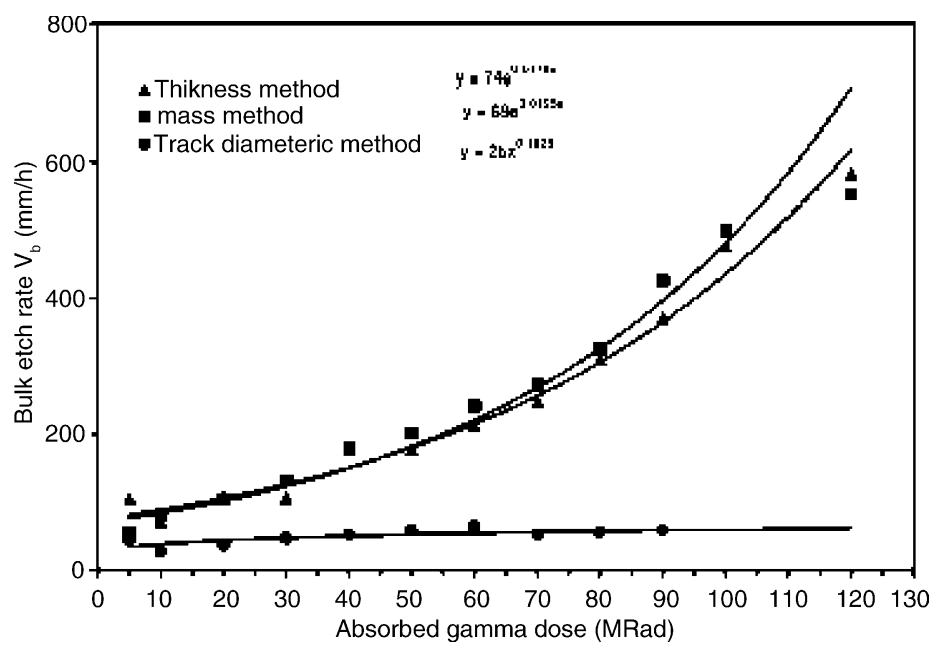

Fig. 16. The bulk etch rate of PM-355 detector as a function of the absorbed dose determined from three methods for a fixed etching time of $8.5 \mathrm{~min}[62]$. 


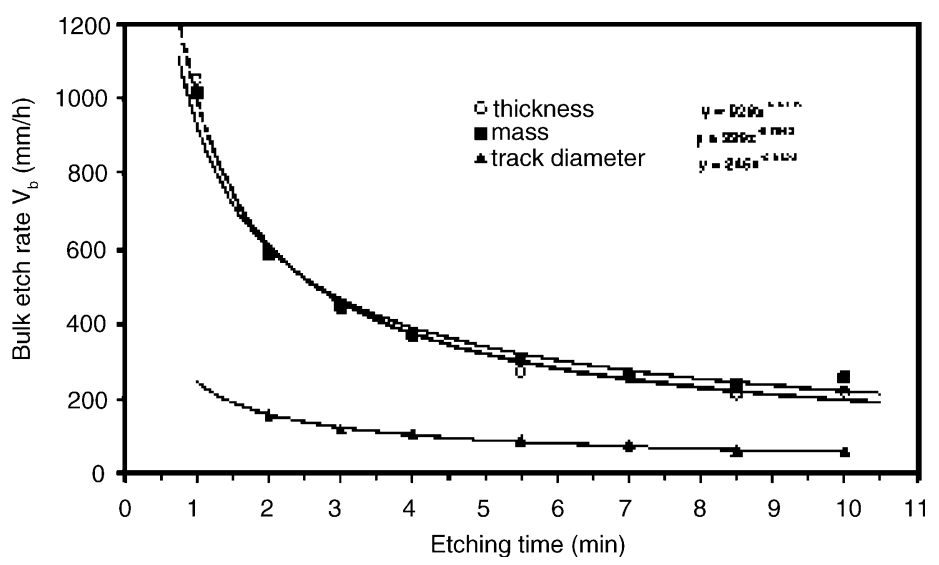

Fig. 17. The bulk etch rate of PM-355 detector as a function of the etching time for a fixed gamma dose of $60 \mathrm{Mrad}$ [62].

Mishra et al. [64] also observed an increase in the bulk etch rate in polyethylene terephthalate and polyimide after irradiation to $2 \mathrm{MeV}$ electrons. In addition to the increase of the bulk etch rate, surface modification was also noticed. The surface became rough under the influence of the gamma and electron irradiation, which made observation of tracks more difficult.

Ultraviolet radiation also affects the detector response and $V_{\mathrm{b}}$. For example, Abu-Jarad et al. [65] studied the effects of solar ultraviolet radiation on the CR-39 detector characteristics, and found that $V_{\mathrm{b}}$ increases with the irradiation to solar ultraviolet radiation. As a result, the diameter of alpha tracks also increases with the irradiation. From this finding, a possibility for using CR-39 detectors as ultraviolet detectors was revealed. The effects of ultraviolet and laser radiation on different batches of CR-39 were also investigated by Abu-Jarad et al. [66]. Increases in $V_{\mathrm{b}}$ and $V_{\mathrm{t}}$ were observed with irradiation of the CR-39 detectors to ultraviolet radiation. A small increase of $V_{\mathrm{b}}$ and $V_{\mathrm{t}}$ after short irradiation (up to $40 \mathrm{~s}$ ) to laser beam was also observed. However, prolonged irradiation to laser produced color changes, damages and burning of the detector surface.

It was also found that irradiation with proton beams changed the CR-39 detector response. The bulk etch rate of PADC CR-39 detector etched in $6 \mathrm{~N} \mathrm{NaOH}$ at $55{ }^{\circ} \mathrm{C}$ increased from $1 \mu \mathrm{m} / \mathrm{h}$ (for

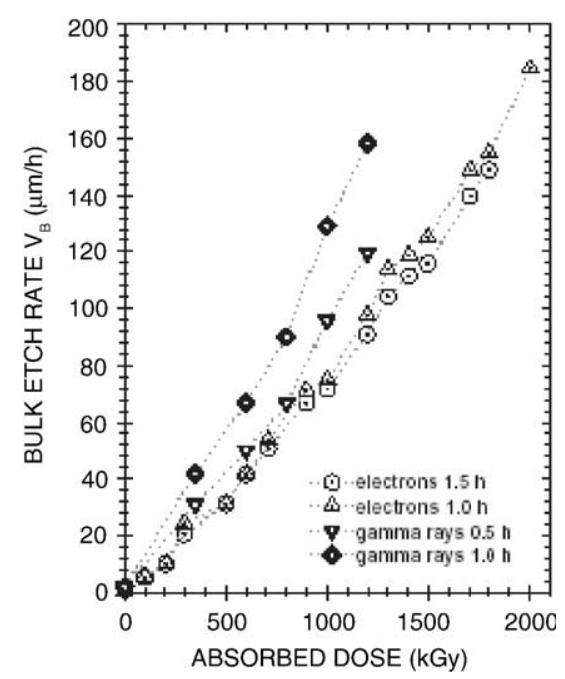

Fig. 18. The bulk etch rate of PM-355 detectors exposed to ${ }^{60} \mathrm{Co}$ gamma rays, and $10 \mathrm{MeV}$ electrons [63]. 


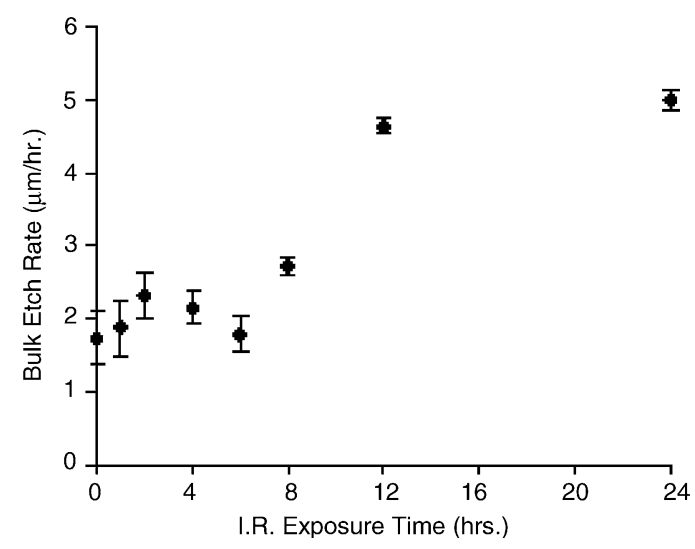

Fig. 19. Bulk etch rate as a function of exposure time of infrared radiation [69].

non-irradiated samples) to $1.3 \mu \mathrm{m} / \mathrm{h}$ (for samples irradiated with a dose $D=10^{4}$ Gy from $62 \mathrm{MeV}$ proton beam) [67]. Influence of $62 \mathrm{MeV}$ proton irradiation on the Makrofol $\mathrm{N}$ detector and polyimide was studied by Tripathy et al. [68] and the bulk etch rate was found to increase in both examined detectors with the proton dose, while the activation energy decreased.

Prasher and Singh [69] investigated the effects of infrared light on the CR-39 detector. CR-39 detectors (from Pershore Moulding Ltd.) were etched in $6.25 \mathrm{~N} \mathrm{NaOH}$ solution at $70{ }^{\circ} \mathrm{C}$ and $V_{\mathrm{b}}$ was measured from direct measurements with a digimatic micrometer. The samples were irradiated by an infrared lamp of $150 \mathrm{~W}$ at a distance of $6 \mathrm{~cm}$ for different periods from 1 to $24 \mathrm{~h}$. The results obtained are given in Fig. 19.

The increase in the bulk etch rate was explained on the basis of scission of hydrocarbon chains that enhanced the dissolution rate. Furthermore, the track etch rate increased in a similar way as the bulk etch rate.

\subsubsection{Direct measurements of $V_{b}$}

As mentioned earlier, direct measurements of $V_{\mathrm{b}}$ can be made with a digital micrometer, atomic force microscope or surface profilometer. Measurements of $V_{\mathrm{b}}$ with the micrometer are based on the determination of the thickness of the removed layer during etching. The problem that arises here is the impossibility to apply the same pressure on the detector in two separate measurements.

Nikezic and Janicijevic [70] used a surface profilometer (with the brand name Form Talysurf) to measure $V_{\mathrm{b}}$ in LR 115 detectors. One part of the active layer was removed by a razor. The stylus of the surface profilometer scanned across this cliff of the active layer over the polyester base. The difference between the two levels gave the height of the cliff which was the residual thickness of the detector, from which $V_{\mathrm{b}}$ could be determined. It was found that $V_{\mathrm{b}}=3.27 \mu \mathrm{m} / \mathrm{h}$ for etching under $2.5 \mathrm{~N} \mathrm{NaOH}$ at $60{ }^{\circ} \mathrm{C}$. A typical output from Form Talysurf is shown in Fig. 20. The removed layer as a function of etching time is given in Fig. 21. The bulk etch rates derived from the slope of lines in Fig. 21 are given in Table 6.

The masking method for bulk etch rate measurements was introduced by Yasuda et al. [71]. Before etching, part of a piece of CR-39 detector was masked by epoxy which was a material resistant to the etchant. After etching, the masked part of the detector remained unetched. The epoxy mask was removed and the bulk etch could then be determined by the difference in the heights of the exposed part and the masked part of the detector. Then, surface profilometry was employed to determine this height difference. The masking method together with surface profilometery measurements were made 


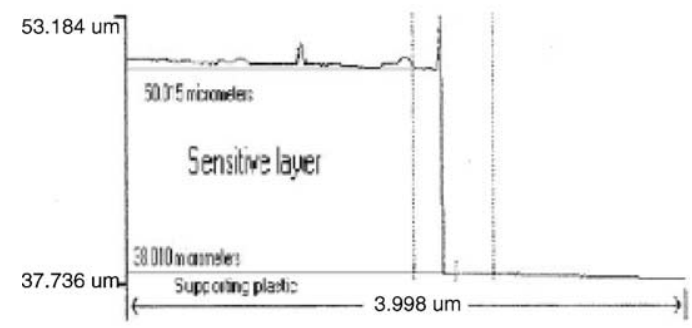

Fig. 20. An output from the Form Talysurf (Taylor Hobson) surface profilometer [70]. The figure represents the measurement of the initial thickness (before etching) of a piece of LR 115 detector. The thickness of this sample is calculated as $50.015-38.010=12.005 \mu \mathrm{m}$. The stylus has scanned a horizontal distance of $3.988 \mathrm{~mm}$.

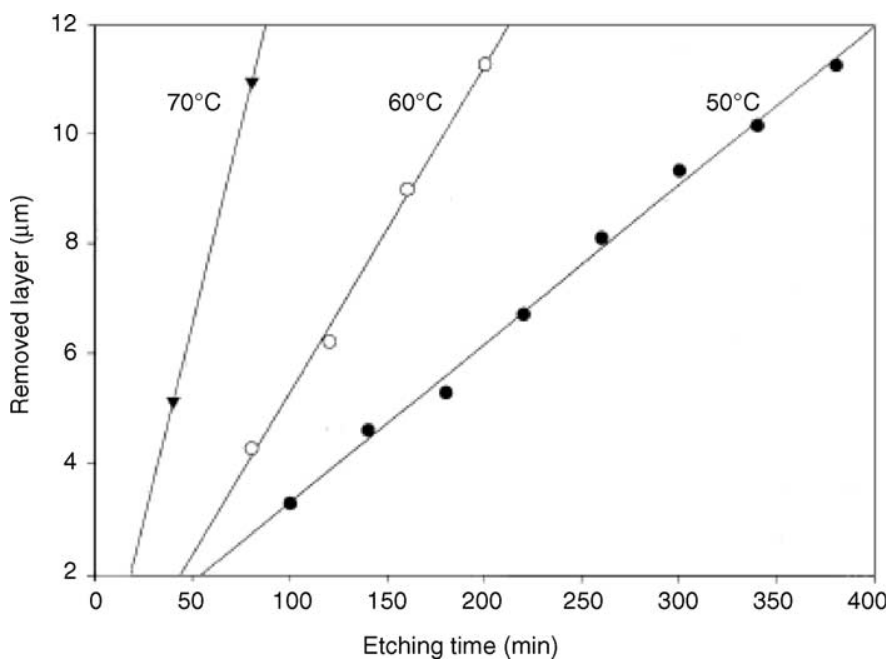

Fig. 21. The removed layer of the LR 115 detector as a function of the etching time, with the etching temperature as parameter [70].

by Ho et al. [72] to study the effect of stirring on the bulk etch rate of the CR-39 detector. An example of a three-dimensional profile for a CR-39 detector after etching for $2 \mathrm{~h}$ without stirring is given in Fig. 22.

In order to investigate the effect of stirring of the etchant, magnetic stirring was applied. The detectors were etched in $6.25 \mathrm{~N} \mathrm{NaOH}$ maintained at $70{ }^{\circ} \mathrm{C}$ by a water bath. One set of detectors were etched under no stirring while the other set of detectors were etched using a magnetic stirrer. At each of the selected time intervals, i.e., $0.5,1,2,3,4$ and $5 \mathrm{~h}$, for both etching under magnetic stirring and under no stirring, a piece of CR-39 detector was taken out from the corresponding beaker. The detectors were immediately rinsed by deionized water and the epoxy masks were removed at the same

Table 6

The bulk etch rates of the LR 115 detector at various etching temperature of $10 \% \mathrm{NaOH}$

\begin{tabular}{lc}
\hline Temperature of the etchant $10 \% \mathrm{NaOH}\left({ }^{\circ} \mathrm{C}\right)$ & Etching rate $(\mu \mathrm{m} / \mathrm{h})$ \\
\hline 50 & $1.75 \pm 0.03$ \\
60 & $3.27 \pm 0.08$ \\
70 & $8 \pm 0.3$ \\
\hline
\end{tabular}




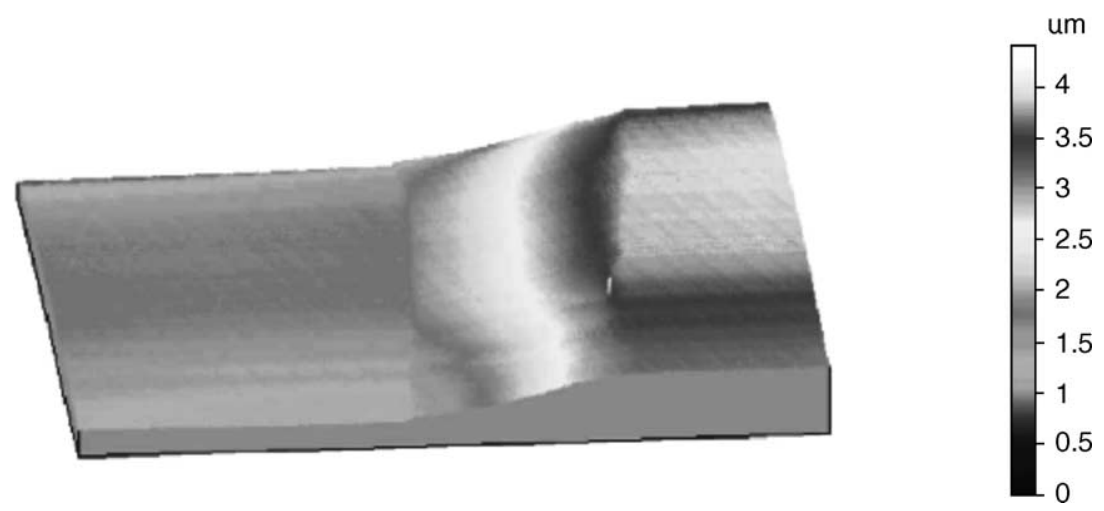

Fig. 22. The three-dimensional profile for a CR-39 detector after etching for $2 \mathrm{~h}$ without stirring obtained by Form Talysurf PGI [72].

time. The bulk etches were then determined using surface profilometry. The results are presented in Fig. 23.

The bulk etch rates can be determined as the slopes of lines in Fig. 23. The results were found as follows:

- $B=1.104 \pm 0.024$ for etching under magnetic stirring;

- $B=1.195 \pm 0.028$ for etching under no stirring.

In other words, the bulk etch rates were $1.10 \pm 0.02$ and $1.20 \pm 0.03 \mu \mathrm{m} / \mathrm{h}$ for etching under magnetic stirring and under no stirring, respectively. These results suggested that stirring was of little influence on bulk etch rate of the CR-39 detector.

In contrary to this finding, stirring was found to have a great influence on the bulk etch rate of the LR 115 detector. Yip et al. [73] measured the bulk etch rate for the LR 115 detector (with and without stirring), also by means of surface profilometry. Etching was performed in $10 \%$ aqueous solution of $\mathrm{NaOH}$ at $60{ }^{\circ} \mathrm{C}$. The bulk etch rate under magnetic stirring was found to be $6.65 \pm 0.34 \mu \mathrm{m} / \mathrm{h}$ while that under no stirring was $3.61 \pm 0.14 \mu \mathrm{m} / \mathrm{h}$.

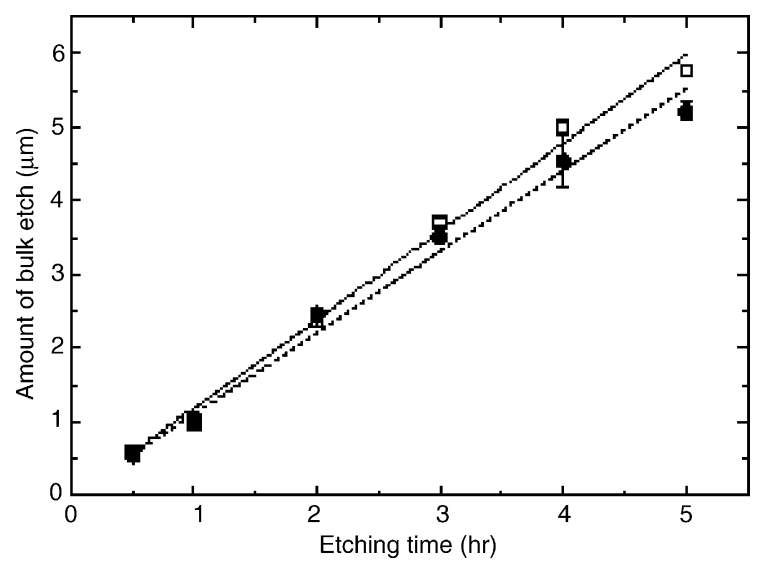

Fig. 23. Relationship between the amount of bulk etch ( $\mu \mathrm{m})$ (measured by Form Talysurf) and the etching time (h) for the CR-39 detector. Open squares and solid line: data obtained with etching under no stirring and the corresponding best fitting line; solid circles and dashed line: data obtained with etching under magnetic stirring and the corresponding best fitting line [72]. 
By using the Form Talysurf surface profilometer, Yip et al. [74] studied the inhomogenity in the thickness of the LR 115 detector and found that the coefficient of variation has risen from $1.7 \%$ before etching to $8 \%$ after $2 \mathrm{~h}$ of etching. They explained the increased inhomogenity by the formation of track-like damages, which were observed using surface profilometry, SEM and optical microscopy [74] as well as atomic force microscopy [75].

\subsubsection{AFM studies of bulk etch rate and surface roughness}

Application of the atomic force microscope (AFM) is a relatively new technique for track studies. The survey of different kinds of scanning microscopes and their applications in track studies were given by Vukovic and Atanasijevic [76]. AFM enabled very accurate measurements of the bulk etch rate by the "masking" method $[71,77]$. Part of the detector was masked in order to prevent etching of that surface. After a short etching, the mask was removed and the profile of the surface was recorded by AFM in the tapping mode. This technique enabled measurements of very thin removed layers. A typical image is given in Fig. 24.

From Fig. 24, one can conclude that $V_{\mathrm{b}}$ is about $2.4 \mu \mathrm{m} / \mathrm{h}$. In the same paper, the sizes of the minutely developed tracks of different ions were measured. However, care should be taken in the measurements of the track depth and the track profile with the AFM, since the geometrical incompatibility between the tracks and the AFM probe could lead to inaccuracies [78].

Vazquez-Lopez et al. [79] used AFM to study the surface roughness and the bulk etch rate for different kinds of CR-39 detectors. The surfaces of the detectors before etching were different. The bulk etch rate for etching in $6.25 \mathrm{M} \mathrm{KOH}$ solution at $60 \pm 1{ }^{\circ} \mathrm{C}$ was found as $1.13 \pm 0.01,1.14 \pm 0.01,0.89$ \pm 0.01 and $1.03 \pm 0.01 \mu \mathrm{m} / \mathrm{h}$ for the detectors from American Acrylic and Plastics Inc., Fujii, Lantrack and Pershore Moulding Ltd., respectively. The alpha-particle tracks were observed after etching for $3 \mathrm{~h}$. It is interesting to note that the obtained tracks were different in the various CR-39 detectors.

Atomic force microscopy was used by Yasuda et al. [80] to study surface roughness and sensitivity $\left(V_{\mathrm{t}} / V_{\mathrm{b}}\right)$ in three types of CR-39 detectors. The experiments were conducted for three types of detector, namely, pure CR-39, CR-39 doped with antioxidant and copolymer of CR-39/NIPAAm. An inverse correlation between the track sensitivity and the roughness of detector surface was found.

Palmino et al. [81] used AFM to investigate tracks in LR 115 detectors. It is interesting to note that a special bath was designed to study alpha-particle track developments during the etching in situ and in real time.
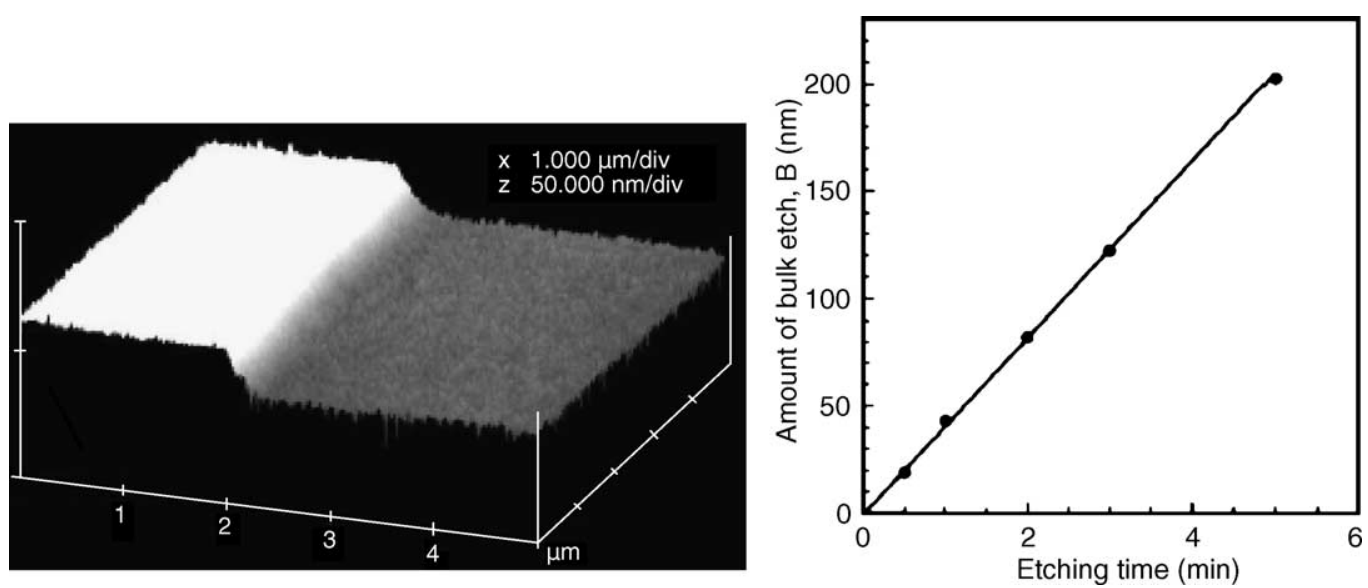

Fig. 24. Left panel: three-dimensional AFM image of a piece of CR-39 detector after etching in $7 \mathrm{~N} \mathrm{NaOH}$ at $70{ }^{\circ} \mathrm{C}$ for $5 \mathrm{~min}$ [71]. Right panel: the thickness of the removed layer as a function of the etching time [71]. 


\subsubsection{Conclusions}

As a conclusion of this section, we may emphasize that the bulk etch rate of the CR-39 detector is less influenced by the composition and the manufacturing procedure. In addition, the detector response is stable with the age. The different values that appear in the literature are caused by different etching conditions used by different authors. On the other hand, the bulk etch rate of the LR 115 detector shows significant dependence on the storage, age and other relevant factors. Irradiation of the detectors with gamma rays, or some other low LET radiation, even with non-ionizing radiation increase the bulk etch rate. Furthermore, depth dependence of the bulk etch rate is also observed in some studies, but this effect is not explained in full up to now.

\subsection{Track etch rate ( $V_{t}$ function)}

The track etch rate $V_{\mathrm{t}}$ is the rate of detector etching along the particle track, which is along the preferential direction of etching. A track is formed when $V_{\mathrm{t}}>V_{\mathrm{b}}$. For an oblique incidence of the particle, a track is formed when $\sin \theta>V_{\mathrm{b}} / V_{\mathrm{t}}$. Usually the ratio between two rates, viz. $V=V_{\mathrm{t}} / V_{\mathrm{b}}$ is used. $V$ is convenient to be expressed as a function of the residual range $R$ of the particle in the detector material, i.e., $V(R) . V$ is also called the detector sensitivity $S$, or detector response in some works. Some authors also referred to this as "calibration of a detector". Furthermore, the reduced track etch rate $(V-1)$ is also used.

In earlier days of investigation of track detectors, $V$ was considered constant. This is valid in some cases, but in general $V$ is variable along the particle trajectory. Determination of the $V$ function is rather difficult and sometimes problematic (in contrary to $V_{\mathrm{b}}$ where the determination is relatively much easier). Several methods were described in the literature for determination of the $V$ function.

According to Durrani and Bull [5], the ratio of $V_{\mathrm{t}} / V_{\mathrm{b}}$ can be determined from:

$$
V=\frac{V_{\mathrm{t}}}{V_{\mathrm{b}}}=\frac{4 V_{\mathrm{b}}^{2} t^{2}+D^{2}}{4 V_{\mathrm{b}}^{2} t^{2}-D^{2}}
$$

where $D$ is the track diameter and $t$ is the etching time. This formula (or some modifications of it) can only be used for constant $V_{\mathrm{t}}$ so it is of limited value.

Some methods are based on investigation of the track parameters. Fitting the set of data on the track parameters is one possible way to determine the $V$ function. Here, the track parameters, namely, the major and minor axes are dependent on the shape of the $V$ function (for a given etching condition and a thickness of the removed layer). The track parameters have been measured for various incident energies and angles of some particles. Computer programs incorporating a variety of $V$ functions are developed which calculate the track parameters (e.g., [82-84]), which are then compared with the experimental data set. The function which generates track parameters closest to the experimental data will be chosen. This fitting method is rather indirect and time consuming.

Another method in determination of the $V$ function is based on studies on the track profile. The biggest problem for this method is the difficulties in acquiring the track profiles. Calculation of the track profile is relatively easy, but experimental determination is rather difficult. Different experimental methods have been applied to obtain track profiles, including three-dimensional confocal microscope, irradiation of the detector from lateral side, breaking the detector, and measuring replicas of tracks, etc. A survey of different measurement methods and the reported $V$ functions are given further. 


\subsubsection{Survey of $V$ function measurements for various detectors}

Somogyi and Almasi [85] determined the $V$ function in the LR 115 detector by following the time when the thin sensitive layer was perforated by the track and extracted information about the $V$ function. They found $V$ as a function of the residual range of alpha particles in the LR 115 detector as:

$$
V=1+\mathrm{e}^{-0.205 R(\mu \mathrm{m})+3}
$$

for etching in $10 \% \mathrm{NaOH}$ at $60{ }^{\circ} \mathrm{C}$.

Somogyi and Hunyadi [86] measured $V_{\mathrm{t}}$ in the CR-39 detector using the following formula:

$$
V_{\mathrm{t}}=V_{\mathrm{b}} \frac{1+r^{2}}{1-r^{2}}
$$

where $r=d_{\alpha} / d_{\mathrm{f}}$ is the ratio of the diameter of alpha-particle tracks to fission tracks. This formula also implied a constant $V_{\mathrm{t}}$. They obtained:

$$
V=\frac{V_{\mathrm{t}}}{V_{\mathrm{b}}}=1.158 C^{1 / 4}
$$

where $C$ is concentration in units of normality. This formula was verified for $\mathrm{NaOH}$ solutions with normalities between 1 and $10 \mathrm{~N}$.

Henshaw et al. [87] determined the response of the CR-39 detector to alpha particles, and found $V_{\mathrm{t}}$ as:

$$
V_{\mathrm{t}}=k R_{\mathrm{S}}^{-n}
$$

where $R_{\mathrm{S}}$ was the residual range of an alpha particle in the detector, $n=0.2$ and 0.29 for etching temperatures of 20 and $75{ }^{\circ} \mathrm{C}$, respectively, in $6.25 \mathrm{~N} \mathrm{NaOH}$. The corresponding values for $V_{\mathrm{b}}$ are $0.0182 \pm 0.0015$ and $2.46 \pm 0.09 \mu \mathrm{m} / \mathrm{h}$ obtained from the diameter of the track openings of fission fragments from ${ }^{252} \mathrm{Cf}$.

Giacomelli et al. [88] measured $V_{\mathrm{t}} / V_{\mathrm{b}}$ as a function of restricted energy loss for samples of different ages. The CR-39 detectors were provided by Intercast Europe Co. from 1989 to 1994. Etching was performed in $6 \mathrm{~N} \mathrm{NaOH}$ solution at temperatures 70 and $40{ }^{\circ} \mathrm{C}$, and $V_{\mathrm{t}} / V_{\mathrm{b}}$ was found from Eq. (3.9). They related $V$ to the restricted energy loss, and the results are presented in Fig. 25. It can be seen that $V_{\mathrm{t}} / V_{\mathrm{b}}$ increases with REL. Slight differences in the detector response were attributed to the different ages of the detectors as well as to the experimental uncertainties due to manufacturing and etching.

Singh et al. [89] showed that $V_{\mathrm{t}} / V_{\mathrm{b}}$ of heavy-ion tracks in garnet crystals depended on the annealing temperature of the detector. With the increase in the annealing temperature from 298 to $1073 \mathrm{~K}$, the reduced $V\left(V_{\mathrm{t}} / V_{\mathrm{b}}-1\right)$ decreases from 0.82 to 0.220 .

Yamamoto et al. [90] used atomic force microscopy to study the sensitivity of CR-39 detectors to heavy ions. They determined $V_{\mathrm{t}} / V_{\mathrm{b}}$ by using Eq. (3.9). The CR-39 detectors were irradiated by a $490 \mathrm{MeV} /$ nucl $\mathrm{Si}$ beam, and $V_{\mathrm{b}}$ was determined from the etch-pit radii of fission fragments. The detectors were etched in $7 \mathrm{~N} \mathrm{NaOH}$ at $70{ }^{\circ} \mathrm{C}$ for $10 \mathrm{~min}$ and scanned by AFM. Some tracks can be found by AFM after very short etching time. From these results, the authors concluded that an etch induction time was a phenomenon related to the optical microscope and was not an essential phenomenon of track etching mechanisms. One can say here that the tracks of alpha particles may be seen after etching of only $1 \mathrm{~min}$. That is a confirmation that induction time is not a real effect.

Tracks obtained under an oblique incident angle can be used for determination of $V_{\mathrm{t}}$. Here, the major and minor axes of the track openings are used. Doke et al. [91] found the dependence of $V_{\mathrm{t}} / V_{\mathrm{b}}$ in 


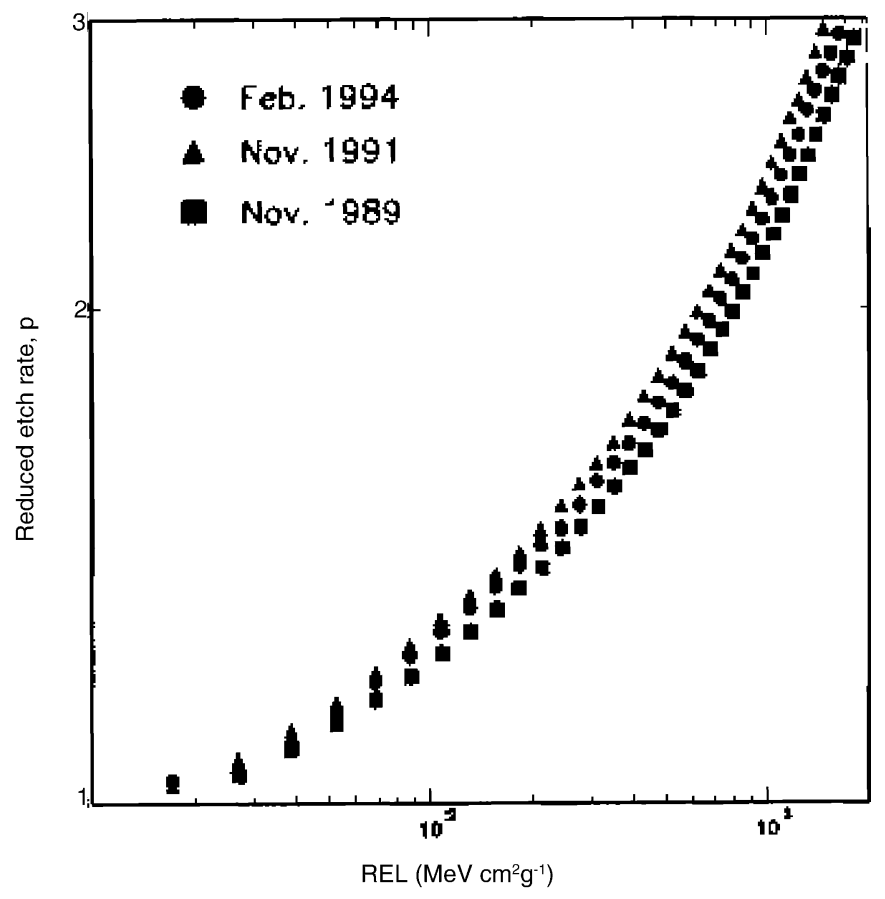

Fig. 25. Calibration results of CR-39 detectors from different years of manufacturing [88].

an antioxidant-doped CR-39 on the incident angle of ions. The sensitivity $\left(V_{\mathrm{t}} / V_{\mathrm{b}}-1\right)$ was calculated from:

$$
S=\frac{V_{\mathrm{t}}}{V_{\mathrm{b}}}-1=\sqrt{\frac{16 D_{\mathrm{A}}^{2} B}{\left(4 B^{2}-D_{\mathrm{B}}^{2}\right)^{2}}}-1
$$

where $D_{\mathrm{A}}$ and $D_{\mathrm{B}}$ were the major and minor axes of the elliptical opening and $B$ was the removed thickness of CR-39 during etching. It was found in this paper that $V$ decreased with $\sin \delta$ (where $\delta$ was the incident angle). This effect was not well explained and understood.

Meesen and Van Oostveldt [92] used confocal scanning laser microscopy to study alpha tracks in the CR-39 detector. To obtain the lateral view of the tracks, different techniques have been tested. Due to the high transparency of the CR-39 detector, direct viewing of the tracks by the refractive mode gave a relatively low contrast which was not suitable for analyses. In another method, the detector was covered by a fluorescence solution in a suitable liquid. Full three-dimensional images of the tracks were obtained. Confocal microscope was also used by Jakes et al. [93] to study the electrochemically etched tracks in the CR-39 detector.

Dorschel et al. [94] measured $V_{\mathrm{t}}$ of proton tracks in CR-39 produced in former GDR, and $V_{\mathrm{t}}$ was given as a function of the proton energy. To determine $V_{t}$, Eq. (3.9) was used. Sharp and high peaks were found at low proton energies, which were attributed to the Bragg peak in the stopping power curve. However, the positions of maxima did not correspond to the position of the Bragg peak.

Fromm et al. [52] visualized the track profile in the following way. CR-39 sheets were polished by $0.1 \mu \mathrm{m}$ granules at one edge. The polished edge was irradiated and then sequentially etched. After each stage, the chosen track was photographed. Etching was performed at $7 \mathrm{~mol} / \mathrm{l} \mathrm{NaOH}$ at $343 \mathrm{~K}$. A set of good photographs was obtained for various removed layers and incident angles of protons and alpha particles. The $V_{\mathrm{t}}$ function proposed in this paper is given further. 
An interesting approach in studying the track profile was described by Borgonovo et al. [95] who used the replica method and the transmission electron microscopy. The bulk etch rate was obtained from the diameter of ${ }^{252} \mathrm{Cf}$ fission tracks and $235 \pm 5 \AA$ /min was found for etching for $6.25 \mathrm{~N} \mathrm{NaOH}$ at $70{ }^{\circ} \mathrm{C}$ (or $1.41 \mu \mathrm{m} / \mathrm{h}$ ). Different solutions were tested for removing of CR-39 without altering the replicas. The best solution PEW was proposed as $15 \% \mathrm{KOH}, 40 \%$ ethyl alcohol and $45 \%$ distilled water at room temperature. Replicas of normally incident deuterons and grazing incident ${ }^{19} \mathrm{~F}$ ions were presented.

Yu et al. [96] proposed to use surface profilometry to determine the track lengths in CR-39 detectors through measurements of their replicas. Tracks from alpha particles with an incident energy of $4 \mathrm{MeV}$ were chosen to demonstrate the method. After irradiation and chemical etching, resin replicas were made from the tracks. The heights of the replicas were conveniently determined from the lateral view of the replicas generated by the Form Talysurf PGI profilometer.

Dorschel et al. [97] irradiated PATRAS CR-39 detectors by alpha particles perpendicularly on the surface with different energies. The detectors were chemically etched in $7.25 \mathrm{~N} \mathrm{NaOH}$ at $70{ }^{\circ} \mathrm{C}$. The detectors were then broken perpendicularly on the surface in order to reveal the track profiles. Images of the tracks from the lateral side were obtained. From these studies, they determined $V_{\mathrm{t}}$ of alpha particles in CR-39 and the results are given in Fig. 26.

From Fig. 26, we can see that the shape of the curves vary with the initial energies of alpha particles. This result is not expected if $V_{\mathrm{t}}$ is related only to the residual range of alpha particles in the detector material. This effect was later called the "depth dependence" of $V_{\mathrm{t}}$, although the dependency can be on the initial particle energy. It has been shown later that the effect is more pronounced for heavier ions.

The irradiation condition also affects the detector response. For example, deficiency of oxygen during irradiation diminishes the detector response. This was experimentally proved by Fujii et al. [98]. These results may be important for irradiation in vacuum or when the air pressure is low.

The effects of the temperature during irradiation on the detector sensitivity were recognized by Diwan et al. [99]. CR-39 (DOP) and SR-90 polymer plastics were irradiated to alpha particles at different environmental temperatures, and the track diameter decreased with the temperature. The effect was more pronounced in CR-39 than in SR-90. Since the bulk etch rate was not changed, the authors concluded that the registration temperature influenced only on the track etch rate $V_{\mathrm{t}}$.

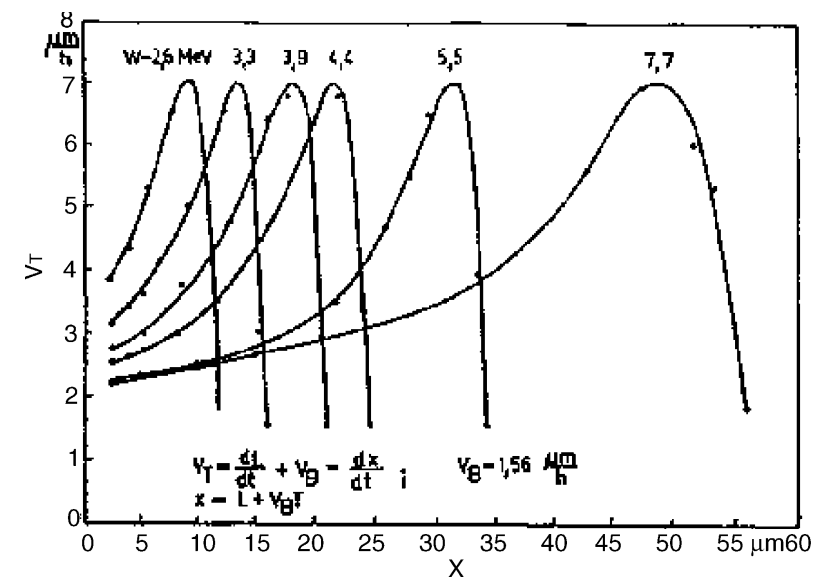

Fig. 26. Track etch rate of alpha particles in the CR-39 detector as a function of the detector depth for different incident energies [97]. 
The activation energy was also introduced for studies on the etching of the tracks, which was discussed by Awad and El-Samman [53]. The reduced track etch rate was determined from the Eq. (3.9) for circular tracks, and

$$
V=\sqrt{1+\frac{4(a / h)^{2}}{[1-(b / h)]^{2}}}
$$

for elliptical tracks, where $h$ was the removed layer, $r$ was the diameter of circular track openings, and $a$ and $b$ were the semi major and minor axes, respectively. The Arhenius type of equation was also used for the track etch rate:

$$
V_{\mathrm{t}}=B_{2} \mathrm{e}^{-E_{\mathrm{t}} / k T}
$$

where $E_{\mathrm{t}}$ was the track activation energy and $B_{2}$ was a fitting constant. $E_{\mathrm{t}}$ was also determined as 0.75 $\pm 0.03 \mathrm{eV}$, which was almost independent of the LET of incident particles.

Kumar and Prasad [55] measured $V_{\mathrm{t}}$ in different Makrofol polycarbonates irradiated by fast ${ }^{40} \mathrm{Ar}$ ions. Etching was performed in $6.25 \mathrm{~N} \mathrm{KOH}$ at $55,60,65$ and $70{ }^{\circ} \mathrm{C}$. It was also shown that $V_{\mathrm{t}}$ followed the Arhenius law of the type of Eq. (3.16). The track activation energy was found to be slightly different in various types of Makrofol detectors, viz. $0.66 \pm 0.04 \mathrm{eV}$ for Makrofol KG, $0.66 \pm$ $0.04 \mathrm{eV}$ for Makrofol KL and $0.66 \pm 0.04 \mathrm{eV}$ for Makrofol $\mathrm{N}$ detectors.

The CR-39 detector response in terms of $V_{\mathrm{t}} / V_{\mathrm{b}}$ for light ions was measured by Yamauchi et al. [100]. The CR-39 detectors manufactured by the Baryotrack Fukuvi Chemical Co. was irradiated by protons, deuterons, and ${ }^{7} \mathrm{Li}$ ions with different energies. The detectors were etched in multiple steps and the diameters of the tracks were measured, from which $V_{\mathrm{t}} / V_{\mathrm{b}}$ was deduced. The obtained results are given graphically in Fig. 27.

Cecchini et al. [56] irradiated Intercast CR-39 detectors with ions of ${ }^{82} \mathrm{~Pb}^{+}$and ${ }^{26} \mathrm{Fe}^{+}$in order to study the effects of aging and fading. The detectors were intended to be irradiated for 4 years in the Gran Saso Laboratory in searching for magnet monopoles. $V_{\mathrm{t}} / V_{\mathrm{b}}=p$ was found from Eq. (3.9), and $p$ was expressed as a function of REL 200 in Fig. 28.

The variations in the $V_{\mathrm{t}} / V_{\mathrm{b}}$ curves for the detectors of different ages were explained by the variations in $V_{\mathrm{b}}$, and the conclusion was that the CR-39 detector response was stable for more than 10 years, with fading below $10 \%$ and the detector fully sensitive after 12 years.

The confocal microscope also enables recording of the track profile. Explanation of operation principles and comparisons among different scanning microscopes in track studies (including confocal microscopes, scanning tunneling microscopes, scanning force microscopes) were given by Vukovic

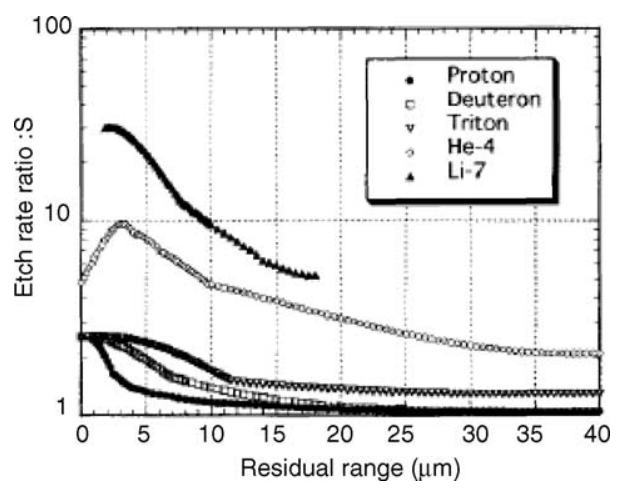

Fig. 27. $S=V_{\mathrm{t}} / V_{\mathrm{b}}$ in CR-39 for different ions derived from the growth of etch-pit diameters [100]. 


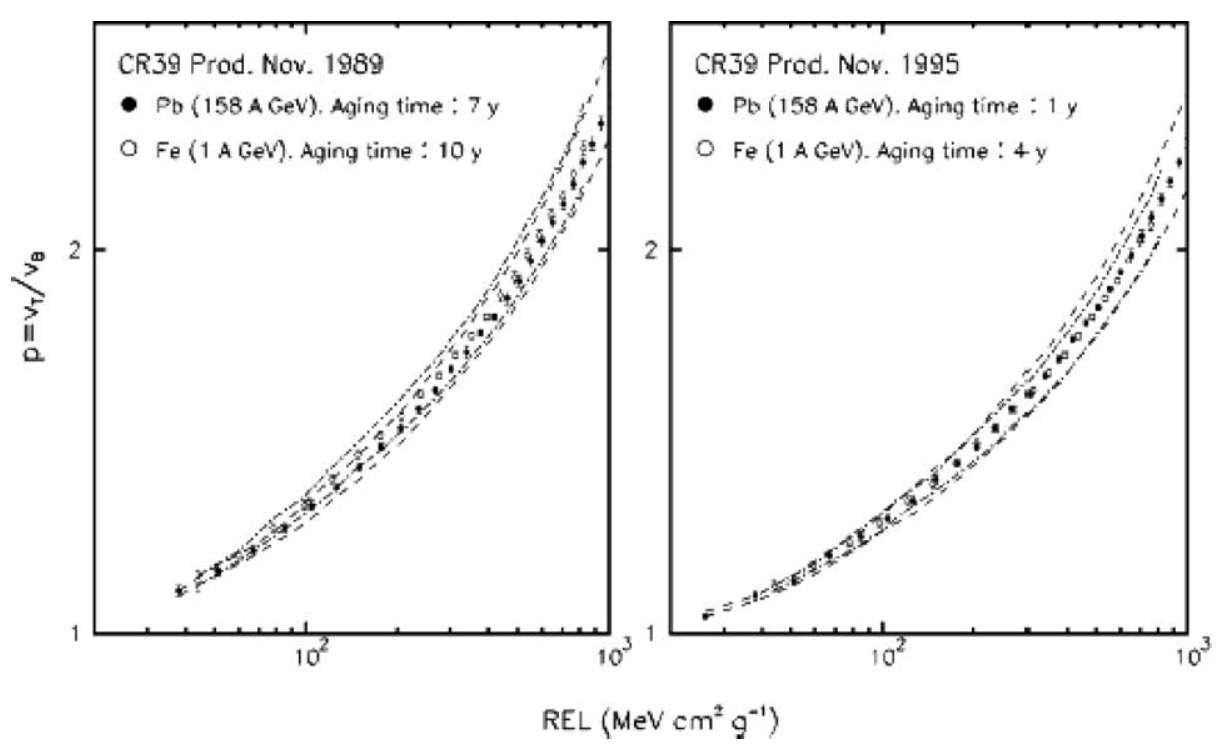

Fig. 28. $V_{\mathrm{t}} / V_{\mathrm{b}}$ curves for $\mathrm{Pb}$ and $\mathrm{Fe}$ ions for detectors of different ages, as a function of restricted energy loss [56].

and Atanasijevic [76]. Confocal microscopy was used by Gais et al. [101] to study alpha tracks originated from radon and its progeny in the CR-39 detector.

Vaginay et al. [102] used confocal three-dimensional microscope to determine the sensitivity $\left(V_{\mathrm{t}} /\right.$ $V_{\mathrm{b}}$ ) of the Baryotrak CR-39 detector. The detectors were irradiated with ${ }^{7} \mathrm{Li}$ ions and etched in $7.25 \mathrm{~N}$ $\mathrm{NaOH}$ solution at $70{ }^{\circ} \mathrm{C}$. After etching, the samples were kept in a fluorescence solution, and the confocal microscope was then used to obtain three-dimensional images of the tracks. Confocal images of Li tracks are given in Fig. 29.

Results for $V_{\mathrm{t}}$ obtained with confocal microscopy were compared with optical results. The curves obtained with these two methods agree with each other, but the confocal microscope method gives larger $V_{\mathrm{t}}$ in the region of the Bragg peak.

Computer analytical techniques which automated the extraction of track parameters from images obtained by means of three-dimensional confocal microscope were described by Meesen and Poffijn [103]. The track lengths and track diameters obtained by using automatic three-dimensional track analyses were compared to manual measurements and good agreement was found.

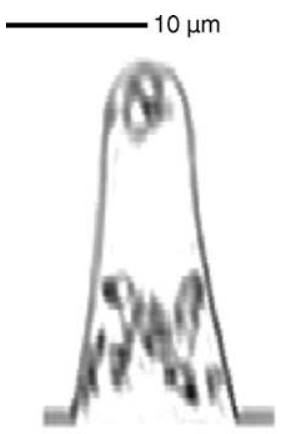

(a)

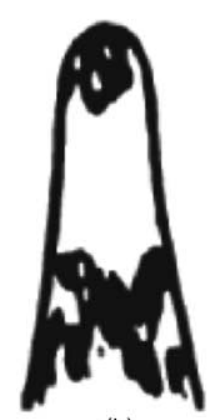

(b)

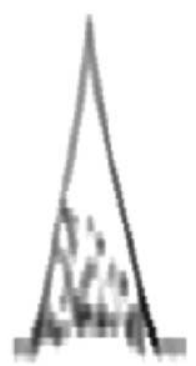

(c)

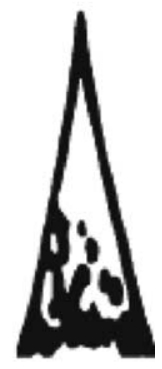

(d)

Fig. 29. Confocal microscopic images of normally incident Li tracks in Baryotrak CR-39 detectors [102]. (a and b) $10.77 \mathrm{MeV}$ with $5 \mathrm{~h}$ and 30 min etching; (c and d) $10.77 \mathrm{MeV}$ with $3 \mathrm{~h}$ and 30 min etching, (a) and (c) are rough images while (b) and (d) are smoothed and binarized images. 


\subsubsection{Examples of some $V_{t}$ functions for alpha tracks}

Some examples of the $V$ function for the special case of alpha particles for the CR-39 detector, which is important in radon and progeny dosimetry, are given further. In [5], the $V$ function for alpha particles in CR-39 (originally from [38]) is given as:

$$
V=1+\left(a_{1 \mathrm{G}} \mathrm{e}^{-a_{2 \mathrm{G}} R^{\prime}}+a_{3 \mathrm{G}} \mathrm{e}^{-a_{4 \mathrm{G}} R^{\prime}}\right)\left(1-\mathrm{e}^{-a_{5 \mathrm{G}} R^{\prime}}\right)
$$

with the constants $a_{1 \mathrm{G}}=11.45, a_{2 \mathrm{G}}=0.339, a_{3 \mathrm{G}}=4, a_{4 \mathrm{G}}=0.044$ and $a_{5 \mathrm{G}}=0.58$. In [52] mentioned earlier, the following function was given:

$$
V_{\mathrm{t}}=V_{\mathrm{b}}+\frac{a_{1}}{a_{2}^{2}+\left[a_{3}(L-x)-\left(1 / a_{4}(L-x)\right)\right]^{2}}
$$

The $V_{\mathrm{t}}$ function was empirically proposed in this form and the constants were determined by fitting as $a_{1}=0.53, a_{2}=0.80, a_{3}=0.04$ and $a_{4}=0.12$.

Another example of the $V$ function for alpha particles in CR-39 was given by Brun et al. [104] in the form:

$$
V=1+\mathrm{e}^{-a_{1} R^{\prime}+a_{4 \mathrm{~B}}}-\mathrm{e}^{-a_{2 \mathrm{~B}} R^{\prime}+a_{3}}+\mathrm{e}^{a_{3 \mathrm{~B}}}-\mathrm{e}^{a_{4 \mathrm{~B}}}
$$

with the constants $a_{1 \mathrm{~B}}=0.1, a_{2 \mathrm{~B}}=1, a_{3 \mathrm{~B}}=1.27$ and $a_{4 \mathrm{~B}}=1$. The two functions given in Eqs. (3.17) and (3.19) are shown in Fig. 30. Both functions show maxima close to the end of the particle range, corresponding to the Bragg peak in the stopping power curves. However, these two functions are significantly different particularly in the low-energy region (Bragg peak).

The $V$ function of alpha particles in the LR 115 detector was presented by Durrani and Bull [5] in the form:

$$
V=1+\left(100 \mathrm{e}^{-0.446 R}+5 \mathrm{e}^{-0.107 R}\right)\left(1-\mathrm{e}^{-R}\right)
$$

where $R$ was the residual range. Comparisons between the $V$ functions for the LR 115 and CR-39 detectors reveal that the $V$ function is much larger for the LR 115 detector. The difference is particularly pronounced in the low-energy region close to the end of the particle path.

\subsubsection{Discussions about $V_{t}$}

The $V$ function has a maximum close to the end of the particle range, which is evident from Fig. 30. The shape is similar to the Bragg ionizing curve. In some ways, the $V$ function is a reflection of

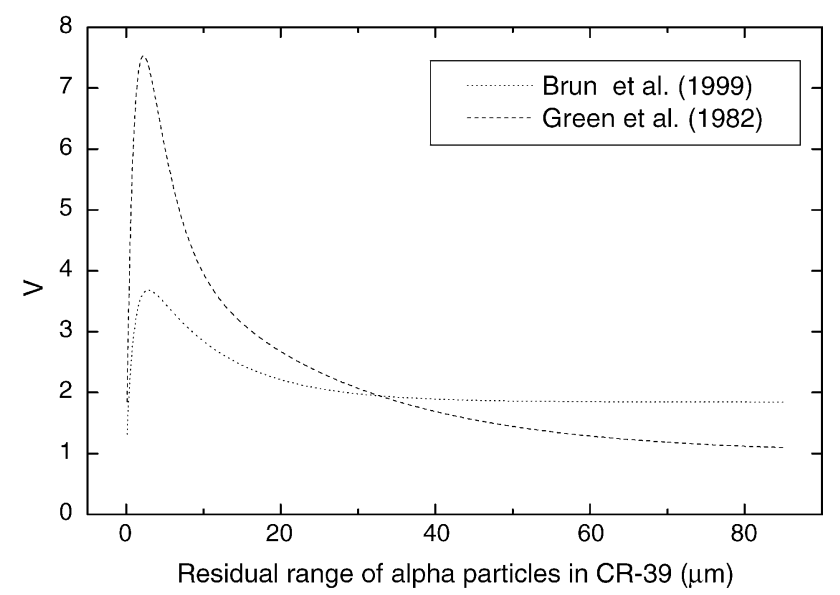

Fig. 30. The $V$ functions of alpha particles track in CR-39 according to Brun et al. [104] and Green et al. [38]. 
some parameters for primary interaction of the particle with the detector material. Some authors applied the $V$ function in the form of $V=a R^{\prime-b}$ where $a$ and $b$ are constants. Such a function can create problems in calculations because $V$ will become too large when $R^{\prime}$ becomes very small. Functions of this form have no maximum and do not represent realistic situations. It is even farther away from reality than the assumption of a constant $V$.

The most frequently used $V$ function is related to the restricted energy loss (e.g., Fig. 28). The argument against the relation between $V$ and the total energy loss (or stopping power) is that some $\delta$ electrons formed in the interactions between heavy ions and the detector material can be too energetic and will bring their energies too far away from the particle track. In this way, they will cause damages to the detector structure which are not correlated with the particle track and which do not take part in the track formation. There were discussions about the cutoff energy for $\delta$ electrons and Henshaw et al. [105] proposed $200 \mathrm{keV}$ as the cutoff energy.

Baiocchi et al. [106] irradiated Intercast CR-39 detectors with different kinds of heavy ions. They compared the reduced $V$ function and restricted energy loss, and concluded that a single curve between them is enough to describe the relationship.

There are several formulas that relate $V$ with REL. For example, Petersen and Enge [107] published the following expressions for the Makrofol detectors:

$$
\frac{V_{\mathrm{t}}}{V_{\mathrm{b}}}-1=0.0035 \mathrm{REL}^{3.97}
$$

for $8 \mu \mathrm{m}$ Makrofol $\mathrm{KG}$ etched at $50{ }^{\circ} \mathrm{C}$, or

$$
\frac{V_{\mathrm{t}}}{V_{\mathrm{b}}}-1=0.00393 \mathrm{REL}^{2.99}
$$

for $8 \mu \mathrm{m}$ Makrofol $\mathrm{KG}$ etched at room temperature.

Dorschel et al. [108] concluded that unique functions between $V$ and REL can be determined for protons, deuterons and alpha particles. $V$ was determined from the recalculations of the etch-pit evolution, and the relationships were:

$$
\begin{aligned}
V_{\text {mean }}= & 0.93+3.14 \times 10^{-3} \mathrm{REL}-7.80 \times 10^{-6} \mathrm{REL}^{2}+1.11 \times 10^{-8} \mathrm{REL}^{3}-5.27 \\
& \times 10^{-12} \mathrm{REL}^{4}
\end{aligned}
$$

for $33 \mathrm{MeV} / \mathrm{cm} \leq \mathrm{REL} \leq 560 \mathrm{MeV} / \mathrm{cm}$ and

$$
V_{\text {mean }}=1.30+3.80 \times 10^{-4} \mathrm{REL}+4.90 \times 10^{-7} \mathrm{REL}^{2}
$$

for $560 \mathrm{MeV} / \mathrm{cm} \leq \mathrm{REL}$, where REL has the unit $\mathrm{MeV} / \mathrm{cm}$.

It was assumed and strongly believed that $V_{\mathrm{t}}$ can be successfully represented as a function only of the residual range or only of the restricted energy loss. This is correct for light ions like protons, deuterons and alpha particles. However, Dorschel et al. [109] recently found that the $V_{\mathrm{t}}$ functions of some ions in CR-39, like ${ }^{7} \mathrm{Li},{ }^{11} \mathrm{~B}$ and ${ }^{12} \mathrm{C}$, are dependent not only on REL, but also on the depth within the detector where this energy loss occurs. Arranging the etch rate ratio as a function of two variables, REL and $x$, all experimental results were explained without inconsistencies. The authors referred to this as the "depth dependence" of $V_{\mathrm{t}}$. In fact, the maximum of the $V$ function has been experimentally found to depend on the initial energy of the light ion. This work was later extended to heavier ions like ${ }^{14} \mathrm{~N}$ and ${ }^{16} \mathrm{O}$ [110]. The height of the maximum of the $V$ function close to the end of the particle range depends on the initial particle energy, which is illustrated in Fig. 31. The height of the maximum increases with the initial energy. Explanations to this effect have not yet been provided until now. 

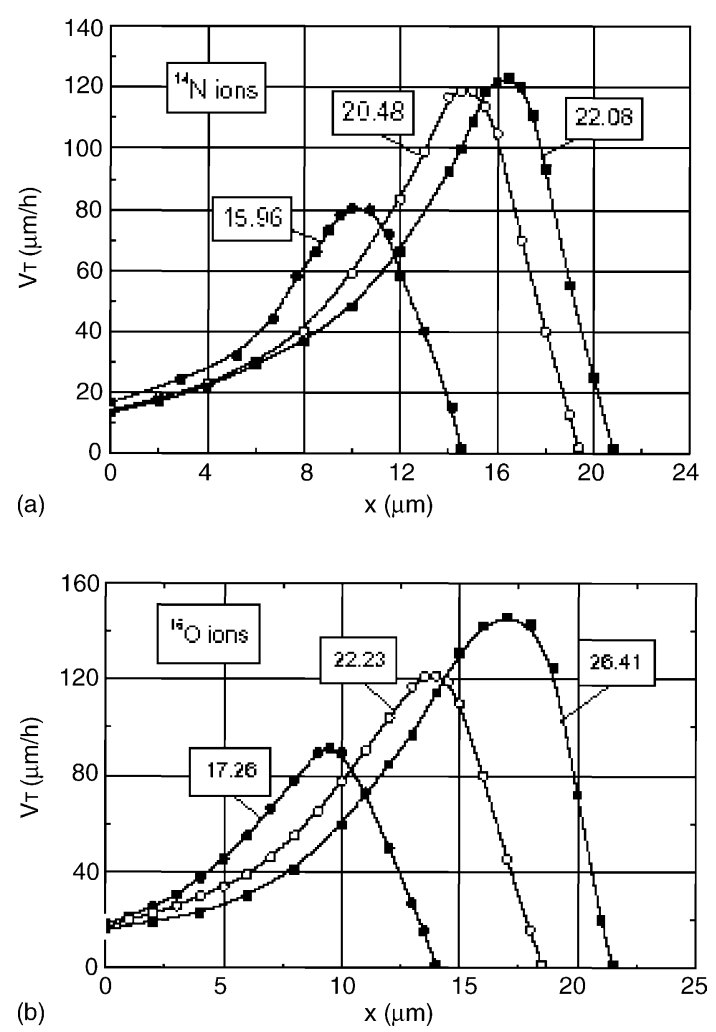

Fig. 31. The track-etch rate as a function of the depth $x$ within the detector for various initial ion energies given on the curves in $\mathrm{MeV}$ for: (a) ${ }^{14} \mathrm{~N}$ ions; (b) ${ }^{16} \mathrm{O}$ ions [110].

In [111], a correlation between $V$ and several parameters of the primary-particle interaction was sought. The studied parameters included the linear energy transfer LET, the restricted energy loss $\mathrm{REL}_{150}$, ionising rate and radial delta ray dose. Investigations were performed for protons, alpha particles, lithium and carbon ions. No good correlation of $V_{\mathrm{t}}$ was found with any of the investigated parameters. The peak in the $V_{\mathrm{t}}$ function appeared at different places in comparison to the peaks in REL, LET, dose and ionising rate curves. In addition, the shape of the $V_{\mathrm{t}}$ curves is different in comparison to that of the curves of other investigated parameters.

Failure to establish good correlations among the primary-particle interactions and the $V$ function as well as the difficulties in accurately predict the values and behavior of this function have indicated that the track-etching process is not yet fully understood.

\section{Application of track detectors for radon measurements}

\subsection{Radon measurements with track detectors}

As was described in previous chapters, after passage of an alpha particle through a dielectric material, a latent track is formed. After chemical treatment, the track can be visualized and can be seen under an optical microscope. This is the basic principle of application of track detectors for radon measurements. Radon and some of its short-lived progeny emit alpha particles. In the radioactive 
decay chain of ${ }^{222} \mathrm{Rn}$, three groups of alpha particles are emitted, namely, the alpha particles with energies of $5.49 \mathrm{MeV}$ emitted from ${ }^{222} \mathrm{Rn}$, those with energies of $6 \mathrm{MeV}$ from ${ }^{218} \mathrm{Po}$ and those with the most energetic $7.69 \mathrm{MeV}$ from ${ }^{214} \mathrm{Po}$. Other radon isotopes, such as ${ }^{220} \mathrm{Rn}$ (often referred to as thoron) can also be present in air and emit alpha particles that may be detected by track detectors.

Track detectors are used for radon measurements for different purposes. Radon is measured in the atmosphere, soil or water. Measurements of radon in underground water and deep wells are used for earthquake prediction or for studying active faults. Radon emanation and exhalation from building materials are also measured with track detectors. Radon entries from soil into buildings and houses are also investigated by track detectors. Separate measurements of radon and thoron in air or soil are also possible using track detectors.

Track detectors are applied for radon measurements in air in two basic modes. A recent review of application of track detectors for radon measurements has been given by Nikolaev and Ilic [112]. The first mode employs "open" or bare detectors where the detectors are simply immersed in the air at the location where the measurements are conducted. Alpha particles emitted by ${ }^{222} \mathrm{Rn}$ and its progeny strike the detectors and leave latent tracks in it. The exposure time $t$ is known and depends on the radon and progeny concentrations as well as the desired uncertainties of the measurements. After exposure of the detectors, they are returned to the laboratory for etching and subsequent track analyses. The main measured quantity is track density per unit exposure time, $\rho$, expressed in track $/\left(\mathrm{m}^{2} \mathrm{~s}\right)$. The average radon concentration $C$ during the exposure time is calculated from:

$$
C=k \rho
$$

where $k$ is the calibration coefficient. Since the radon concentration is expressed with the unit $\mathrm{Bq} / \mathrm{m}^{3}$, the calibration coefficient is given in the unit $\mathrm{m}^{-1}$, because $\mathrm{Bq} \mathrm{s}$ is dimensionless. Very often the calibration coefficient is expressed in $\left(\mathrm{Bq}\right.$ day $\left./ \mathrm{m}^{3}\right) /\left(\operatorname{track} / \mathrm{cm}^{2}\right)$ or $\left(\mathrm{kBq} \mathrm{h} / \mathrm{m}^{3}\right) /\left(\operatorname{track} / \mathrm{cm}^{2}\right)$. This opendetector technique for radon measurements was very widely employed in the past. However, this mode has some disadvantages which are described further. Nevertheless, this mode is still being deployed because it is simple and inexpensive.

In addition to ${ }^{222} \mathrm{Rn}$ and its progeny, many different species like dust particles, water vapor, etc. are also present in the air. During irradiation, the particles can set down onto the detector and water vapor can condensate and create a thin film of water on the detector. The dust particles and water condensation can affect the detection of alpha particles emitted in air by absorbing or decreasing their energies.

The second problem is more fundamental than the previous one, namely, ${ }^{222} \mathrm{Rn}$ in air is always accompanied by its short-lived progeny that are also alpha emitters. As the track detectors register alpha particles from radon itself as well as those from its progeny, this technique measures both radon and its progeny. The ratio between the progeny concentrations and the radon concentration, which is surrogated by the so-called equilibrium factor, changes with meteorological and other environmental conditions. In this way, even if the calibration has been performed in an atmosphere with a known equilibrium factor, the derived results can be wrong because there cannot be guarantee of the same equilibrium factor during the real irradiation.

The third problem in radon measurements with open detectors is the presence of ${ }^{220} \mathrm{Rn}$ and its progeny in air. Although the concentration of ${ }^{220} \mathrm{Rn}$ is usually small, and on average amounts to about only $5 \%$ of the ${ }^{222} \mathrm{Rn}$ concentration, under some circumstances the ${ }^{220} \mathrm{Rn}$ concentrations may be much larger. An example can be found in buildings in Hong Kong [113-115], where high concentration of ${ }^{220} \mathrm{Rn}$ can be found due to the relatively high concentrations of ${ }^{232} \mathrm{Th}$ in the building materials [116]. The presence of ${ }^{220} \mathrm{Rn}$ disturbs the measurements of ${ }^{222} \mathrm{Rn}$ and may cause an overestimation of the derived ${ }^{222} \mathrm{Rn}$ concentrations. 


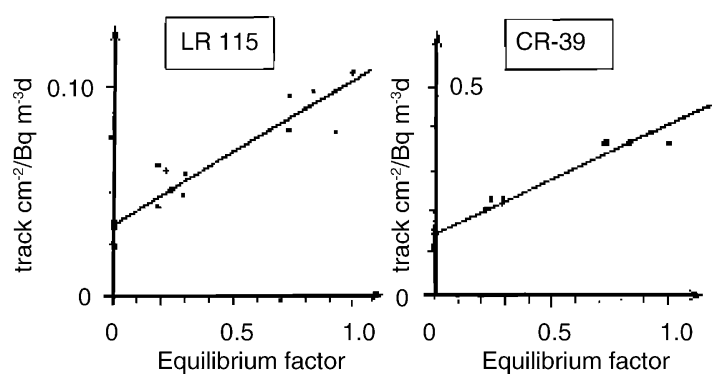

Fig. 32. Calibration coefficients for open LR 115 and CR-39 detectors for radon measurements as a function of the equilibrium factor between radon and its progeny [117].

A particular problem in radon measurements with open detectors arise when the upper energy limit of the detector is larger than the initial energy of alpha particles from the radon progeny, which is the case for CR-39 detectors. It is well known that radon progeny can be deposited onto detector surfaces; this process of progeny deposition is called "plate-out". For the case of CR-39 detectors, the alpha particles emitted from the plate-out radon progeny can form alpha tracks, with efficiencies even larger than those alpha particles emitted from radon progeny in air. The detection probability can be as high as $40 \%$. Since the amount of plate-out radon progeny changes with time and location and cannot be predicted accurately, the accuracy of radon measurements will be affected significantly.

Despite the disadvantages of the open-detector method mentioned earlier, the technique should not be dismissed completely. The open detectors carry information about the concentrations of radon and its progeny in air. The question is how to extract these concentrations separately in a reliable and accurate way. This problem will be addressed later in more detail. However, for radon measurements only, this method is not very reliable.

Calibrations of open LR 115 and CR-39 detectors for radon measurements were performed by Liehu et al. [117]. The calibration coefficients were given as a function of the equilibrium factor in Fig. 32.

A number of interesting features can be observed in Fig. 32. The first one is that the sensitivity of the CR-39 detector is about four times larger than that of the LR 115 detector. However, it is known that there is a contribution from the plate-out progeny to track formation in the CR-39 detector. The second one is the relatively larger scattering of experimental data for the LR 115 detector. Finally, the calibration coefficients increase linearly with the equilibrium factor for both detectors.

\subsection{Radon measurements with closed detectors}

In order to overcome the disadvantages of the open detector as mentioned earlier, closed detectors are used. The detector is now inserted into a cup with the open end covered by some permeable materials like a filter paper. This is sometimes called the "cup technique". The filter is permeable to radon so it can penetrate into the chamber by diffusion. This is also the reason why this device is sometimes called the "diffusion chamber". Radon progeny, dust and water vapor are stopped on the filter. In this way, some of the major problems with the use of open detectors are overcome. However, by using the concept just described, the presence of ${ }^{220} \mathrm{Rn}$ will still disturb the measurements of ${ }^{222} \mathrm{Rn}$ and may cause an overestimation of the derived ${ }^{222} \mathrm{Rn}$ concentrations [118-120]. Different types of chambers are being used by different authors. One commonly used design is shown in Fig. 33.

The detectors are usually positioned on the bottom of the chamber, but other positions are also used in practice. The center of the detectors matches the center of the base of the chamber. Some 


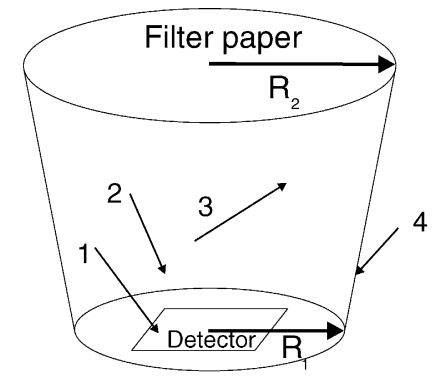

Fig. 33. A schematic diagram showing a conical diffusion chamber used for radon measurements. $R_{1}$ and $R_{2}$ are radii of the cone bases.

designs include small stands for the detector on the bottom. The chamber presented in Fig. 33 is conical. If $R_{1}=R_{2}$, the chamber is cylindrical and such chambers are also often used.

A review of different designs of diffusion chambers described in the literature is given by Nikolaev and Ilic [112], and no review is given here again. Regarding the shape, the diffusion chambers can be cylindrical, rectangular, conical or hemispherical. The detectors are positioned at different places inside the chamber and some chambers are equipped with more than one detector. Sometimes a design is used where one detector is inside the chamber (to measure only radon) and the other is outside (to measure radon + progeny).

Since 1999, not many new designs of the diffusion chamber have been described in the literature. Here, we would give only a few examples of new designs. One of them has been published by Sciocchettia et al. [121], which is schematically presented in Fig. 34.

Here, the piston is moveable and serves to switch on and off the device at the monitoring sites to permit accurate measurements of timed exposures. This integrating radon dosimeter is specially designed for radon measurements and detection at all kinds of working places at risk. Two positions were possible for the CR-39 detector, one on the filter and another on the pistol itself. By moving the pistol, the effective volume in front of the detector and thus the detector efficiency are changed and adapted to the real field situation. A twin chamber intended for radon and thoron measurements is also presented.

Another example of new chamber design, called RADOMAN, proposed by Csige and Csegzi [122] is shown in Fig. 35. An interesting feature in this geometry is that the CR-39 detector can be exposed from both sides, for which the upper surface of the detector "sees" a much larger volume of

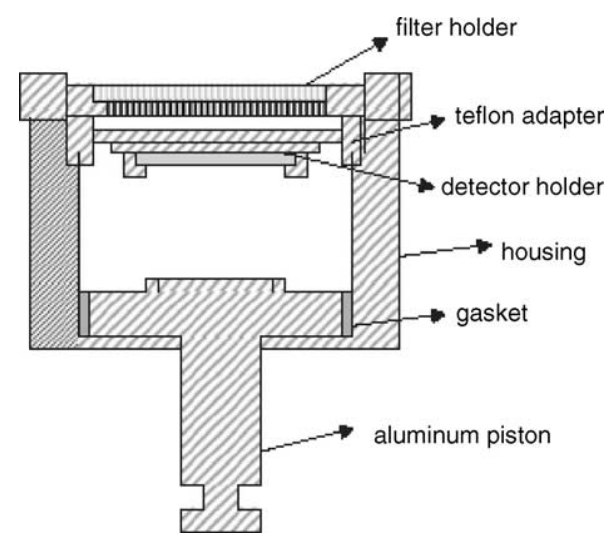

Fig. 34. Radon piston dosimeter [121]. 


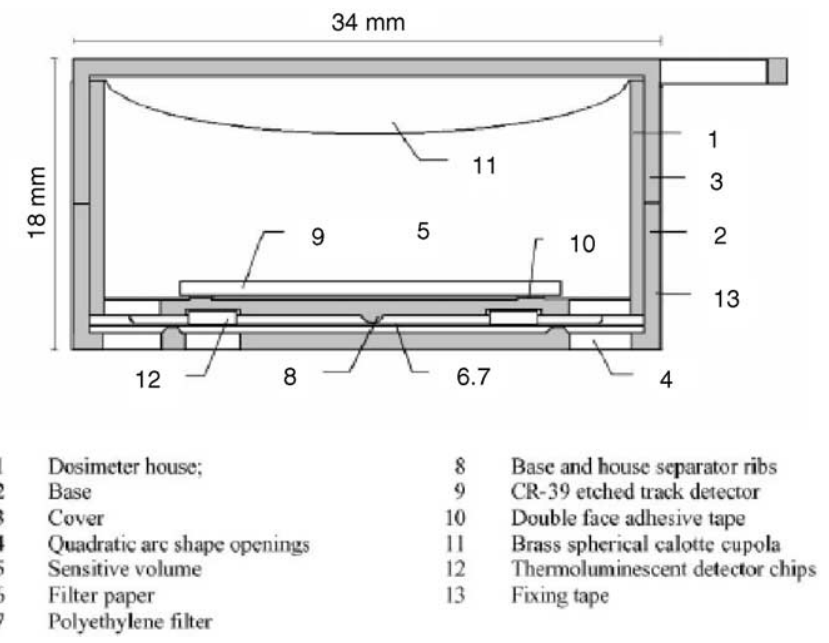

Fig. 35. Schematic diagram of the RADOMAN etched-track type radon detector [122].

air and the number of tracks is about 15 times larger than that in the lower side of the detector. A spherical calotte cupola (no. 11 in Fig. 35) is facing the detector at the top of the dosimeter house. This cupola is aimed at resulting in a uniform etched track density all over the etched track detector surface area, which is used for counting. This eliminates one source of uncertainty that may be present in such a small-volume radon detector. The radius of the sphere was optimized by Monte Carlo simulations and was also verified with experimental measurements. In the two experiments described, the track density was uniform within $4 \%$ (which was attributed to the track counting statistical error) over a $2 \mathrm{~cm}$ distance across the CR-39 detector surface.

A radon-only measuring device described by Neman et al. [123] (shown in Fig. 36) consists of two parallel acrylic plates. The detector is placed at the center of the assembly. The radon progeny cannot reach the detector because they will deposit on the internal surface of the plates, so the tracks in the internal detector are only due to alpha particles emitted by radon.

The authors claimed that the response of the detector in such an arrangement is independent of the radon progeny in the ambient air. This is not a "closed" detector technique but it is a radon-measuring device. However, the small distance between two plates of $4 \mathrm{~mm}$ will reduce the detector efficiency significantly so this setup will be most useful for measurements of high radon concentrations.

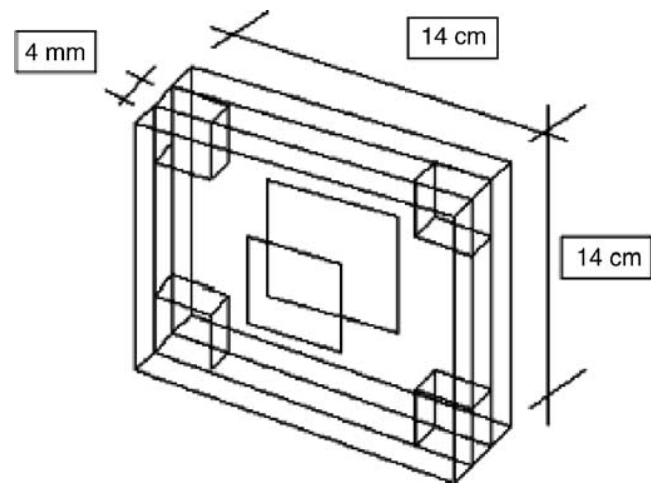

Fig. 36. Two parallel acrylic plates with a size $14 \mathrm{~cm} \times 14 \mathrm{~cm}$ with a detector at the center and the other detector on the outside surface of the plates [123]. The plates are separated by a distance of $4 \mathrm{~mm}$. 


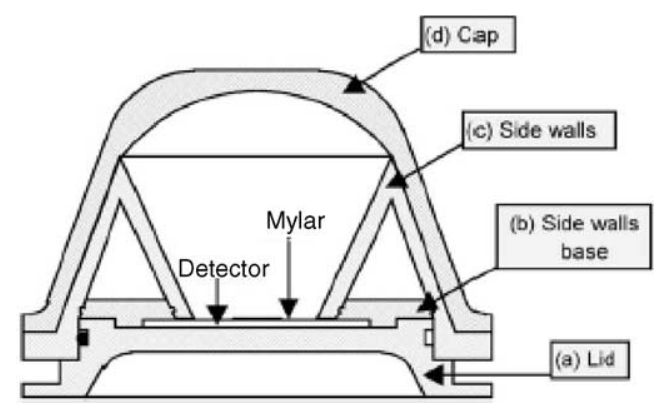

Fig. 37. The diffusion chamber proposed by Calamosca et al. [124].

Calamosca et al. [124] also presented the new design of a diffusion chamber that used the CR-39 detector as the registration medium. The dosimeter is shown in Fig. 37.

A series of Monte Carlo simulations were performed to study the system response as a function of the geometry, volume and surface, in order to optimize the design. Rotation of the spherical cap enables the closed or open operation mode of the detector. Furthermore, the Mylar film covering the detector serves to decrease the energy of alpha particles.

From this short review of new designs, one can conclude that the main stream in construction is to enable multitasking, i.e., for measuring radon and thoron simultaneously, working in closed and open modes, as well as to improve the detection characteristics and to optimize the performance.

\subsubsection{Analysis of radon measurements with closed detectors}

After the radon gas diffuses into the diffusion chamber, it can decay inside it and form new ${ }^{218} \mathrm{Po}$ atoms. These can decay further by emitting alpha particles and form other progeny in the decay chain. In Fig. 33, four possibilities are depicted. Alpha particle (1) is emitted towards the detector and the distance is short enough so it can reach the detector. This particle will leave a latent track in the detector. Whether this track can be visualized in the end depends on the incident energy, density and characteristics of damages, etching conditions, etc. Alpha particle (2) is emitted towards the detector, but the emission point is too far away from the detector; so the particle will lose all its energy in air before hitting the detector. Alpha particle (3) is not emitted towards the detector so it will not be detected as well. Alpha particle (4) is emitted towards the detector but outside the chamber. The chamber wall will prevent the detection of this particle. In the case where the chamber does not exist, alpha particle (4) would have a chance to be detected. In other words, the chamber wall prevents the detection of some alpha particles.

Inside the chamber, radioactive equilibrium between radon and its progeny is established. It seems that measurements independent of the equilibrium factor is possible with closed detectors. However, radon progeny formed inside the chamber can diffuse and deposit onto inner chamber wall. Such deposition changes the irradiation geometry and affects the detector efficiency. The first radon progeny, ${ }^{218} \mathrm{Po}$, has a relatively short half-life of $3.05 \mathrm{~min}$ and can partially decay in air and partially deposit onto the chamber wall. However, the partition is not known. The second alpha emitter in the radon decay chain, ${ }^{214} \mathrm{Po}$, is in radioactive equilibrium with ${ }^{214} \mathrm{Bi}$ that has a much longer half-life, so ${ }^{214} \mathrm{Po}$ is most likely deposited before decaying. Therefore, the whole picture of decays inside the diffusion chamber is as follows. The ${ }^{222} \mathrm{Rn}$ gas diffuses into the chamber and decays completely in air, ${ }^{218}$ Po decays partially in air and partially after deposition, while ${ }^{214}$ Po decays entirely after deposition.

The unknown fraction of deposition for ${ }^{218}$ Po introduces an uncertainty in radon measurements [125-127], which might not be smaller than that due to the unknown equilibrium factor in radon 
measurements with the open detector. In addition, it is also not known whether the deposition on the inner chamber wall is uniform. Non-uniform deposition can further complicate the analyses of the detector response in the diffusion chamber.

To minimize the effects of progeny deposition, metallic chambers are used instead of the plastic chambers. Some authors used to cover the inner surface with a conductive layer to reduce the deposition by eliminating static electricity. However, electrostatic deposition is not the only mechanism for progeny deposition. Other mechanisms like diffusion and gravitational deposition can be important, and the problem due to progeny deposition is not solved completely.

From this short analysis, it is noticed that the detector response, expressed in the track number per unit surface area in unit time, depends on the dimension and shape of the chamber, the dimension, location and shape of the detector, as well as the etching and readout procedures. If one can design a chamber in such a way that the detection efficiency of radon progeny in air is equal to the detection efficiency of radon progeny on the chamber wall, the uncertainty introduced by the unknown fraction of ${ }^{218}$ Po deposition can be eliminated.

The effect of plate-out is important in diffusion chambers. Radon progeny might deposit onto the internal wall of the chamber including the filter as well as the detector itself. The detection probability for alpha particles emitted just on the surface of the detector is much larger than that for alpha particles emitted in air. The LR 115 detector has an upper energy limit between 4.1 and $4.6 \mathrm{MeV}$, which is dependent on the etching conditions and readout criteria. This detector will not detect the plate-out. However, CR-39 detector has a much higher energy threshold for alpha particles and it will register the alpha particles emitted on its surface.

The environmental conditions during the calibration of a detector in the diffusion chamber can be different from those during the actual measurements. Therefore, the deposition behavior of progeny is also different, which can introduce an uncertainty in the measurements. In addition to this uncertainty, there is also the statistical uncertainty of measurements caused by the statistical nature of alphaparticle emission and detection.

As a conclusion, we may emphasize that the irradiation conditions in the chamber are better defined than those for the open detector. In the former case, there are no problems related to the humidity and dust. Problems related to the unknown deposition fraction of ${ }^{218}$ Po can be overcome with proper designs of the chamber. The only disadvantage of the closed detector is that it only carries information about radon, while the open detector carries information about radon and its progeny in air.

\subsubsection{Deposition fraction of ${ }^{218}$ Po inside the diffusion chamber}

As was described earlier, the irradiation geometry and thus the detector response in the diffusion chamber depend on the behavior of ${ }^{218}$ Po inside the chamber. Its deposition changes the geometrical form of the alpha source. Until now, there have been only a few investigations on the partitioning of ${ }^{218}$ Po between the air volume and chamber wall. Exceptions are several theoretical studies but with contradictory conclusions. There are no direct experimental determinations of ${ }^{218}$ Po fraction deposited on the chamber wall.

Theoretical study on ${ }^{218}$ Po deposition was published by McLaughlin and Fitzgerald [128]. They applied the Jacobi room model [129] on the diffusion chamber and came to the conclusion that ${ }^{218} \mathrm{Po}$ was almost completely deposited before decay.

Pressyanov et al. [130] used a different approach to determine the deposition fraction of ${ }^{218}$ Po in the diffusion chamber. The set of diffusion equations that described the behavior of radon and its progeny inside the chamber was:

$$
D \Delta n_{i}-\lambda_{i} n_{i}+\lambda_{i-1} n_{i-1}=0
$$



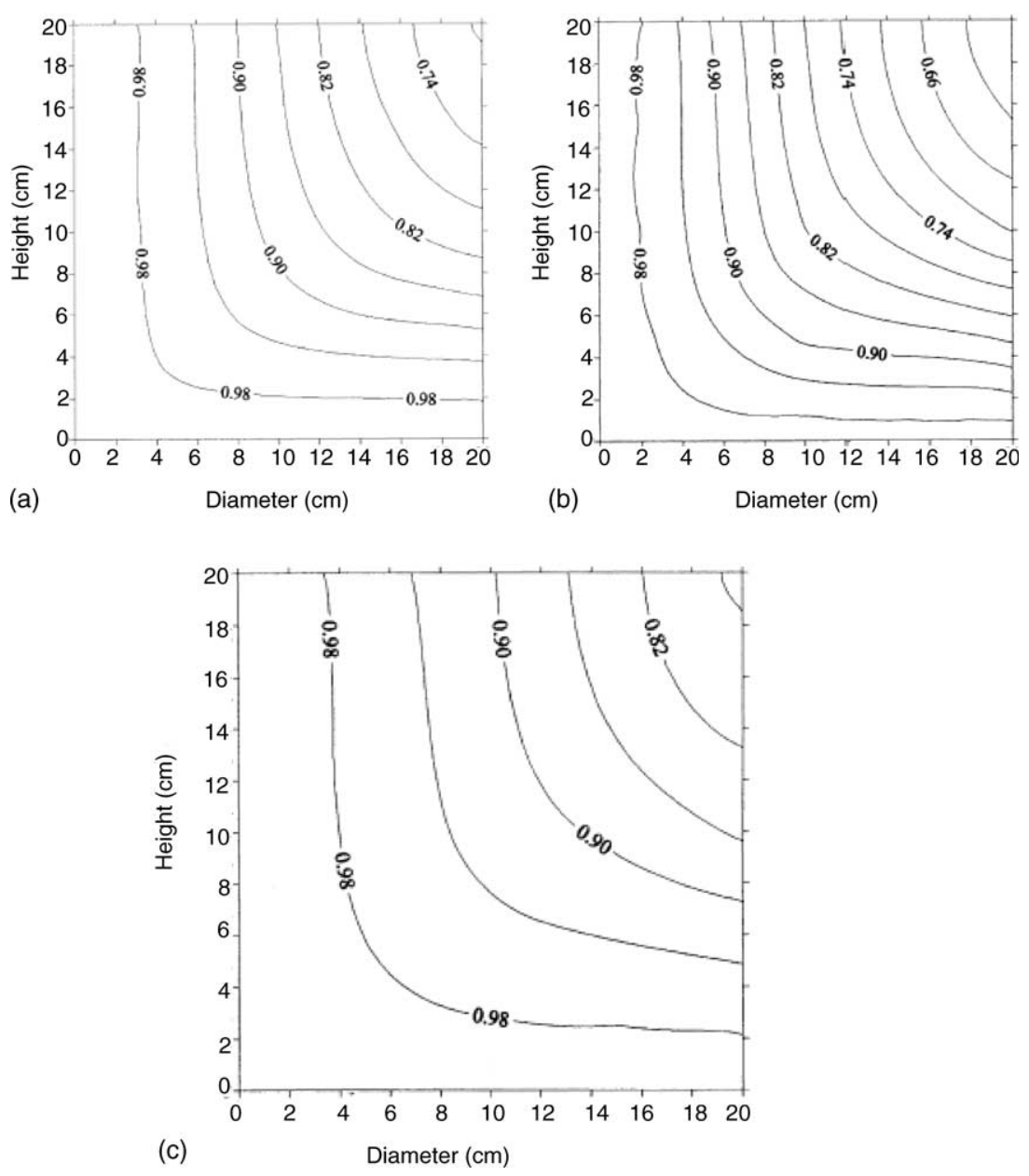

Fig. 38. Deposition fraction of ${ }^{218} \mathrm{Po}$ atoms as a function of the diameter and height of the cylindrical chamber. The lines of equal deposition fraction are drawn for a diffusion coefficient $D$ of: (a) $0.054 \mathrm{~cm}^{2} / \mathrm{s}$; (b) $0.03 \mathrm{~cm}^{2} / \mathrm{s}$; (c) $0.085 \mathrm{~cm}^{2} / \mathrm{s}$ [130].

where $D$ was the diffusion coefficient, $n_{i}$ the atom concentration $(i=0$ for radon and $i=1,2,3$ for the $i$ th radon progeny) and $\lambda_{i}$ were the corresponding decay constants. This set of equations was solved by numerical method for a variety of diameters and heights of cylindrical chambers. Three different diffusion constants of ${ }^{218}$ Po were considered, namely, (a) $0.054 \mathrm{~cm}^{2} / \mathrm{s}$, (b) $0.03 \mathrm{~cm}^{2} / \mathrm{s}$, and (c) $0.085 \mathrm{~cm}^{2} / \mathrm{s}$. The results are given graphically in Fig. 38 as the "equal deposition curves".

Finally, the theoretical results for the calibration factor were compared with the experimental data for metallic and plastic diffusion chambers (dimensions: diameter $D=8 \mathrm{~cm}$ and height $H=7.5 \mathrm{~cm}$ ). The chambers were equipped with LR 115 detectors. The conclusion was that the deposition fraction of ${ }^{218} \mathrm{Po}$ in metallic and plastic chambers were 0.93 and 0.84 , respectively. The experimental value for the detector sensitivity in metallic diffusion chambers was in good agreement with the theoretical results obtained from the diffusion theory with $D=0.054 \mathrm{~cm}^{2} / \mathrm{s}$. The agreement was not good for plastic chambers, which was presumably due to the influence of the static electricity on the freshly formed ${ }^{218}$ Po which was positively charged after formation. The uncertainty introduced by the unknown deposition fraction of ${ }^{218}$ Po was estimated to be below $15 \%$. 
Table 7

Materials and dimensions for the inner surfaces of the diffusion chambers

\begin{tabular}{lllllll}
\hline & \multicolumn{2}{l}{ Chamber material } & & \\
\cline { 2 - 7 } & A (plastic) & B (plastic) & C (tin foil) & D (plastic) & E (acrylic) & $\mathrm{F}(\mathrm{Al})$ \\
\hline$R_{1}(\mathrm{~cm})$ & 2.9 & 3.7 & 3.65 & 2.35 & 2.5 & 2.5 \\
$R_{2}(\mathrm{~cm})$ & 3.1 & 3.9 & 3.65 & 3.3 & 3 & 3 \\
$H(\mathrm{~cm})$ & 8.3 & 10.2 & 7.6 & 4.8 & 5 & 5 \\
$S / V$ ratio $\left(\mathrm{cm}^{-1}\right)$ & 0.9 & 0.7 & 1.2 & 1.1 & 1.1 & 1.1 \\
\hline
\end{tabular}

$R_{1}$ : bottom radius; $R_{2}$ : top radius; $H$ : height. Also shown are the ratios ( $S / V$ ratios) between the total internal surface area and the volumes of the diffusion chambers.

Nikezic et al. [131] pointed out that distribution of track density on the detector placed inside a diffusion chamber was a function of the deposition fraction of ${ }^{218} \mathrm{Po}$. By comparing the experimentally obtained track density distribution and the calculated distribution, they determined the deposition fraction as about 0.6 for a plastic conical diffusion chamber (radii of bases $R_{1}=2.5 \mathrm{~cm}$ and $R_{2}=$ $3.5 \mathrm{~cm}$, and height $H=8.5 \mathrm{~cm}$ ). This concept was also used recently by Koo et al. [132] in the experimental study of track density distribution on the LR 115 detector and the deposition fraction of ${ }^{218} \mathrm{Po}$ in the diffusion chamber. More recently, this approach was further employed to determine the deposition fraction of ${ }^{218} \mathrm{Po}$ in different diffusion chambers [133]. The detector was again LR 115 in order to avoid the influence of plate-out. The experiments were intended to investigate the influence of the chamber size and material on the behavior of ${ }^{218}$ Po deposition. Six diffusion chambers $(A-F)$ were used in the experiments with the parameters given in Table 7.

Experimental track density distributions were obtained after irradiating the detectors and etching in $10 \% \mathrm{NaOH}$ solution at $60{ }^{\circ} \mathrm{C}$ for $120 \mathrm{~min}$, and reading the tracks through a transparent template with concentric circles. The calculated radial track density distributions were obtained using Monte Carlo simulations through computer programs developed earlier by Nikezic and Baixeras [134] and Nikezic and $\mathrm{Yu}$ [135]. In the simulations, the deposition fraction was varied between 0 and 1 with steps of 0.2 in order to fit the experimental data. The results are given in Fig. 39.

From Fig. 39, the following are observed. Interestingly, the best $f$ values for the non-deposited fraction were found to be 0.4 for all six cases. Therefore, the deposition fraction does not depend on the shape and dimensions of the diffusion chambers, nor on the materials of the internal surface of the diffusion chambers.

This finding was in contrast to that of Pressyanov et al. [130] who found dependence on the chamber material. This was also in disagreement with the results of McLaughlin and Fitzgerald [128] which showed almost total deposition of ${ }^{218} \mathrm{Po}$. The deposition fractions determined by different authors were very different. The large discrepancies among different approaches in the determination of the deposition fraction of ${ }^{218}$ Po underlined the necessity for additional experimental and theoretical work on this problem. In addition, there is a problem that has not been tackled yet, neither experimentally nor theoretically; that is the question of uniformity or non-uniformity of deposition of radon progeny inside the chamber.

\subsection{Theoretical models for determination of the calibration factor}

As described earlier, one of the main steps for radon measurements with track detectors is their calibration, i.e., the determination of calibration coefficient in Eq. (4.1). To perform the calibration, one needs an atmosphere with a known radon concentration where the track detectors are exposed for a known period of time. 
There are several review papers on the experimental calibration of solid-state nuclear track detectors for radon measurements, e.g., [136,137], etc. Along with the experimental calibration, different theoretical models for calculating the calibration coefficient were developed by some authors. Until now, there are no critical examinations or comparisons among different theoretical models.
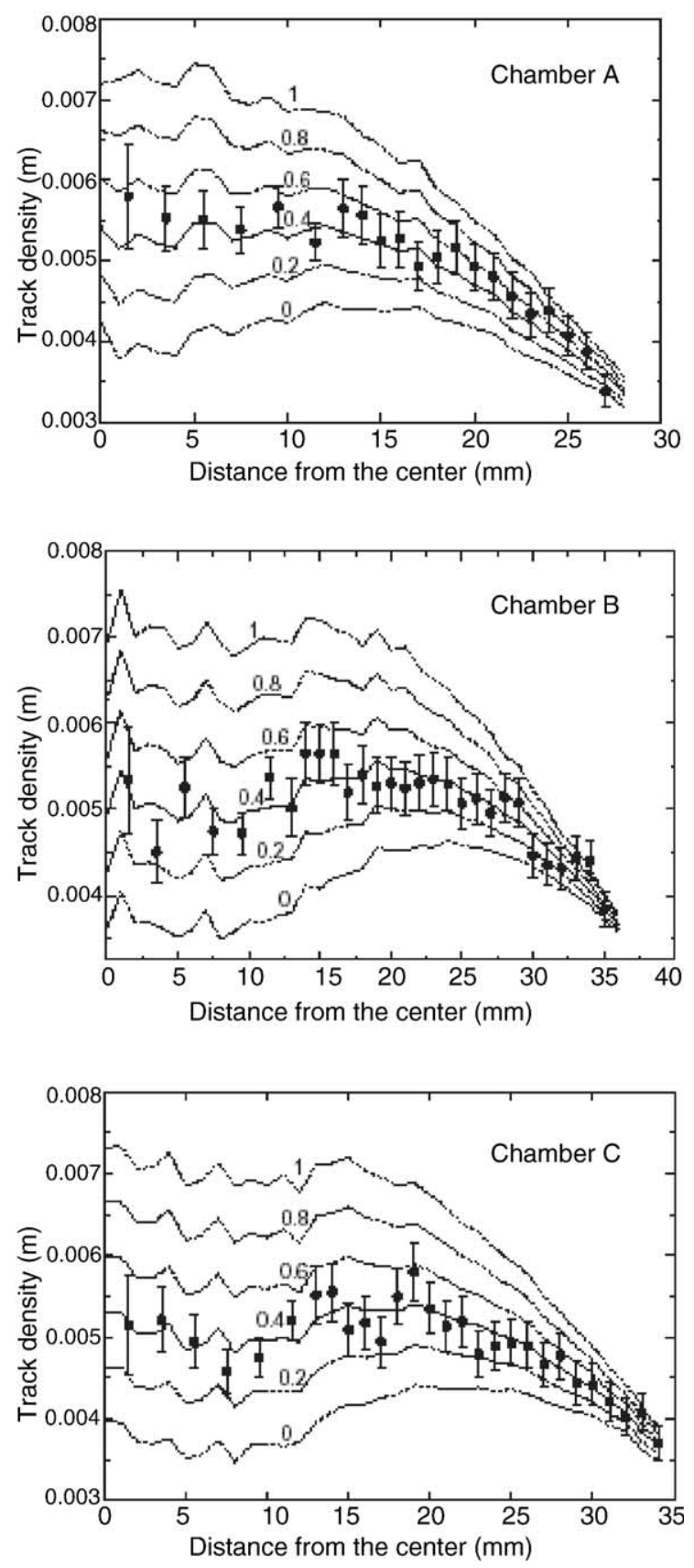

Fig. 39. Experimental data (error bars represent \pm 1S.D.) together with a set of Monte Carlo curves (with different $f$ values shown in the figures) for radial distribution of track densities for chambers A-F [132,133]. The curve for $f=0.4$ is the best fit (in bold) to the experimental data. The uppermost curves (denoted by 1) are obtained under the assumption that no deposition ${ }^{218}$ Po occurs in the chambers (i.e., deposition fraction $=0$ ). The lowest curves (denoted by 0 ) are obtained for total deposition of ${ }^{218}$ Po (i.e., deposited fraction $=1$ ). 

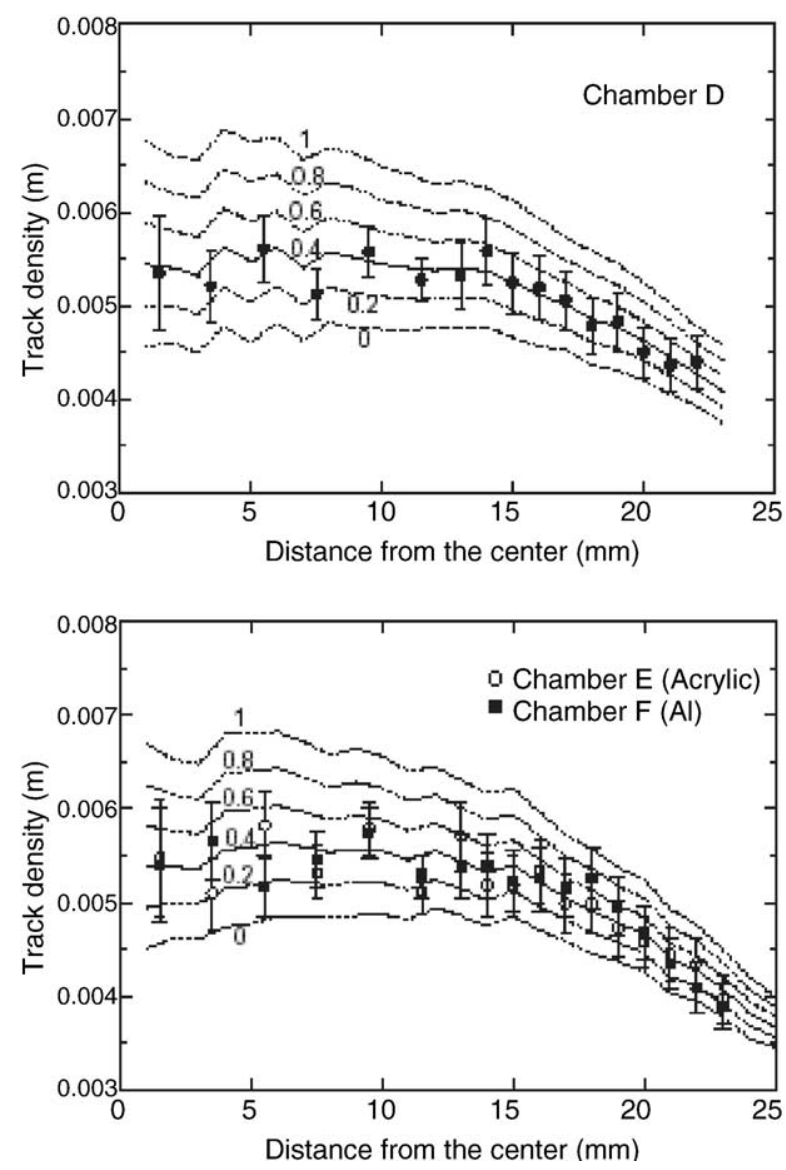

Fig. 39. (Continued).

The theoretical models fall into two categories, viz. analytical calculations and Monte Carlo simulations of alpha-particle detection with track detectors. The theoretical models enable investigation of some aspects that are not possible with the experimental approach. For example, it is not easy to distinguish among tracks from different alpha emitters in the radon chain experimentally. Consequently, one cannot determine the influence of different progeny on the total detector response. However, this is possible with the use of some theoretical models.

\subsubsection{Analytical approaches}

Analytical calculations of the detection probability were performed for open as well as for closed detectors. In the case of open detectors, an upper limit on the detector sensitivity may be calculated based on the Cherry's formula:

$$
J=\frac{R A}{4}
$$

where $J$ is the number of alpha particles impacted on the detector, $R$ is the $\alpha$ particle range in air (in $\mathrm{cm}$ ) and $A$ is the concentration of $\alpha$ emitting radionuclides in air $\left(\mathrm{Bq} / \mathrm{cm}^{3}\right)$. There are three $\alpha$ emitters in the ${ }^{222} \mathrm{Rn}$ chain with corresponding $\alpha$ energies of $5.49 \mathrm{MeV}\left({ }^{222} \mathrm{Rn}\right), 6 \mathrm{MeV}\left({ }^{218} \mathrm{Po}\right)$ and $7.69 \mathrm{MeV}$ $\left({ }^{214} \mathrm{Po}\right)$. Their ranges in air are $4.09,4.67$ and $6.78 \mathrm{~cm}$, respectively. According to the previous data and assuming that the concentrations of radon and its progeny in air are all $1 \mathrm{~Bq} / \mathrm{m}^{3}$, the maximum track 
numbers are given by:

$$
\begin{aligned}
\rho_{\mathrm{Rn}-222} & =0.0883\left(\text { tracks } / \mathrm{cm}^{2}\right) /\left(\mathrm{Bq} \text { day } / \mathrm{m}^{3}\right) \\
\rho_{\mathrm{Po}-218} & =0.1\left(\text { tracks } / \mathrm{cm}^{2}\right) /\left(\mathrm{Bq} \text { day } / \mathrm{m}^{3}\right) \\
\rho_{\mathrm{Po}-214} & =0.146\left(\text { tracks } / \mathrm{cm}^{2}\right) /\left(\mathrm{Bq} \text { day } / \mathrm{m}^{3}\right)
\end{aligned}
$$

The total track density is the sum of the partial track densities (assuming equilibrium between radon and its progeny) as:

$$
\rho_{\text {total }}=0.334\left(\text { tracks } / \mathrm{cm}^{2}\right) /\left(\text { Bq day } / \mathrm{m}^{3}\right)
$$

This result may be taken as the upper limit on the sensitivity of an open detector. The plate-out of radon progeny has not been taken into account in this calculation. The real number of tracks after etching will be smaller than the prediction using Eq. (4.5) because etching will not transform all latent tracks into visible tracks. In the case of the closed detector, the existence of the chamber wall changes the irradiation geometry and disturbs the earlier considerations.

Fleischer and Mogro-Compero [138] described a method based on the equation:

$$
\Delta=\frac{1}{4} \iint A(r, \theta) \sin 2 \theta \mathrm{d} r \mathrm{~d} \theta
$$

where $\Delta$ is the track density per unit exposure time and $A$ is radioactivity of a radionuclide $\left({ }^{222} \mathrm{Rn}\right.$, ${ }^{218} \mathrm{Po}$ and ${ }^{214} \mathrm{Po}$ in the case of radon measurements). The integration has to be performed along the effective volume from which the particles can be detected by a detector. Since the activity in air $A=$ constant, the previous integral becomes:

$$
\Delta=\frac{A}{4} \iint \sin 2 \theta \mathrm{d} r \mathrm{~d} \theta=A C
$$

where $C$ is a constant. This equation is similar to the Cherry's formula. The difference is the introduction of the notion of the effective volume, which is defined as the volume in front of the detector from which $\alpha$ particles emitted have a detection probability larger than 0 (or have a non-zero detection probability). Particles emitted beyond the effective volume cannot be detected, i.e., their detection probability is equal to zero. The shape and size of the effective volume depend on the $\alpha$ particle energy, the etching condition, readout procedures and the size and shape of the detector. Since three groups of $\alpha$ particles are emitted in ${ }^{222} \mathrm{Rn}$ chain, three different effective volumes have to be determined (for the same etching conditions).

Other published analytical formulas are variants of Eq. (4.6). For example, Bagnoli et al. [139] used the following formulas for the sensitivity of LR 115 detectors in the chamber designed by Tommasino [140] (i.e., two LR 115 detectors facing each other and covered with Mylar foils, and placed in small plastic chambers):

$$
\begin{aligned}
\varsigma_{\mathrm{V}}^{i} & =\frac{C}{S^{\prime}} \int_{S^{\prime}} \int_{V} \frac{\cos (\theta) \eta\left(E\left(E_{0}^{i}, r, \theta, s\right), \theta\right)}{4 \pi r^{2}} \mathrm{~d} S^{\prime} \mathrm{d} V \\
\varsigma_{\mathrm{S}}^{i} & =\frac{C}{S^{\prime}} \int_{S^{\prime}} \int_{S} \frac{\cos (\theta) \eta\left(E\left(E_{0}^{i}, r, \theta, s\right), \theta\right)}{4 \pi r^{2}} \cos (\psi) \mathrm{d} S^{\prime} \mathrm{d} V
\end{aligned}
$$

where $\zeta_{\mathrm{V}}^{i}$ and $\zeta_{\mathrm{S}}^{i}$ are detector sensitivities to the alpha emitters in the chamber volume and on inner surfaces, respectively, $C$ is a constant, $S^{\prime}$ is the counting detector area, $\theta$ is the incidence angle, $E_{0}^{i}$ the 
energy of emission of the $i$ th radionuclide, $E\left(E_{0}^{i}, r, \theta, s\right)$ the energy of alpha particles at the detector surface (after traveling distances $r$ in air and $s$ in the absorber), $\eta(E, \theta)$ the LR 115 detector efficiency and $\psi$ is the angle between the chamber surface and the alpha-particle trajectory. The computation of $E\left(E_{0}^{i}, r, \theta, s\right)$ is based on the ranges of alpha particles in air and in the Mylar foil, which are calculated by means of the Bethe-Bloch formula for stopping power. The computation has to be performed numerically. The detector sensitivity $\eta$ was obtained by interpolation between experimentally data obtained by Marocco and Bochicchio [141]. The results on the detector sensitivity were presented as a function of the thickness of the Mylar foil for two extreme cases: (a) uniform radon progeny distribution in the chamber volume and (b) uniform radon progeny deposition on the surface. The agreement between theoretical and experimental data was somewhat better for the assumption (b), but there was still some discrepancy which was attributed to the non-uniform deposition of the radon progeny.

\subsubsection{Monte Carlo calculations of the detector sensitivity}

Along with the analytical approach, some authors applied Monte Carlo simulations to calculate the detector sensitivity. The procedures of simulation were described in different papers. Here, we will focus on some differences among these works. Some authors used the effective volume, which had to be determined prior to the main simulations. The initial points of alpha-particle emission were then sampled only in that volume. Other authors sampled initial points in a hemisphere around the detector with the radius equal to the range of alpha particles.

The decision on whether a particle is detected or not (i.e., whether it will produce a visible track after etching) is also different in various works. In some papers, the main criterion is the critical angle. A particle will be detected if it strikes the detector above the critical angle, and vice versa. However, the critical angle is a function of the particle energy and the etching condition. Furthermore, this function is rather complicated and no single value for the critical angle can be assumed. An example showing the complex nature of the function for the CR-39 detector has been given by Dorschel et al. [142] and is reproduced here in Fig. 40.

There are some irregularities in the curves shown in Fig. 40 for the initial alpha energy larger than 5.5 MeV. Also, the critical angle decreases significantly for energies below $1.5 \mathrm{MeV}$.

Another possible approach in making decisions for detection is to calculate the track parameters (major and minor axes of track openings and depths of tracks) for each particle striking the detector.

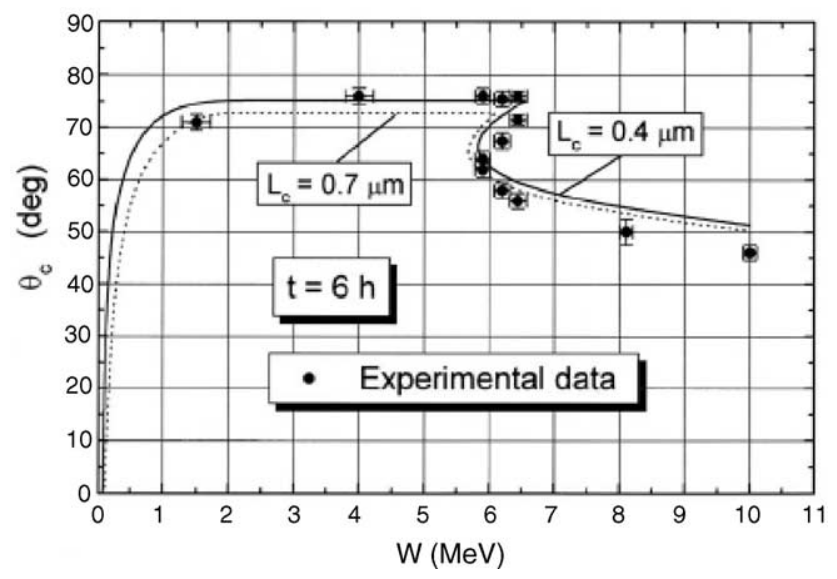

Fig. 40. The critical angle $\theta_{\mathrm{c}}$ computed as a function of the initial alpha energy $W$ for an etching time of $t=6 \mathrm{~h}$ and spatial resolutions of $L_{\mathrm{c}}=0.4$ and $0.7 \mu \mathrm{m}$, respectively, in comparison with the experimental results (circles) [142]. 
These calculations are based on the etching conditions (expressed in the thickness of the removed layer of the detector during etching), on the known $V_{\mathrm{t}} / V_{\mathrm{b}}$ function, etc. Based on the calculated track parameters, one should then decide whether the track is visible or not.

\subsubsection{Approaches based on the effective volume}

In the ${ }^{222} \mathrm{Rn}$ decay chain, three groups of alpha particles are emitted. This means that for any kind of detector and any particular removed layer and readout procedures, three different effective volumes must be defined, one for each alpha-particle energy. Such work has been performed by Damkjaer [143] for a point-like detector for all three alpha-particle energies. However, Damkjaer [143] gave the isoefficiency curves for $\varepsilon=0.5$ (efficiency). The procedures for determination of an effective volume are described further.

First, the effective volume for a "point-like" detector has to be found by examining the space of hemisphere with radius $R$ above the detector ( $R$ is the alpha-particle range in air). By varying the incidence angle and distance with small steps, the assignments, or otherwise, of points above the detector to the effective volume are performed systematically. The limits of the effective volume $\varepsilon>0$ (inside the volume) and $\varepsilon=0$ (outside the volume) for an LR 115 detector are given in Fig. 41 [144]. The effective volume for the point detector is obtained by rotating the graphs shown in Fig. 41 around the $z$-axis, and has an interesting shape.
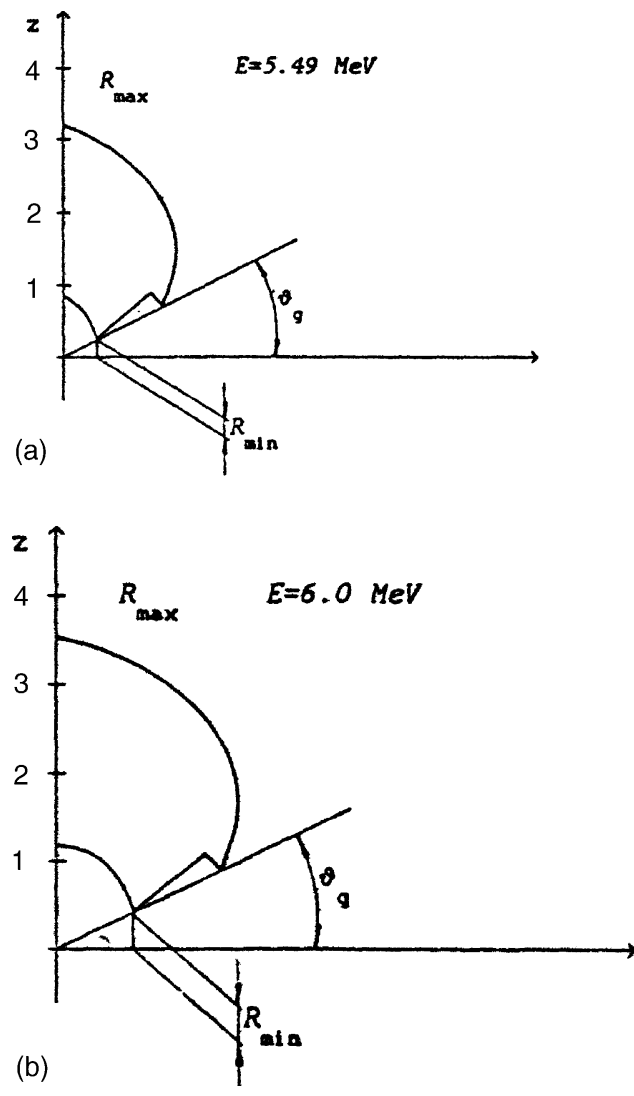

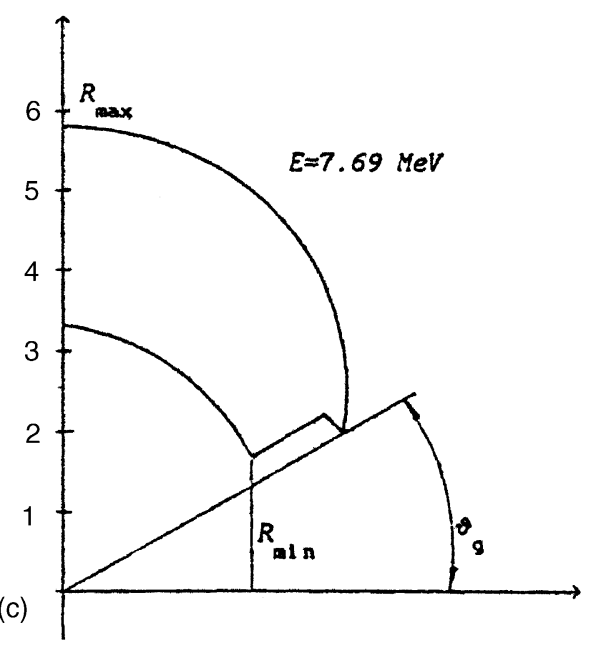

(d)

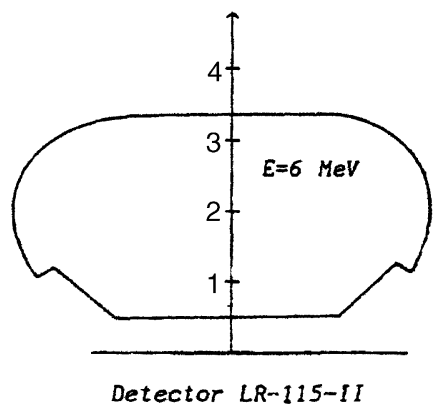

Fig. 41. Effective volumes of point-like LR 115 detectors for a removed layer of $4.7 \mu \mathrm{m}$ for alpha particles with energies of: (a) $5.49 \mathrm{MeV}$; (b) $6.0 \mathrm{MeV}$; (c) $7.69 \mathrm{MeV}$. (d) The cross-section of the effective volume for a rectangular LR 115 detector for alpha particles of $6 \mathrm{MeV}$ (adopted from [144]). 


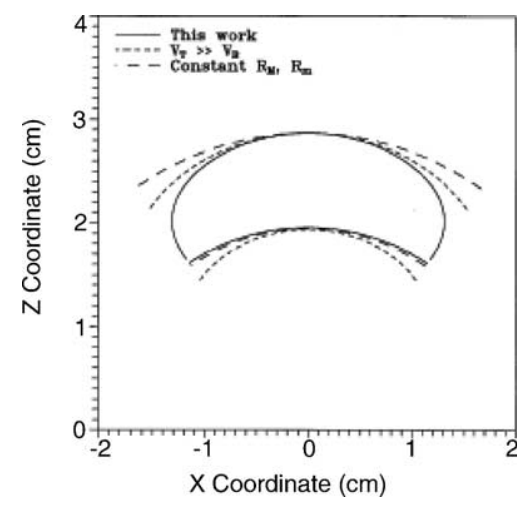

Fig. 42. The sensitive volume for ${ }^{222} \mathrm{Rn}$ detection in air using the LR 115 detector etched to have a removed layer of $5 \mu \mathrm{m}$. The detector is considered a disc parallel to the $x y$-plane with the center of the exposed side at the origin of the coordinate system [145].

For the real detector, with dimensions of several centimeters, the effective volume is the sum of effective volumes of all detector points. Extrapolating the effective volume from the point to the real detector is not a trivial problem. The effective volume just above the detector has a prismatic shape with the base equal to the detector. The lower base of the prism is at the distance $R_{\min }$ and the upper base is at the distance $R_{\max }$ from the detector (as shown in Fig. 41). The cross-section of the effective volume for a rectangular LR 115 detector for alpha particles of $6 \mathrm{MeV}$ is also shown in Fig. 41(d).

It is noted that alpha particles need to lose some energy in air before they satisfy the upper energy threshold of the LR 115 detector. When the CR-39 detector is considered, there is no such requirement; the upper energy threshold for alpha detection is much larger than the alpha energy in the ${ }^{222} \mathrm{Rn}$ decay chain. There are also other works which deal with the effective volume for the LR 115 detector. For example, Sima [145] presented a somewhat different shape of the effective volume for the LR 115 detector as shown in Fig. 42. The effective volume presented in Fig. 42 is significantly smaller that that in Fig. 41(a). The difference is probably due to the different energy windows used by the authors.

Andriamanatena et al. [146] presented the effective volumes for the LR 115 detector for two different etching conditions. Their results are presented in Fig. 43. The first etching conditions were $10 \% \mathrm{NaOH}$ at $60{ }^{\circ} \mathrm{C}$, for which the bulk etch rate $V_{\mathrm{b}}=3.4 \pm 0.1 \mu \mathrm{m} / \mathrm{h}$ was assumed. The authors also assumed the $V_{\mathrm{t}}$ function as:

$$
V_{\mathrm{t}}=V_{\mathrm{b}}\left(1+\mathrm{e}^{-0.16 R+2.68}\right)
$$

The second etching conditions were $6 \mathrm{~N} \mathrm{NaOH}$ at $60{ }^{\circ} \mathrm{C}$ for $2.5 \mathrm{~h}$ with:

$$
V_{\mathrm{t}}=V_{\mathrm{b}}\left(1+\mathrm{e}^{-0.00688 R+2.61}\right)
$$

(a)

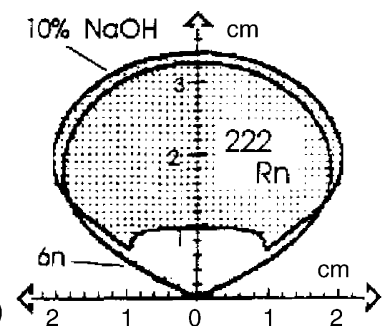

(b)

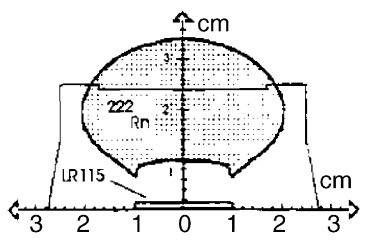

Fig. 43. (a) Effective volumes for the LR 115 detector for alpha particles from ${ }^{222} \mathrm{Rn}$ for two different etching conditions. (b) The effective volume with the cross-section of a Lund cup diffusion chamber (adopted from [146]). 

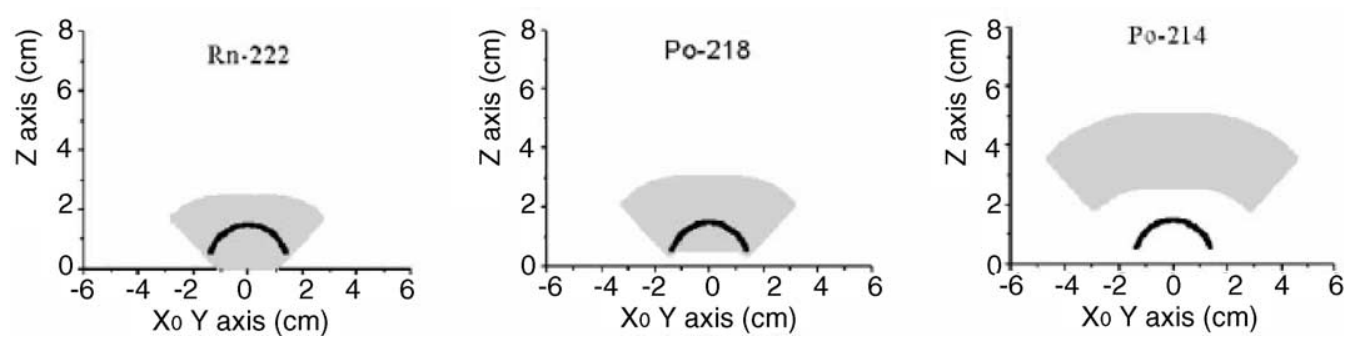

Fig. 44. Effective volumes for the ${ }^{222} \mathrm{Rn}$ decay chain [147]. The energy window is between 3 and $5.5 \mathrm{MeV}$. The black lines represent the wall of the hemispherical diffusion chamber.

and $V_{\mathrm{b}}=2.54 \pm 0.11 \mu \mathrm{m} / \mathrm{h}$. Here we can see that the effective volume, i.e., the form and size of the radioactive source, depends on the etching condition. In the case of ${ }^{222} \mathrm{Rn}$, only part of the source contributes to the total track density.

Effective volumes have also been determined for other types of detectors (other than LR 115). For example, Amgarou et al. [147] presented the effective volume for the Makrofol detector (with an energy window between 3.0 and $5.5 \mathrm{MeV}$ ) for the radon and thoron decay chains (Fig. 44).

A horizontal part of the effective volume can be seen just above the detector (similarly to that obtained in [144]). In Fig. 44, intersections between the chamber walls and some effective volumes are present, for which cases the alpha particles emitted from the wall can be detected. Calculations of the track densities and detector sensitivities have to be performed separately for radon progeny in air and radon progeny on the walls. The following formulas are used to calculate the track density $\left(\mathrm{in} \mathrm{m}^{-2}\right.$ ) on the detector in the diffusion chamber:

$$
\rho_{\mathrm{a}}=\frac{\varepsilon_{\mathrm{a}} V A_{\mathrm{V}} t}{\pi r_{\mathrm{det}}^{2}}
$$

for alpha particles emitted in air, and

$$
\rho_{\mathrm{W}}=\frac{\varepsilon_{\mathrm{W}} S A_{\mathrm{S}} t}{\pi r_{\mathrm{det}}^{2}}
$$

for alpha particles emitted from the surfaces of the diffusion chamber, where $\varepsilon_{\mathrm{a}}$ and $\varepsilon_{\mathrm{W}}$ are the detector sensitivities for progeny emitted in air and from wall, respectively, $V$ is the effective volume in $\mathrm{m}^{3}, A_{\mathrm{V}}$ is the volume concentration of alpha emitters, $S$ is the effective surface (in $\mathrm{m}^{2}$ ), $A_{\mathrm{S}}$ the surface activity of a given radionuclide, $t$ is the exposure time, and $r_{\mathrm{det}}$ is the radius of the detector. The ratio of the track density (in $\mathrm{m}^{-2}$ ) and the total exposure (in $\mathrm{Bq} \mathrm{s} / \mathrm{m}^{3}$, assumed to be $1 \mathrm{~Bq} \mathrm{~s} / \mathrm{m}^{3}$ ) gives the sensitivities $\rho_{i}$ in $(\mathrm{m})$ for the alpha particles emitted in air and from the walls. The total sensitivity $\rho_{\text {tot }}$ of the detector is the sum of the partial sensitivities multiplied by factors $f_{i}$ which represent the partitioning of radon progeny between air and the wall and given by the following equation:

$$
\rho_{\text {tot }}=\rho_{0}+f_{1} \rho_{1 \mathrm{a}}+f_{4} \rho_{4 \mathrm{a}}+\left(1-f_{1}\right) \rho_{1 \mathrm{~W}}+\left(1-f_{4}\right) \rho_{4 \mathrm{~W}}+p_{1} N_{1}+p_{4} N_{4}
$$

where $\rho_{i \mathrm{a}}$ (in m) is the corresponding sensitivity of ${ }^{222} \mathrm{Rn}(i=0),{ }^{218} \mathrm{Po}(i=1)$ and ${ }^{214} \mathrm{Po}(i=4)$ from air, and those with subscript ' $w$ ' the counterparts from wall, $p_{i}$ is the probability for plate-out detection, and $N$ the number of progeny atoms decayed on the detector per unit time and unit radon activity in air. In the case of the LR 115 detector, $p_{i}=0$, i.e., there is no detection of plate-out. 

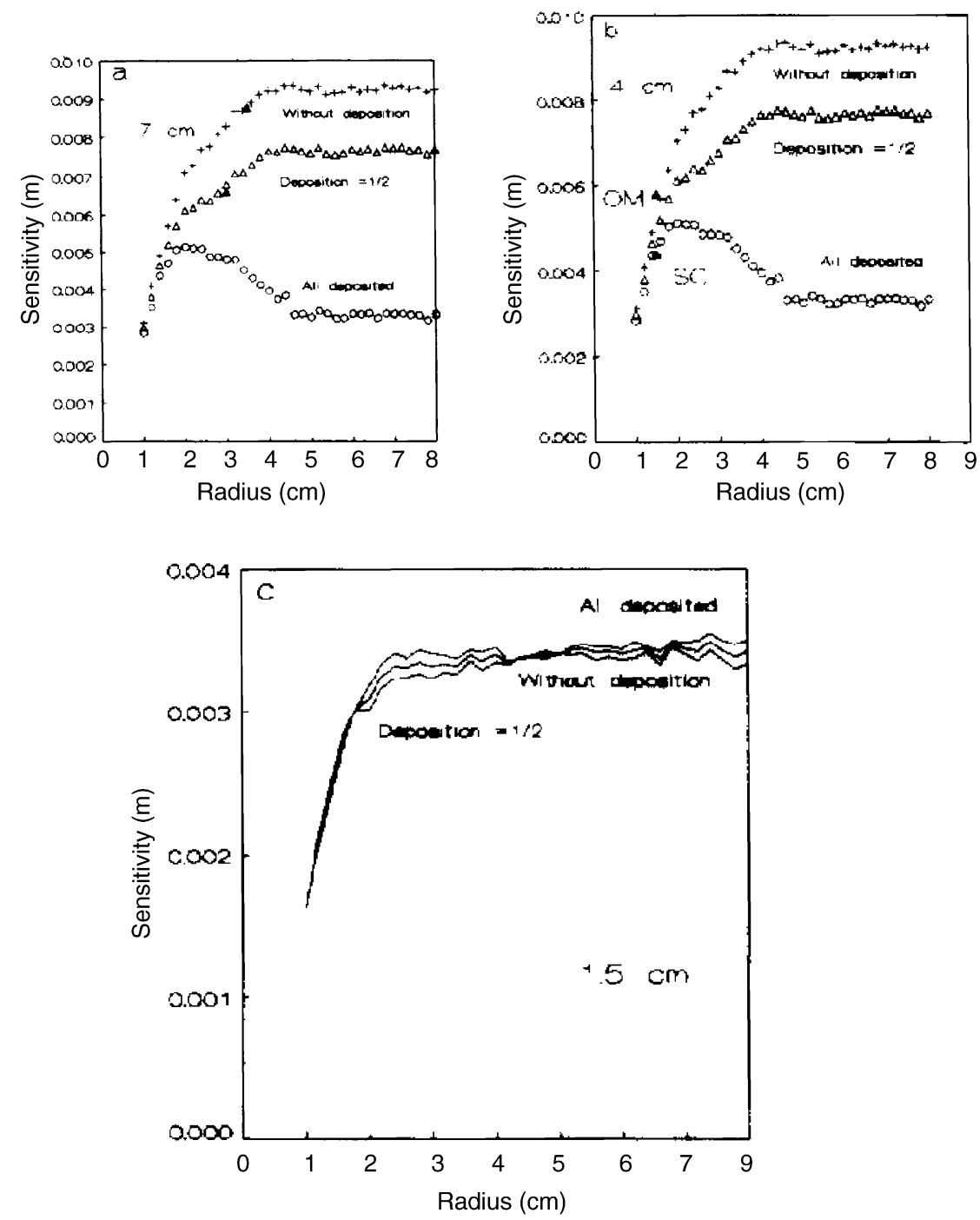

Fig. 45. The total LR 115 detector sensitivities for different fractions of deposited radon progeny in different tubes, as functions of the tube radius [134]. Experimental values are given as solid triangles in (a) from [148] and in (b) from [149].

\subsubsection{Some results from theoretical calculations}

Calculations of the detector sensitivity have enabled investigation of the dependence of the detector response on the diffusion chamber size and shape, and also enabled studies of the uncertainty introduced by the unknown deposition of ${ }^{218} \mathrm{Po}$, etc. As an example, the total LR 115 detector sensitivities in cylindrical diffusion chambers are given in Fig. 45. The sensitivity is given as a function of the chamber radius for three different chamber heights, i.e., $h=7 \mathrm{~cm}$ (Fig. 45(a)), $4 \mathrm{~cm}$ (Fig. 45(b)) and $1.5 \mathrm{~cm}$ (Fig. 45(c)) [134]. There are three curves in each of these figures obtained for different assumptions of the deposited amount of ${ }^{218} \mathrm{Po}$.

One can see that the sensitivity decreases when the chamber is smaller. However, the uncertainty introduced by the unknown deposition fraction of ${ }^{218}$ Po also decreases. From Fig. 45(c), i.e., the results for $h=1.5 \mathrm{~cm}$, it can be seen that measurements independent of the ${ }^{218}$ Po behavior are enabled. Some comparisons with experimental data are also given in Fig. 45(a) and (b). 


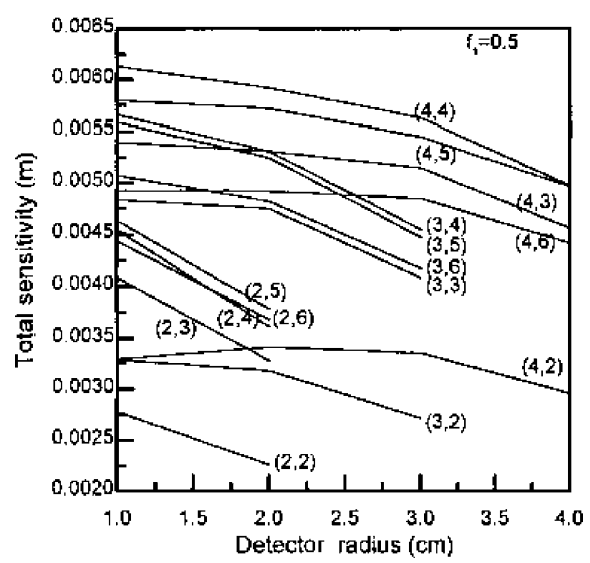

Fig. 46. The variation of the total sensitivity of the LR 115 detector in diffusion chambers with different chamber dimensions as a function of the detector radius [135]. The dimensions of the chambers are denoted by a pair of numbers $(R, H)$ where $R$ (in $\mathrm{cm}$ ) is the chamber radius and $H$ (in $\mathrm{cm}$ ) is the height of the chamber. The results have been calculated under the assumption $f=0.5$ (i.e., half of ${ }^{218} \mathrm{Po}$ is deposited before decay).

The detector sensitivity also depends on the size of the detector inside the diffusion chamber. This has been theoretically investigated by Nikezic and Yu [135] and the results are given in Fig. 46. It can be seen that the detector sensitivity decreases with the detector's radius, since the track density decreases as the distance from the center increases.

\subsection{Radon progeny measurements with track detectors}

It has been well established that the absorbed radon dose in the human lung is not delivered by radon itself, but instead mostly caused by its short-lived progeny. The absorbed dose is commonly determined by first measuring the radon concentration and then applying an assumed equilibrium factor between radon and its progeny (usually between 0.4 and 0.5 ). This forms the basis for determining the exposure to radon progeny expressed in the traditional unit working level month (WLM). The exposure can then be multiplied with the dose conversion coefficient, which is nominally recommended to be $5 \mathrm{mSv} / \mathrm{WLM}$, to give the effective dose [150].

A drawback for these procedures is the requirement to assume an equilibrium factor. Until now, there is no widely accepted methods for long-term passive measurements of radon progeny concentrations, despite the fact that short-term active measurements are relatively easy by air filtering and subsequent activity measurements of the filter. Active methods based on air filtering only give radon progeny concentrations in short time intervals. Since the concentrations of radon and its progeny vary significantly with time, the knowledge of concentrations in one short time interval might not be very useful. It would be of great interest to use solid-state nuclear track detectors to measure the indoor ${ }^{222} \mathrm{Rn}$ progeny concentrations in the same period during which the ${ }^{222} \mathrm{Rn}$ gas concentration is obtained by using the diffusion chamber. For this reason, exploration of long-term passive integration measurement methodologies regarding short-lived radon progeny has attracted much attention in the field of radon dosimetry.

There were many proposals for utilizing of SSNTDs in radon progeny measurements. A brief review was given by Amgarou et al. [151]. The first method was proposed by Frank and Benton [152]. In this method, two detectors were used, one in a diffusion chamber and a second one in the open mode. The open detector registers the total concentration of $\alpha$-particle emitting nuclei in air, i.e., ${ }^{222} \mathrm{Rn}$ + progeny $\left({ }^{218} \mathrm{Po}+{ }^{214} \mathrm{Po}\right)$, while the closed detector measures only ${ }^{222} \mathrm{Rn}$. The ratio between the 
readings in open and closed detectors is related to the equilibrium factor and from here it should be possible to estimate the equilibrium factor. This method was used by many authors under different detector setups, but did not give realistic results. The presence of ${ }^{220} \mathrm{Rn}$ and its progeny and plate-out effect disturbed the measurements. This method assumed the same sensitivity for the open and closed detector for each of the $\alpha$ emitting nuclei in the ${ }^{222} \mathrm{Rn}$ decay chain. As shown earlier, the sensitivity of the closed detector depends on the chamber shape and dimension and may be very different from the sensitivity of an open detector.

Another method was proposed by Fleischer [153] who used four detectors for progeny measurements. Three detectors were covered with polyethylene absorber foils with different thickness. The bare detector and the first one with $20 \mu \mathrm{m}$ absorber can detect alpha particles emitted by ${ }^{222} \mathrm{Rn}$ and the progeny ${ }^{218} \mathrm{Po}+{ }^{214} \mathrm{Po}$. Another two with thicker absorbers measure $\alpha$ emissions from ${ }^{218} \mathrm{Po}+{ }^{214} \mathrm{Po}$ and ${ }^{214} \mathrm{Po}$. By this method, the $\alpha$-active progeny $\left({ }^{218} \mathrm{Po}\right.$ and $\left.{ }^{214} \mathrm{Po}\right)$ are measured separately. The concentrations of $\beta$ emitting nuclei ${ }^{214} \mathrm{~Pb}$ cannot be measured with track detectors. However, this method fails because of unavoidable progeny deposition on the detectors and on the absorbers foils.

Other methods are based on the assumption that the equilibrium factor depends only on the ventilation rate. In fact, several processes affect the ratio of progeny concentrations to the radon concentration, such as deposition and ventilation. The model of Jacobi [129] describes the balance of radon and its progeny in a closed space. If all other parameters are kept at their the best estimates, from the ratio of the track density on the open and closed detectors, one could infer the ventilation rate and the equilibrium factor [154]. However, this method fails for two reasons. The equilibrium factor is a weak function on the ventilation rate. Actually, the radon concentration is very dependent on the ventilation rate, but the ratio of progeny concentrations to the radon concentration is not. The dependence of the equilibrium factor on the ventilation rate is shown in Fig. 47.

For some "normal" ventilation rates between 0.5 and $1.5 \mathrm{~h}^{-1}$, the equilibrium factor varies between 0.32 and 0.23 . This change is very small if we take into account other uncertainties, such as statistical errors in the number of the tracks, etc. Another reason why this method fails is that the deposition rates of radon progeny can be very different from the "typical" values. Dorschel described this method as "very inaccurate" [155].

One extension of this method was proposed by Dorschel and Piesch [156]. In this method, special etching conditions have to be applied on the track detector so that the energy window is between 6.05

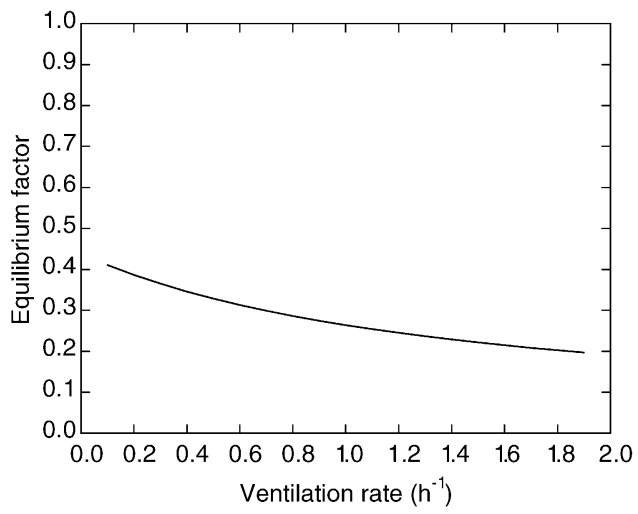

Fig. 47. Dependence of the equilibrium factor on the ventilation rate. The results are obtained under the following conditions: attachment rate of ${ }^{218} \mathrm{Po}$ is $50 \mathrm{~h}^{-1}$; deposition rate of unattached and attached progeny are 20 and $0.2 \mathrm{~h}^{-1}$, respectively; the recoil factor of ${ }^{214} \mathrm{~Pb}$ is 0.83 . 
and 7.5 MeV. In this way, only ${ }^{214} \mathrm{Po}$ in air was measured. From the ratio of concentrations between ${ }^{214} \mathrm{Po}$ and ${ }^{222} \mathrm{Rn}$, one can obtain the equilibrium factor. This method was also based on the assumption that all other parameters were kept at their best estimations except the ventilation rate. The method is better than the previous one but there is a difficulty to realize such a narrow energy window. The calibration is also another problem for this method because it is not easy to obtain air contaminated only with ${ }^{214}$ Po. Here, the calibration factor has to be calculated.

Nikezic and Baixeras [157] proposed the barrier method for which barriers are placed at some distances in front of the detector. Due to the differences in the alpha energies emitted from different progeny and the existence of an upper limit in the energy window for the LR 115 detector, separation of progeny has been shown to be theoretically possible. An alpha particle has to travel some distances in the air before its energy falls below the upper limit in the energy window to be detected. If the barrier is too close, the LR 115 detector will not detect any alpha particle. If the barrier is a little bit farther away, it will detect alpha particles from radon only, and so on. However, experimental verification of this method was not successful [158]. The reason was the radon progeny plate-out on the barrier, for which the theoretical model failed to describe.

Another class of methods is based on spectrometry of alpha particles. From the track parameters, it is possible to determine the energy of alpha particles that produce the tracks. However, the track parameters are dependent on the incident angle in additional to the incident energy. Therefore, the same track parameters (major and minor axes) can be obtained with different incident energies. Other track parameters are needed, such as the average gray level or the track shape. Even the establishment of the incident alpha-particle spectrum from the track parameters is difficult and tedious. The second step would be the interpretation of the data and linking the obtained spectra with the equilibrium factors. The incident alpha-particle spectrum depends on the relative concentrations of radon progeny in air. In Fig. 48, the incident alpha particle spectrum is given, where $f(E)$ refers to the fraction of alpha particles incident on the detector with an energy between $E$ and $E+\mathrm{d} E$ [159].

In Fig. 48, the spectra for two extreme cases are given, namely, for an equilibrium factor $F=1$ and for a low equilibrium factor $F=0.1$. The curves in Fig. 48 show a non-Gaussian peak at $5.49 \mathrm{MeV}$ and cutoff structures above $5.49 \mathrm{MeV}$. The first cutoff from right to left is related to ${ }^{214} \mathrm{Po}$ while the second to ${ }^{218} \mathrm{Po}$. The curves have plateaus between 6 and $7.69 \mathrm{MeV}$ and between 5.49 and $6 \mathrm{MeV}$; the former $\left({ }^{214} \mathrm{Po}\right.$ plateau $)$ is wider because of the wider energy gap, while the latter $\left({ }^{218} \mathrm{Po}\right.$ plateau $)$ is narrower and higher than the ${ }^{214}$ Po plateau. By analyzing the height of these two plateaus, one can infer some

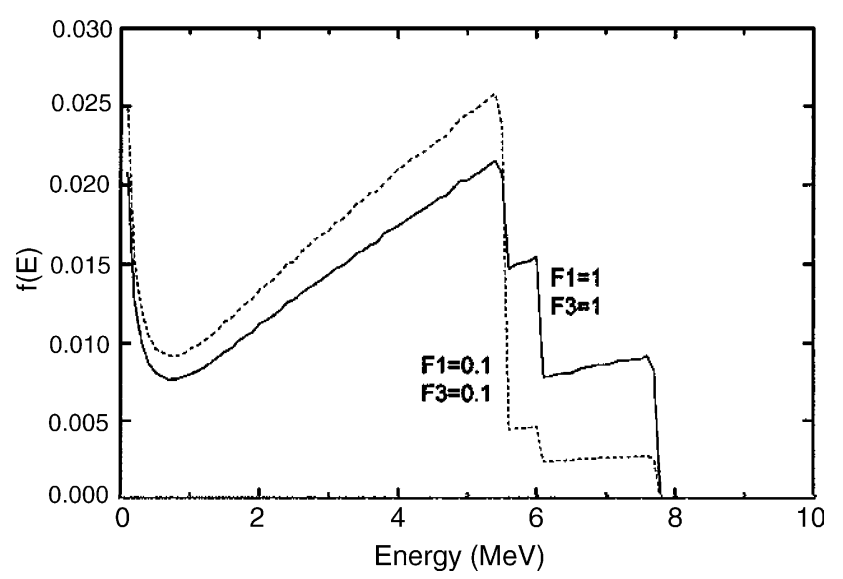

Fig. 48. Alpha particle spectra for different relative ratios of progeny to radon concentrations [159]. 
information about the relative activities of ${ }^{218} \mathrm{Po}$ and ${ }^{214} \mathrm{Po}$. If their activities are equal (as shown in Fig. 48 , where $F_{1}=F_{3}$ ), the ${ }^{218}$ Po plateau is two times higher than the ${ }^{214}$ Po plateau.

The process of plate-out will create two peaks at 6 and $7.69 \mathrm{MeV}$ in the measured spectrum. The heights of these peaks depend on the amount of plate-out as well as the efficiency and energy resolution of the detector and the measuring conditions. The most important part of the spectrum is between 6 and $7.69 \mathrm{MeV}$. Here, only ${ }^{214} \mathrm{Po}$ from air contributes to the detector response. In order to avoid the influence of plate-out (if SSNTDs are considered), the energy window between 6.2 and 7.5 should be considered. Actually the width of the window depends on the energy resolution attainable in measurements, but of course, it should not be wider than the range from 6 to $7.69 \mathrm{MeV}$. If the energy resolution is worse than $1 \mathrm{MeV}$, measurements of radon progeny would not be possible by analyzing the energy spectrum. The feasibility of radon progeny measurement relies on the provision of high-resolution alpha spectroscopy. Even if only ${ }^{214} \mathrm{Po}$ is measured, that is not yet an equilibrium factor. From this short analysis, it is seen that the methods based on alpha spectroscopy are rather difficult and should be used with a rather high uncertainty. One extension of the spectroscopy method is to measure only tracks with the ratio of major to minor axes in some limits. However, this method is very tedious, and finally the number of tracks that satisfies the given condition is rather small. Then, the statistical error is large.

From the earlier discussion, it is seen that up to now there is still no accurate, practical and widely used methods for long-term measurements of the equilibrium factor with nuclear track detectors. All of the above-mentioned methods suffer from some kind of problems.

Recently Amgarou et al. [151] proposed measurements of the equilibrium factor, which is given by:

$$
F_{222 \mathrm{Rn}}=0.105 f_{218} \mathrm{Po}+0.515 f_{214} \mathrm{~Pb}+0.380 f_{214} \mathrm{Bi}, \mathrm{Po}
$$

through the "reduced" equilibrium factor, which is defined as [151]:

$$
F_{\text {Red }}=0.105 f_{218} \mathrm{Po}+0.380 f_{214} \mathrm{Bi}, \mathrm{Po}
$$

where $f_{i}$ is the ratio of the concentrations between the $i$ th progeny and radon.

Employment of three detectors was proposed. The first one was closed inside the diffusion chamber, which measures radon only. Two open detectors were used with different energy windows for separate measurements of ${ }^{218} \mathrm{Po}$ and ${ }^{214} \mathrm{Po}$. The etching conditions applied to the Makrofol detectors for the required energy window were also determined. The reduced equilibrium factor $F_{\text {Red }}$ was then calculated easily. It was shown further that the total equilibrium factor depended on the reduced equilibrium factor in a relatively good manner, as shown in Fig. 49. Comparisons with experimental data obtained from direct active measurements have shown very good agreement.

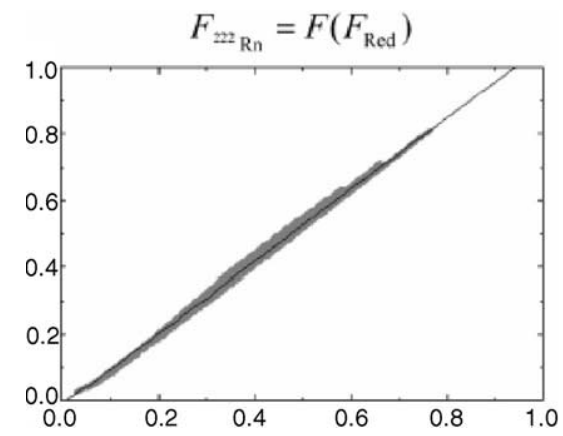

Fig. 49. The ${ }^{222} \mathrm{Rn}$ progeny equilibrium factor expressed as a function of the reduced equilibrium factor, calculated for all possible values for the parameters in the Jacobi model [151]. 


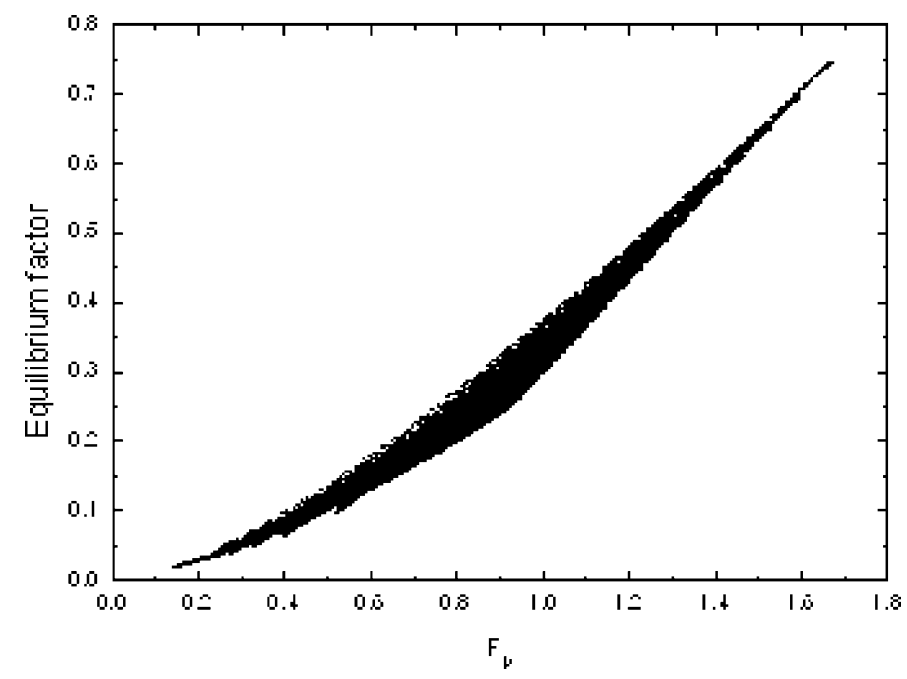

Fig. 50. Dependence of the equilibrium factor $F$ on the proxy equilibrium factor $F_{\mathrm{p}}\left(=F_{1}+F_{3}\right)$ [160].

Along a similar line, Nikezic et al. [160] proposed the measurements of a "proxy" equilibrium factor using a bare LR 115 detector. The partial sensitivities $\rho_{i}$ of the LR 115 detector to ${ }^{222} \mathrm{Rn}$ and its alpha-emitting short-lived progeny, ${ }^{218} \mathrm{Po}$ and ${ }^{214} \mathrm{Po}$, were investigated. They first determined the distributions of lengths of major and minor axes of the perforated alpha tracks in the LR 115 detector produced by ${ }^{222} \mathrm{Rn},{ }^{218} \mathrm{Po}$ and ${ }^{214} \mathrm{Po}$ through Monte Carlo simulations. The distributions determined for different alpha emitters were found to completely overlap with one another. This implied equality of partial sensitivities, $\rho_{i}$, for radon and its progeny, which was also confirmed through analytical considerations. Equality of partial sensitivities made possible convenient measurements of the proxy equilibrium factor $F_{\mathrm{p}}$, which was defined by the authors as $\left(F_{1}+F_{3}\right)$ and was equal to the ratio between the sum of concentrations of the two alpha emitting radon progeny $\left({ }^{218} \mathrm{Po}+{ }^{214} \mathrm{Po}\right)$ to the concentration of radon gas $\left({ }^{222} \mathrm{Rn}\right)$. In particular, the authors found $F_{\mathrm{p}}=\left(\rho / \rho_{i} t C_{0}\right)-1$, where $\rho\left(\operatorname{track} / \mathrm{m}^{2}\right)$ was the total track density on the detector, $t$ was the exposure time and $C_{0}\left(\mathrm{~Bq} / \mathrm{m}^{3}\right)$ was the concentration of ${ }^{222} \mathrm{Rn}$. If $C_{0}$ was known (e.g., from a separate measurement), $F_{\mathrm{p}}$ could be obtained. The proxy equilibrium factor $F_{\mathrm{p}}$ was also found to be well correlated with the equilibrium factor between radon gas and its progeny through the Jacobi room model, which is shown in Fig. 50. This leads to a novel method for long-term determination of the equilibrium factor. This method has also been experimentally tested and calibrated.

\subsection{Retrospective radon dosimetry}

\subsubsection{Basics}

Long-term passive methods based on track detectors can give radon concentration in dwellings for periods usually from 1 month to 1 year. The obtained radon concentrations present the contemporary values. However, to assess the total radon exposure of a person, such measurements might not be satisfactory. For example, a person could have taken some actions that have significantly changed the radon levels, such as home renovation. Radon measurements performed at a particular time might not be able to represent the exposure of a person in the past. However, reconstruction of irradiation history for an individual is usually very difficult, if not impossible. To solve this problem, the so-called retrospective radon measurement was proposed, which are based on measurements of implanted radon progeny in surfaces or volumes of some detectors. 


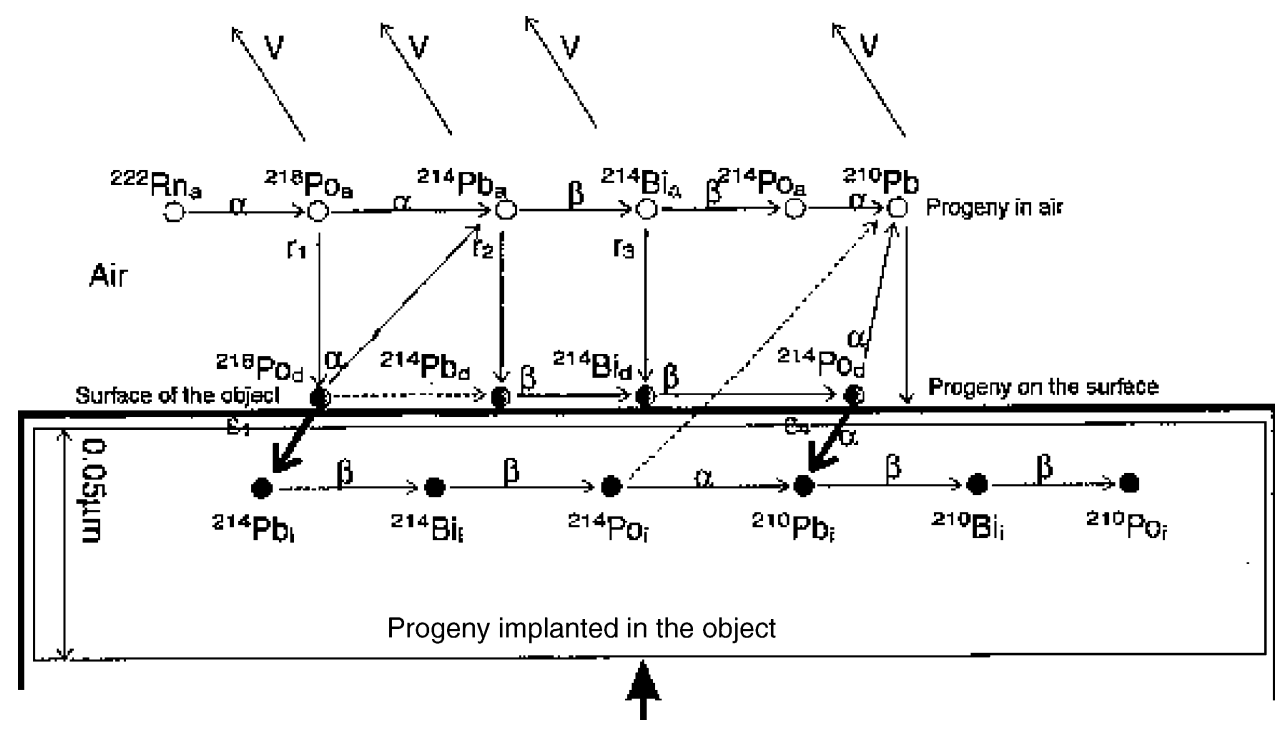

Fig. 51. Radon progeny behavior near a surface [163].

Atoms of short-lived ${ }^{222} \mathrm{Rn}$ progeny deposit on available internal room surfaces. This process is also called plate-out. The plate-out is controlled by air movement and sedimentation, surface to volume ratio and distribution of static electric field in a room. The plate-out has been described in $[161,162]$. Due to recoil after alpha decays of the ${ }^{218} \mathrm{Po}$ and ${ }^{214} \mathrm{Po}$ nuclei, some of the newly formed atoms can be incorporated into the surface. In this way, the subsurface layer is enriched with radon progeny. The activity of the first long-lived ${ }^{222} \mathrm{Rn}$ progeny, ${ }^{210} \mathrm{~Pb}$, implanted into the surface increases with the time of exposure of the object to short-lived ${ }^{222} \mathrm{Rn}$ progeny. The second successor of ${ }^{210} \mathrm{~Pb}$ is the $\alpha$-emitting nuclide ${ }^{210} \mathrm{Po}$, the activity of which in the subsurface layer can be determined by alpha measurements.

The scheme of radon progeny behavior near a surface is given in Fig. 51 [163]. Three modes of radon progeny are shown, namely, progeny in air (denoted by open circles) with indices (a), progeny deposited in the surfaces (half full circles) with indices (s) and finally the progeny incorporated in the surface (full circles) with indices (i). The symbols $r_{1}, r_{2}$ and $r_{3}$ represent the deposition fluxes of ${ }^{218} \mathrm{Po}$, ${ }^{214} \mathrm{~Pb}$ and ${ }^{214} \mathrm{Po}(\mathrm{Bi})$, respectively, which are given in number of deposited atoms $/\left(\mathrm{m}^{2} \mathrm{~s}\right)$, and the symbols $\varepsilon_{1}$ and $\varepsilon_{2}$ are the incorporation probabilities after alpha decays of ${ }^{218} \mathrm{Po}$ and ${ }^{214} \mathrm{Po}$, which are usually taken as 0.5 . The notation $V$ in Fig. 51 means removing of radon progeny from air by ventilation. The type of radioactive decay ( $\alpha$ or $\beta$ decay) was also shown by the symbols $\alpha$ or $\beta$ in Fig. 51. After alpha decay of the deposited ${ }^{218} \mathrm{Po}$, it is possible that newly formed atoms of ${ }^{214} \mathrm{~Pb}$ are returned to the atmosphere. This process is shown in Fig. 51 by the slanted solid line. Furthermore, the hypothetical transition from an incorporated atom to an atom in air after alpha decay of an incorporated ${ }^{214} \mathrm{Po}$ atom is shown as the slanted dashed line. All these processes have been described with a set of differential equations $[163,164]$. The solution of the set of equations in a steady state case leads to the following expression for the ${ }^{210} \mathrm{Po}$ activity $\left(A_{7}\right)$ (seventh radon progeny):

$$
A_{7}=\frac{1}{2}\left(r_{1}+r_{2}+r_{3}\right)\left(1-\mathrm{e}^{-\lambda_{5} t}\right)
$$

where $\lambda_{5}$ is the decay constant of ${ }^{210} \mathrm{~Pb}$ (fifth ${ }^{222} \mathrm{Rn}$ progeny). A similar equation was given by Schmidt and Hamel [165] but other relevant parameters have been used instead of the deposition fluxes. 
In considering the behavior of radon progeny, three modes were taken into account: progeny in air (both attached and unattached to aerosols), deposited and implanted progeny. The Jacobi room model [129] was extended to the long-lived progeny up to ${ }^{210} \mathrm{Po}$. A set of differential equations that describe the behavior of radon and its progeny in all modes was developed. The differential equations related to the short-lived progeny come to linear equations and there is no need to solve a complicated set of interlinked differential equations. However, the differential equations for the long-lived progeny had to be solved because they accumulated over time. Under the assumption of steady state, the following equation was found for the total deposition flux of radon progeny:

$$
r_{\text {tot }}=r_{1}+r_{2}+r_{3}=\frac{V}{S}\left[\lambda_{\mathrm{d}}^{\mathrm{u}}\left(\frac{A_{1}^{\mathrm{u}}}{\lambda_{1}}+\frac{A_{2}^{\mathrm{u}}}{\lambda_{2}}+\frac{A_{3}^{\mathrm{u}}}{\lambda_{3}}\right)+\lambda_{\mathrm{d}}^{\mathrm{a}}\left(\frac{A_{1}^{\mathrm{a}}}{\lambda_{1}}+\frac{A_{2}^{\mathrm{a}}}{\lambda_{2}}+\frac{A_{3}^{\mathrm{a}}}{\lambda_{3}}\right)\right]
$$

where $\lambda_{i}(i=1,2,3)$ are the decay constants of ${ }^{218} \mathrm{Po},{ }^{214} \mathrm{~Pb}$ and ${ }^{214} \mathrm{Bi}$, respectively, $A_{i}^{\mathrm{u}}$ and $A_{i}^{\mathrm{a}}$ are the activity concentrations of the unattached and attached radon progeny, respectively, $\lambda_{\mathrm{d}}^{\mathrm{u}}$ and $\lambda_{\mathrm{d}}^{\mathrm{a}}$ are the deposition rates of the unattached and attached progeny, respectively.

By applying the Jacobi room model [129] for calculation of the deposition flux with the parameters varied in reasonable ranges around the best estimates, and by subsequently fitting the data, the following formula was derived for the cumulative exposure $X$ to radon progeny [164] as:

$$
X=0.08 \frac{A_{7}}{1-\mathrm{e}^{-\lambda_{5} t}} t
$$

where $X$ is given in WLM (working level month), the time $t$ in years and the activity $A_{7}$ of ${ }^{210} \mathrm{Po}$ in Bq/ $\mathrm{m}^{2}$. A linear increase of the cumulative exposure $X$ with the time is observed from Eq. (4.19) since $A_{7}$ is proportional to $1-\mathrm{e}^{-\lambda_{5} t} . A_{7}$ and the expression in the denominator were shown in the expression because the time $t$ and the activity $A_{7}$ are measurable quantities. Eq. (4.19) enables a direct estimation of the effective dose by multiplying with the dose conversion coefficient obtained from epidemiological studies as $5 \mathrm{mSv} / \mathrm{WLM}$.

Other authors related the ${ }^{210}$ Po activity to the total radon exposure rather than to the exposure to radon progeny. For example, Samuelsson [166] has experimentally established that the $\alpha$-activity of ${ }^{210} \mathrm{Po}$ was related to the cumulative ${ }^{222} \mathrm{Rn}$ exposure, and showed that window glasses might serve as cumulative radon dosimeters. Various glass objects were found to be useful for this purpose [167]. For example, the glasses covering wedding photos may serve as good estimator because it is moved with the people. Spectacle glasses or watch glasses may also serve as good individual dosimeters [168]. Other objects like porcelain and ceramic can also be used but problems may arise as a result of the high natural radioactivity level.

Trotti et al. [169] applied the Jacobi model [129] and derived a somewhat different formula for the implanted ${ }^{210}$ Po activity in the surface as:

$$
A_{7} \propto \frac{\lambda_{5}}{S / V} A_{222} \operatorname{Rn}\left(1-\mathrm{e}^{-\lambda_{5} t}\right)
$$

where $A_{222} \mathrm{Rn}$ is the activity concentration of ${ }^{222} \mathrm{Rn}$ in $\mathrm{Bq} / \mathrm{m}^{3}$ in air and $V / S$ is the volume to surface ratio of a room. Eq. (4.20) differs from Eq. (4.17) in the inclusion of deposition fluxes as well as the activity concentration of radon in the former, and both the equations contain the same exponential multiplier.

More recently, Couwels et al. [170] developed the system of differential equations also based on the Jacobi room model, and made extensions to the long-lived progeny. The entire model consisted of 24 differential equations. The approach used was similar to that of Nikezic and Yu $[163,164]$ where the model was split into a steady part related to short-lived progeny as well as a time dependent part for 
long-lived progeny. Couwels et al. [170] solved the full system of equations and found that the implanted ${ }^{210}$ Po activity was a linear function of the average past radon activity.

There were discussions about the influence of dust, glass cleaning and corrosion on ${ }^{210} \mathrm{Po}$ implantation. The influence of dust was studied by Johansson et al. [171]. Roos and Whitlow [172] calculated the distribution of implanted ${ }^{210} \mathrm{~Pb}$ and compared it with the measured one. Similarly to Fig. 51, it was noticed that implantation could occur in single or double steps and these two cases were distinguished in calculations. By analyzing and comparing the experimental and calculated distributions, they showed that diffusion of implanted atoms and cleaning did not significantly affect the total activity of ${ }^{210}$ Po nor its depth distribution.

\subsubsection{Measuring techniques}

To determine the cumulative exposure to radon or radon progeny, one should measure the incorporated ${ }^{210} \mathrm{Po}$ in different objects in a room. The radionuclide ${ }^{210} \mathrm{Po}$ emits alpha particles with an energy of 5.3 MeV. According to Cornelis et al. [173], the implantation depth in glass is up to $0.53 \mu \mathrm{m}$. However, Roos and Whitlow [172] took into account the possibility of double implantation and obtained the maximal implantation depth up to $0.1 \mu \mathrm{m}$. Alpha particles with an energy of $5.3 \mathrm{MeV}$ have a range of about $23 \mu \mathrm{m}$ (calculated using the SRIM program [23]), which is much larger than the maximal implantation depth, and can exit from an object. In this way, the depth distribution of the implanted ${ }^{210}$ Po should not be very important in measurements.

During the last 15 years, several methods have been developed to measure the ${ }^{210}$ Po activity in glass objects by using various techniques of $\alpha$-activity measurements. The implanted activity can be measured by pulse ionizing chamber or with solid-state nuclear track detectors that are placed onto the object for a period of time. Lively and Ney [174] dissolved the surface of a glass object using some acids and measured the ${ }^{210} \mathrm{Po}$ activity in solution. However, this method damaged the surface of the glass and was thus destructive.

Solid-state nuclear track detectors can be used for measurements of the ${ }^{210}$ Po activity in an object. The simplest method is to place a detector directly on a piece of glass, and after exposure for some time to etch and read the detector in the usual way. Calibration of the detector should be carried out by exposing it on glass objects with known surface activity of ${ }^{210} \mathrm{Po}$. A problem here is that some alpha emitters exist naturally in glass objects and these alpha particles can be registered by the detectors. It is therefore necessary to distinguish the implanted ${ }^{210} \mathrm{Po}$ in the surface of the glass object from the alpha emitters in the bulk of glass objects. Different detector designs were used to solve this problem. A few methods were described by Falk et al. [175].

The first one, called the absorber technique, used two CR-39 detectors on the same side of a glass object, one with an absorber between the glass surface and the detector surface, and the other without the absorber. The absorber reduced the number of tracks, by $20 \%$ for ${ }^{210} \mathrm{Po}$ and by $50 \%$ for the background tracks. From this information, one may infer the track numbers due to the implanted ${ }^{210} \mathrm{Po}$ only. However, such a method suffers from the high uncertainty for low surface activities because the difference between two close numbers is required.

The second one is to use two detectors on both sides of the glass object. The difference between the front and the back sides gives the ${ }^{210}$ Po activity. The problem here is that back side (the one facing away from the room volume) is also exposed to some radon progeny.

The third one is the CR-LR difference method $[175,176]$. Two different detectors, CR-39 and LR 115 , are used side by side on the glass object. The CR-39 detector records both the alpha particles emitted from the surface and those emitted in the bulk of the glass object. On the contrary, due to the presence of an upper energy threshold of about $4 \mathrm{MeV}$, the LR 115 detector does not record alpha particles emitted by ${ }^{210} \mathrm{Po}$ incorporated in the surface because their energies will not be decreased 
sufficiently to become smaller than $4 \mathrm{MeV}$. The difference between these two types of detectors will give the required activity of ${ }^{210} \mathrm{Po}$ in the surface.

Falk et al. [175] experimentally tested the techniques mentioned earlier, i.e., the single detector method, using CR-39 on front and back sides of a glass object, CR-LR difference method and others, and concluded that the CR-LR difference method gave correct values for the ${ }^{210}$ Po activity.

Fitzgerald and McLaughlin [177] used an integrated monitor based on two CR-39 detectors. One was put in contact with the glass object recording ${ }^{210} \mathrm{Po}$ from the surface. The other detector was positioned on the opposite side of the holder with two active surfaces, with one surface facing a small gap of about $1 \mathrm{~cm}$ to measure ${ }^{222} \mathrm{Rn}$ only and the other completely open in air to record $\left({ }^{222} \mathrm{Rn}+\right.$ progeny in air + progeny plate-out). According to their calculations, the majority of tracks on the surface of the open CR-39 detector originated just from plate-out.

Meesen et al. [178] used Makrofol polycarbonate detectors which had an optimum sensitivity for alpha particles with energies between 0.3 and $2.0 \mathrm{MeV}$. Because of this energy window, it was necessary to lower the energy of the alpha particles emitted by ${ }^{210} \mathrm{Po}(5.31 \mathrm{MeV})$. For this purpose, a Mylar layer with a thickness of $23 \mu \mathrm{m}$ was placed between the detector material and the glass plate. The combination of the detector and Mylar film was mounted on the glass object by means of an adhesive tape, and was referred to as the "210 Po detector".

In contrary to the previous approaches where the gross alpha activity was measured, Trotti et al. [169] employed alpha spectrometry on the CR-39 detector to obtain the ${ }^{210}$ Po activity implanted in the surface. This technique was tedious but the use of an automatic readout device might facilitate it.

\subsubsection{Volumetric trap of ${ }^{210} \mathrm{Po}$}

McLaughlin [179] summarized different approaches to assess the long-term exposure to radon and its progeny. In addition to the measurements of ${ }^{210} \mathrm{Po}$ in the surface, one more technique called the volume trap was discussed. These volume traps are spongy and porous materials in dwellings, and radon may diffuse into and decay within the internal pores so that the ${ }^{210} \mathrm{~Pb}$ activity will build up in the pores [180]. The ${ }^{210} \mathrm{~Pb}$ activity can be measured using radiochemical methods. While the activity of ${ }^{210} \mathrm{Po}$ implanted in the surface of objects depends on parameters like the aerosol size distribution, aerosol concentration, characteristics of air movement in the room, and room geometry, etc.; the volumetric concentration of long-lived radon progeny does not depend on any of these factors and is a direct indicator of the radon activity only. In this way, the volumetric activity of long-lived radon progeny may be a better estimator of the average past radon exposure.

\subsubsection{Applications of retrospective radon dosimetry}

There were many papers which described the application of retrospective radon dosimetry using solid-state nuclear track detectors. McLaughlin [181] described the procedures as well as the criteria for choosing of the objects to be investigated for retrospective dosimetry. Falk et al. [176] compared the average radon levels estimated using the CR-LR difference method with the contemporary measurements, and found a correlation given by $r^{2}=0.53$. Birovljev et al. [182] performed retrospective radon dosimetry based on the ${ }^{210}$ Po incorporated in the surface in Norwegian dwellings. They also compared the results with contemporary measurements and found a larger correlation coefficient of about 0.877 . Retrospective dosimetry was also applied in combination with contemporary measurements in two stable rural communities in Yugoslavia [183]. Paridaens et al. [184] used the volumetric trap method for the samples from several European countries and emphasized that polyester materials were the best for the volumetric method for retrospective dosimetry.

As a conclusion of this section, we should emphasize that there is a hope that improvement of the retrospective dosimetry methods will give a better estimation of the past radon exposure. The latter 
will be helpful in epidemiological and case control studies to establish the relation between radon exposure and the lung cancer risk.

\section{Applications}

Solid-state nuclear track detectors have found many applications in various fields of physics and other branches of science. Apart from measurements of indoor radon and progeny concentrations, which have been discussed in more details in previous sections, there are many other applications. It is not our intention here to describe all possible usages of solid-state nuclear track detectors in details. Instead, we will only pick some of the more common applications and then supplement with very brief comments, where appropriate. For more information about different applications, the reader is referred to books and conference proceedings [4-12].

Applications related to radon measurements:

1. Radon measurements in tap water, natural water and soil: This kind of measurements are useful in uranium and thorium prospecting. It is also important to identify the radon rich areas. Knowledge of the radon levels in soil is important for classifying different areas for construction purposes and for planning of new buildings [185-193].

2. Radon emanation and exhalation from soil and building materials: It is useful to characterize building materials and soils as radon sources [194-200].

3. Radon monitoring in mines and other underground places: This is an important issue for radiological protection of miners, as well as in epidemiological studies involving miners [201208].

4. Separation of thoron from radon: This is usually achieved based on the distance, because thoron is relatively very short-lived and cannot diffuse too far from the place of origin [209-211].

5. Application in earth sciences and radon measurements in caves [212-214].

6. Earthquake prediction: Radon concentrations in underground water and deep wells have been observed to increase significantly before earthquake [215-222].

7. Volcanic studies: The radon flux increases before volcanic eruption [223].

Applications other than radon measurements:

1. In many areas of nuclear physics: For identification of products from some reactions in high and low energy physics.

2. Producing micro filters [224].

3. Neutron dosimetry and reactor physics: After collision of a neutron with a proton in the detector material, a recoil proton is formed which may leave latent track in the detector. This is the basis for neutron measurements with the track detectors [225-230].

4. Neutrino measurements: Neutrinos are neutral particles with very small mass created in beta decays, and are very difficult for detection. The solar neutrinos and other neutrinos are readily measured by a stack of large detectors [231].

5. Dose measurements in space and aircrafts.

6. Fission track dating. Spontaneous fission of ${ }^{238} \mathrm{U}$ occurs with a small probability. Some natural minerals contain certain amounts of ${ }^{238} U$ and can store the tracks produced by fission. As the number of tracks grows with the age of the minerals, the latter can be determined based on the known ${ }^{238} \mathrm{U}$ concentrations [232,233]. 
7. High energy and cosmic ray physics: Cosmic rays can produce tracks in natural minerals with dominant orientations. The tracks may be used for particle identification and for studies of the cosmic rays. Tracks of cosmic rays are also observed in meteorites [234].

8. Search for magnetic monopole: The magnetic monopole is a particle predicted theoretically [235] to provide some basic understanding in physics. However, all efforts in this direction did not prove the existence of magnetic monopole.

9. Medicine and biology: Novel applications of track detectors have been found in the investigation of the effects of alpha particles on living cells which are cultured on the detectors themselves [236239].

\section{Acknowledgements}

This work is supported by the grants CityU 102803 and CityU 103204 from the Research Grants Council of Hong Kong.

\section{References}

[1] D.A. Young, Nature 182 (1958) 375.

[2] E.C.H. Silk, R.S. Barnes, Philos. Mag. 4 (1959) 970.

[3] R.L. Fleischer, P.B. Price, R.M. Walker, Annu. Rev. Nucl. Sci. 15 (1965) 1.

[4] R.L. Fleischer, P.B. Price, R.M. Walker, Nuclear Tracks in Solids, University of California Press, Berkley, 1975.

[5] S.A. Durrani, R.K. Bull, Solid State Nuclear Track Detection. Principles, Methods and Applications, Pergamon Press, 1987.

[6] S.A. Durrani, R. Ilic, Radon Measurements by Etched Track Detectors: Applications in Radiation Protection, Earth Sciences and the Environment, World Scientific, Singapore, 1997.

[7] R.L. Fleischer, Tracks to Innovation: Nuclear Tracks in Science and Technology, Springer, 1998.

[8] K.K. Dwivedi, R. Ilic, P. Vater, Radiat. Meas. 36 (2003).

[9] R. Ilic, I. Jencic, P. Vater, Radiat. Meas. 34 (2001).

[10] A. Chambaudet, M. Fromm, R. I1ic, P. Vater, C. Dubois, M. Rebetez, Radiat. Meas. 31 (1999).

[11] R. Ilic, P. Vater, M.A. Kenawy, Radiat. Meas. 28 (1997).

[12] V.P. Perelygin, P. Vater, R. Ilic, S.A. Durrani, Radiat. Meas. 25 (1995).

[13] B.G. Cartwright, E.K. Shirk, P.B. Price, Nucl. Instrum. Methods 153 (1978) 457.

[14] R.L. Fleischer, P.B. Price, R.M. Walker, J. Appl. Phys. 36 (1965) 3645.

[15] F. Seitz, J.S. Koehler, Solid State Phys. 2 (1956) 305.

[16] L.T. Chadderton, H.M. Montagu-Pollock, Proc. R. Soc. A 274 (1963) 239.

[17] R. Barillon, M. Fromm, A. Chambaudet, R. Katz, J.P. Stoquert, A. Pape, Radiat. Meas. 31 (1999) 71.

[18] L.T. Chadderton, Radiat. Meas. 36 (2003) 13.

[19] J. Turner, Health Phys. 86 (2004) 228.

[20] N. Bohr, Philos. Mag. 15 (1913) 10.

[21] H. Bethe, Ann. Phys. 5 (1930) 325 (in German).

[22] F. Bloch, Zeits Phys. 81 (1933) 363 (in German).

[23] J.F. Ziegler, J.P. Biersack, U. Littmark, The Stopping and Ranges of Ions in Solids, Pergamon Press, Oxford, 1985, the code is available on http://www.srim.org.

[24] P.R. Henke, E. Benton, Nucl. Instrum. Methods 97 (1971) 483.

[25] G. Somogyi, A.S. Szalay, Nucl. Instrum. Methods 109 (1973) 211.

[26] G.H. Paretzke, E. Benton, P.R. Henke, Nucl. Instrum. Methods 108 (1973) 73.

[27] G. Somogyi, Nuclear Instrum. Methods 173 (1980) 21.

[28] M. Fromm, P. Meyer, A. Chambaudet, Nucl. Instrum. Methods Phys. Res. B 107 (1996) 337.

[29] R. Barillon, M. Fromm, A. Chambaudet, H. Marah, A. Sabir, Radiat. Meas. 28 (1997) 619.

[30] V. Ditlov, Radiat. Meas. 25 (1995) 89.

[31] D. Nikezic, D. Kostic, Radiat. Meas. 28 (1997) 185. 
[32] D. Nikezic, Radiat. Meas. 32 (2000) 277.

[33] D. Nikezic, K.N. Yu, Radiat. Meas. 37 (2003) 39.

[34] A.P. Fews, D.L. Henshaw, Nucl. Instrum. Methods 197 (1982) 517.

[35] B. Dorschel, D. Hermsdorf, U. Reichelt, S. Starke, Radiat. Meas. 37 (2003) 573.

[36] D. Nikezic, Nucl. Instrum. Methods Phys. Res. A 344 (1994) 406.

[37] D. Nikezic, K.N. Yu, http://www.cityu.edu.hk/ap/nru/nrures_t.htm.

[38] P.G. Green, A.G. Ramli, S.A.R. Al-Najjar, F. Abu-Jarad, S.A. Durrani, Nucl. Instrum. Methods 203 (1982) 551.

[39] Z.S. Kocsis, K.K. Dwivedi, R. Brandt, Radiat. Meas. 28 (1997) 177.

[40] C.W.Y. Yip, J.P.Y. Ho, D. Nikezic, K.N. Yu, Radiat. Meas. 36 (2003) 161.

[41] D.T. Clark, P.J. Stephenson, Polym. Degrad. Stab. 4 (1982) 185.

[42] A.H.K. Fowler, H.S. Munro, D.T. Clark, Polym. Degrad. Stab. 11 (1985) 287.

[43] F.M.F. Ng, C.W.Y. Yip, J.P.Y. Ho, D. Nikezic, K.N. Yu, Radiat. Meas. 38 (2004) 1.

[44] K.N. Yu, F.M.F. Ng, Nucl. Instrum. Methods Phys. Res. B, in press.

[45] M. Fujii, R. Yokota, Y. Atarashi, H. Hasegawa, Radiat. Meas. 19 (1991) 171.

[46] J.H. Adams, in: Proceedings of the 10th Conference on SSNTD, Lyon, 2-6 July 1979, p. 223.

[47] P.H. Fowler, S. Amin, V.M. Clapham, D.L. Henshaw, in: Proceedings of the 10th Conference on SSNTD, Lyon, 2-6 July 1979, p. 239.

[48] T.A. Gruhn, W.K. Li, E.V. Benton, R.M. Cassou, C.S. Johnson, in: Proceedings of the 10th Conference on SSNTD, Lyon, 2-6 July 1979, p. 291.

[49] S.A.R. Al-Najjar, R.K. Bull, S.A. Durrani, in: Proceedings of the 10th Conference on SSNTD, Lyon, 2-6 July 1979 , p. 323.

[50] G. Somogyi, I. Hunyadi, in: Proceedings of the 10th Conference on SSNTD, Lyon, 2-6 July 1979, p. 443.

[51] F. Ansari, Matiullah, N.A. Javaid, E.U. Khan, M. Fujii, Radiat. Meas. 19 (1991) 139.

[52] M. Fromm, F. Membrey, A. Chambaudet, R. Saouli, Radiat. Meas. 19 (1991) 163.

[53] E.M. Awad, H.M. El-Samman, Radiat. Meas. 31 (1999) 109.

[54] A. Szydlowski, T. Czyzewski, M. Jaskola, M. Sadowski, A. Korman, J. Kedzierski, W. Kretschmer, Radiat. Meas. 31 (1999) 257.

[55] A. Kumar, R. Prasad, Radiat. Meas. 31 (1999) 227.

[56] S. Cecchini, G. Giacomelli, M. Giorgini, L. Patrizii, P. Serra, Radiat. Meas. 34 (2001) 55.

[57] M. Siems, K. Freyer, H.-C. Treutler, G. Jonsson, W. Enge, Radiat. Meas. 34 (2001) 81.

[58] F. Abu-Jarad, A.M. Hala, M. Farhat, M. Islam, Radiat. Meas. 28 (1997) 111.

[59] D. Sinha, S. Ghosh, A. Srivastava, V.G. Dedgaonkar, K.K. Dwiwedi, Radiat. Meas. 28 (1997) 145.

[60] T. Yamauchi, T. Taniguchi, K. Oda, T. Ikeda, Y. Honda, S. Tagawa, Radiat. Meas. 31 (1999) 121.

[61] T. Yamauchi, H. Ichijo, K. Oda, Radiat. Meas. 34 (2001) 85.

[62] Fazal-ur-Rehman, F. Abu-Jarad, M.I. Al-Jarallah, M. Farhat, Radiat. Meas. 34 (2001) 617.

[63] A. Szydlowski, A. Banaszak, I. Fijal, M. Jaskola, A. Korman, M. Sadowski, Z. Zimek, Radiat. Meas. 36 (2003) 111.

[64] R. Mishra, S.P. Tripathy, K.K. Dwivedi, D.T. Khathing, S. Ghosh, M. Muler, D. Fink, Radiat. Meas. 34 (2001) 95.

[65] F. Abu-Jarad, M. El Hadidy, M.I. Al-Jarallah, Radiat. Meas. 28 (1997) 409.

[66] F. Abu-Jarad, M.A. Islam, I. Abu-Abdoun, M.A. Khan, Radiat. Meas. 19 (1991) 135.

[67] K.K. Dwivedi, S. Ghosh, D. Fink, R. Mishra, S.P. Tripathy, A. Kulshreshta, D.T. Khathing, Radiat. Meas. 31 (1999) 127.

[68] S.P. Tripathy, R. Mishra, K.K. Dwivedi, D.T. Khathing, S. Ghosh, D. Fink, Radiat. Meas. 36 (2003) 107.

[69] S. Prasher, S. Singh, Radiat. Meas. 36 (2003) 105.

[70] D. Nikezic, A. Janicijevic, Appl. Radiat. Isot. 57 (2002) 275.

[71] N. Yasuda, K. Uchikawa, K. Amemiya, N. Watanabe, H. Takahashi, M. Nakazawa, M. Yamamoto, K. Ogura, Radiat. Meas. 34 (2001) 45.

[72] J.P.Y. Ho, C.W.Y. Yip, D. Nikezic, K.N. Yu, Radiat. Meas. 36 (2003) 141.

[73] C.W.Y. Yip, J.P.Y. Ho, V.S.Y. Koo, D. Nikezic, K.N. Yu, Radiat. Meas. 37 (2003) 197.

[74] C.W.Y. Yip, J.P.Y. Ho, D. Nikezic, K.N. Yu, Radiat. Meas. 36 (2003) 245.

[75] J.P.Y. Ho, C.W.Y. Yip, D. Nikezic, K.N. Yu, Radiat. Meas. 36 (2003) 155.

[76] J.B. Vukovic, R. Atanasijevic, Radiat. Meas. 25 (1995) 745.

[77] J.P.Y. Ho, C.W.Y. Yip, V.S.Y. Koo, D. Nikezic, K.N. Yu, Radiat. Meas. 35 (2002) 571.

[78] D. Nikezic, J.P.Y. Ho, C.W.Y. Yip, V.S.Y. Koo, K.N. Yu, Nucl. Instrum. Methods Phys. Res. B 197 (2002) 293.

[79] C. Vazquez-Lopez, R. Fragoso, J.I. Golzarri, F. Castillo-Mejia, M. Fujii, G. Espinosa, Radiat. Meas. 34 (2001) 189.

[80] N. Yasuda, M. Yamamoto, K. Amemiya, H. Takahashi, A. Kyan, K. Ogura, Radiat. Meas. 31 (1999) 203. 
[81] F. Palmino, D. Klein, J.C. Labrune, Radiat. Meas. 31 (1999) 209.

[82] D. Nikezic, K.N. Yu, Radiat. Meas. 37 (2003) 39.

[83] D. Nikezic, K.N. Yu, Radiat. Meas. 37 (2003) 595.

[84] D. Nikezic, K.N. Yu, Nucl. Instrum. Methods Phys. Res. B 196 (2002) 105.

[85] G. Somogyi, Gy. Almasi, in: Proceedings of the 10th Conference on SSNTD, Lyon, 2-6 July 1979, p. 257.

[86] G. Somogyi, I. Hunyadi, in: Proceedings of the 10th Conference on SSNTD, Lyon, 2-6 July 1979, p. 443.

[87] D.L. Henshaw, A.P. Fews, D.J. Webster, in: Proceedings of the 10th Conference on SSNTD, Lyon, 2-6 July 1979 , p. 649.

[88] G. Giacomelli, L. Patrizii, V. Popa, P. Serra, V. Togo, Radiat. Meas. 28 (1997) 217.

[89] S. Singh, L. Singh, J. Sing, H.S. Virk, Radiat. Meas. 19 (1991) 121.

[90] M. Yamamoto, N. Yasuda, Y. Kaizuka, M. Yamagishi, T. Kanai, N. Ishugure, A. Furukawa, M. Kurano, N. Miyhara, M. Nakazawa, T. Doke, K. Ogura, Radiat. Meas. 28 (1997) 227.

[91] T. Doke, T. Hayashi, M. Kobayashi, A. Watanabe, Radiat. Meas. 28 (1997) 445.

[92] G. Meesen, P. Van Oostveldt, Radiat. Meas. 28 (1997) 845.

[93] J. Jakes, P. Gais, J. Voigt, Radiat. Meas. 28 (1997) 853.

[94] B. Dorschel, A. Guhr, M. Mansy, P. Schmidt, G. Streubel, Radiat. Meas. 19 (1991) 155.

[95] L. Borgonovo, G. Saint-Martin, O.A. Bernaola, I. Nemirovsky, W. Kirschbaum, Radiat. Meas. 28 (1997) 821.

[96] K.N. Yu, F.M.F. Ng, J.P.Y. Ho, C.W.Y. Yip, D. Nikezic, Radiat. Prot. Dosim., in press.

[97] B. Dörschel, H. Hartmann, K. Kadner, P. Rössler, Radiat. Meas. 25 (1995) 157.

[98] M. Fujii, Y. Hatano, A. Osawa, T. Saito, K. Yamamoto, T. Hasebe, T. Nakamura, H. Sasaki, T. Yanagita, M. Aglietta, S. Verneto, A. Castellina, W. Fulgione, O. Saavedra, G.C. Trinchero, Radiat. Meas. 31 (1999) 591.

[99] P.K. Diwan, V. Sharma, S.K. Sharma, S. Kumar, Radiat. Meas. 36 (2003) 89.

[100] T. Yamauchi, T. Taniguchi, K. Oda, Radiat. Meas. 31 (1999) 261.

[101] P. Gais, J. Jakes, H. Schraube, Radiat. Meas. 25 (1995) 767.

[102] F. Vaginay, M. Fromm, D. Pusset, G. Meesen, A. Chambaudet, A. Poffijn, Radiat. Meas. 34 (2001) 123.

[103] G. Meesen, A. Poffijn, Radiat. Meas. 34 (2001) 161.

[104] C. Brun, M. Fromm, M. Jouffroy, P. Meyer, J.E. Groetz, F. Abel, A. Chambaudet, B. Dorschel, D. Hermsdorf, R. Bretschneider, K. Kadner, H. Kuhne, Radiat. Meas. 31 (1999) 89.

[105] D.L. Henshaw, S. Amin, V.M. Clapham, P.H. Fowler, D.J. Webster, A. Thompson, D.O. Sullivan, in: Proceedings of the 11th Conference on SSNTD, Bristol, 7-12 September 1981, p. 115.

[106] P. Baiocchi, S. Cecchini, H. Dekhissi, V. Garutti, G. Giacomelli, G.G. Giani, E. Katsavounidis, G. Iori, L. Patrizii, V. Popa, P. Serra, V. Togo, U. Valdre, E. Vilela, Radiat. Meas. 25 (1995) 145.

[107] F. Petersen, W. Enge, Radiat. Meas. 25 (1995) 43.

[108] B. Dorschel, D. Hermsdorf, K. Kadner, S. Starke, Radiat. Meas. 35 (2002) 287.

[109] B. Dorschel, D. Hermsdorf, K. Kadner, S. Starke, Radiat. Meas. 35 (2002) 293.

[110] B. Dorschel, D. Hermsdorf, S. Starke, Radiat. Meas. 37 (2003) 583.

[111] M. Fromm, F. Vaginay, G. Meesen, A. Chambaudet, A. Poffijn, Radiat. Meas. 36 (2003) 93.

[112] V.A. Nikolaev, R. Ilic, Radiat. Meas. 30 (1999) 1.

[113] K.N. Yu, E.C.M. Young, M.J. Stokes, Z.J. Guan, K.W. Cho, Health Phys. 73 (1997) 373.

[114] K.N. Yu, T. Cheung, Z.J. Guan, E.C.M. Young, W.N. Mui, Y.Y. Wong, J. Environ. Radioact. 45 (1999) 291.

[115] K.N. Yu, T. Cheung, Z.J. Guan, W.N. Mui, Y.T. Ng, J. Environ. Radioact. 48 (2000) 211.

[116] K.N. Yu, Z.J. Guan, M.J. Stokes, E.C.M. Young, J. Environ. Radioact. 17 (1992) 31.

[117] A.E. Liehu, I. Makelainen, M. Valkiainen, K. Winqvist, in: P.H. Fowler, V.M. Clapham (Eds.), in: Proceedings of the 11th International Conference on Solid State Nuclear Track Detectors, Bristol, Pergamon Press, Oxford, 1981 , p. 557.

[118] D. Nikezic, K.N. Yu, Nucl. Instrum. Methods Phys. Res. A 419 (1998) 175.

[119] D. Nikezic, K.N. Yu, Health Phys. 78 (2000) 414.

[120] V.S.Y. Koo, C.W.Y. Yip, J.P.Y. Ho, D. Nikezic, K.N. Yu, Appl. Radiat. Isot. 56 (2002) 953.

[121] G. Sciocchetti, G. Cotellessa, E. Soldano, M. Pagliari, Radiat. Meas. 36 (2003) 199.

[122] I. Csige, S. Csegzi, Radiat. Meas. 34 (2001) 437.

[123] R.S. Neman, I.D. Schmitman, J.C.N. Hadler, P.J. Iunes, S.R. Paulo, S. Guedes, Radiat. Meas. 34 (2001) 145.

[124] M. Calamosca, S. Penzo, G. Gualdrini, Radiat. Meas. 36 (2003) 221.

[125] S. Somogyi, B. Paripas, Z. Varga, Nucl. Tracks Radiat. Meas. 8 (1984) 423.

[126] D. Nikezic, K.N. Yu, Nucl. Instrum. Methods Phys. Res. A 450 (2000) 568.

[127] D. Nikezic, F.M.F. Ng, K.N. Yu, Nucl. Instrum. Methods Phys. Res. B 217 (2004) 637.

[128] J.P. McLaughlin, B. Fitzgerald, Radiat. Prot. Dosim. 56 (1994) 241. 
[129] W. Jacobi, Health Phys. 22 (1972) 441.

[130] D. Pressyanov, I. Rusinov, G. Simeonov, Nucl. Instrum. Methods Phys. Res. A 435 (1999) 509.

[131] D. Nikezic, P. Markovic, D. Bek Uzarov, Health Phys. 64 (1993) 628.

[132] V.S.Y. Koo, C.W.Y. Yip, J.P.Y. Ho, D. Nikezic, K.N. Yu, Nucl. Instrum. Methods Phys. Res. A 491 (2002) 470.

[133] V.S.Y. Koo, C.W.Y. Yip, J.P.Y. Ho, D. Nikezic, K.N. Yu, Appl. Radiat. Isot. 59 (2003) 49.

[134] D. Nikezic, C. Baixeras, Nucl. Instrum. Methods Phys. Res. A 364 (1995) 531.

[135] D. Nikezic, K.N. Yu, Nucl. Instrum. Methods Phys. Res. A 437 (1999) 531.

[136] J.C.H. Miles, in: S.A. Durrani, R. Ilic (Eds.), Radon Measurements by Etched Track Detectors, World Scientific, Singapore, 1997, p. 143.

[137] H.A. Khan, I.E. Qureshi, M. Tufail, Radiat. Prot. Dosim. 46 (1993) 149.

[138] R.L. Fleischer, A. Mogro-Compero, J. Geophys. Res. 83 (B7) (1978) 3539.

[139] F. Bagnoli, F. Bochicchio, S. Bucci, D. Marocco, Radiat. Meas. 34 (2001) 207.

[140] L. Tommasino, Nucl. Tracks Radiat. Meas. 17 (1990) 43.

[141] D. Marocco, F. Bochicchio, Radiat. Meas. 34 (2001) 509.

[142] B. Dorschel, D. Hermsdorf, U. Reichelt, Radiat. Meas. 35 (2002) 189.

[143] A. Damkjaer, Nucl. Tracks Radiat. Meas. 12 (1986) 295.

[144] D. Nikezic, P. Markovic, D. Bek Uzarov, Health Phys. 62 (1992) 239.

[145] O. Sima, Radiat. Meas. 34 (2001) 181.

[146] R. Andriamanatena, G.U. Bacmeister, K. Freyer, R. Ghose, G. Jonsson, T. Kleis, H.C. Treutler, W. Enge, Radiat. Meas. 28 (1997) 657.

[147] K. Amgarou, Ll. Font, C. Domingo, F. Fernandez, C. Baixeras, Radiat. Meas. 34 (2001) 139.

[148] D. Nikezic, D. Velickovic, Radiat. Meas. 23 (1994) 219.

[149] S. Djeffal, M. Allab, Radiat. Prot. Dosim. 47 (1993) 611.

[150] International Commission on Radiological Protection (ICRP), Protection against radon-222 at home and at work, ICRP Publication 65, Ann. ICRP 23 (2), 1994.

[151] K. Amgarou, Ll. Font, C. Baixeras, Nucl. Instrum. Methods Phys. Res. A 506 (2003) 186.

[152] A.L. Frank, E.V. Benton, Nucl. Track Detect. 1 (1977) 149.

[153] R.L. Fleischer, Health Phys. 47 (1984) 263.

[154] J. Planinic, Z. Faj, Health Phys. 59 (1990) 349.

[155] B. Dorschel, 1992, personal communication.

[156] B. Dorschel, E. Piesch, Radiat. Prot. Dosim. 48 (1993) 145.

[157] D. Nikezic, C. Baixeras, Radiat. Meas. 26 (1996) 203.

[158] D. Nikezic, A. Janicijevic, 1997, unpublished work.

[159] D. Nikezic, K.N. Yu, Nucl. Instrum. Methods Phys. Res. B 187 (2002) 492.

[160] D. Nikezic, F.M.F. Ng, K.N. Yu, Appl. Radiat. Isot., in press.

[161] A.C. George, E.O. Knutson, K.W. Tu, Health Phys. 45 (1983) 439.

[162] E.O. Knutson, A.C. George, J.J. Frey, B.R. Koh, Health Phys. 45 (1983) 445.

[163] D. Nikezic, K.N. Yu, Radiat. Prot. Dosim. 82 (1999) 141.

[164] D. Nikezic, K.N. Yu, J. Environ. Radioact. 47 (2000) 45.

[165] V. Schmidt, P. Hamel, Sci. Tot. Environ. 272 (2001) 189.

[166] C. Samuelsson, Nature 344 (1988) 338.

[167] J.A. Mahaffey, M.A. Parkhurst, A.C. James, F.T. Cross, M.C.R. Alavanja, J.D. Boice, S. Ezrine, P. Henderson, R.C. Brownson, Health Phys. 64 (1993) 381.

[168] S.A. Hadley, N.R. Meyer, R.L. Fleischer, A. Cavallo, Health Phys. 79 (2000) 242.

[169] F. Trotti, P. Mozzo, A. Temporin, M. Lanciai, F. Predicatori, F. Righetti, A. Tacconi, Environ. Int. 22 (Suppl. 1) (1996) S863.

[170] P. Couwels, A. Poffijn, W. Mondelaers, P. Lahorte, B. Masschaele, G. Meesen, A. Van Deynse, Health Phys. 79 (2000) 389.

[171] L. Johansson, M. Wolff, C. Samuelsson, Radiat. Prot. Dosim. 56 (1994) 141.

[172] B. Roos, H.J. Whitlow, Health Phys. 84 (2003) 72.

[173] J. Cornelis, H. Vanmarcke, C. Landsheere, A. Poffijn, Health Phys. 65 (1993) 414.

[174] R.S. Lively, E.P. Ney, Health Phys. 52 (1987) 411.

[175] R. Falk, H. Mellander, L. Nyblom, I. Ostergren, Environ. Int. 22 (Suppl. 1) (1996) S857.

[176] R. Falk, K. Almren, I. Ostergren, Sci. Tot. Environ. 272 (2001) 61.

[177] B. Fitzgerald, J.P. McLaughlin, Environ. Int. 22 (Suppl. 1) (1996) S845. 
[178] G. Meesen, A. Poffijn, J. Uyttenhove, J. Buysse, Radiat. Meas. 25 (1995) 591.

[179] J.P. McLaughlin, Sci. Tot. Environ. 272 (2001) 53.

[180] C. Samuelsson, L. Johansson, Radiat. Protect. Dosim. 56 (1994) 123.

[181] J.P. McLaughlin, Radiat. Prot. Dosim 78 (1998) 1.

[182] A. Birovljev, R. Falk, C. Walsh, F. Bissolo, F. Trotti, J.P. McLaughlin, J. Paridaens, H. Vanmarcke, Sci. Tot. Environ. 272 (2001) 181.

[183] Z.S. Zunic, J.P. McLaughlin, C. Walsh, A. Birovljev, S.E. Simopoulos, B. Jakupi, V. Gordanic, M. Demajo, F. Trotti, R. Falk, H. Vanmarcke, J. Paridaens, K. Fujimoto, Sci. Tot. Environ. 272 (2001) 253.

[184] J. Paridaens, H. Vanmarcke, Z. Zunic, J.P. McLaughlin, Sci. Tot. Environ. 272 (2001) 295.

[185] K. Kristiansson, L. Malmqvist, Geoexploration 22 (1984) 17.

[186] C. Papastefanou, J Environ. Radioact. 63 (2002) 271.

[187] Z.A. Tayyeb, A.R. Kinsara, S.M. Farid, J. Environ. Radioact. 38 (1998) 97.

[188] A. Chavez, M. Monnin, N. Segovia, J.L. Seidel, P. Peña, A. Moreno, M. Balcazar, Radiat. Meas. 27 (1997) 587.

[189] S.M. Farid, J. Environ. Radioact. 34 (1997) 29.

[190] A. Louizi, D. Nikolopoulos, V. Koukouliou, K. Kehagia, Radiat. Prot. Dosim. 106 (2003) 219.

[191] G. Jonsson, Radiat. Meas. 34 (2001) 415.

[192] R. Lehmann, J. Kemski, A. Siehl, R. Stegemann, M. Valdivia-Manchego, Int. Cong. Ser. 1225 (2002) 55.

[193] M. Karpinska, S. Wolkowicz, Z. Mnich, M. Zalewski, K. Mamont-Ciesla, J. Kapala, J. Environ. Radioact. 61 (2002) 149.

[194] C. Baixeras, B. Erlandsson, Ll. Font, G. Jönsson, Radiat. Meas. 34 (2001) 441.

[195] H.U. Johner, H. Surbeck, Sci. Tot. Environ. 272 (2001) 337.

[196] A.K. Burke, A.C. Stancato, V.A. Paulon, S. Guedes, J.C. Hadler, Build. Environ. 38 (2003) 1291.

[197] Fazal-ur-Rehman, M.I. Al Jarallah, M.S. Musazay, F. Abu-Jarad, Appl. Radiat. Isot. 59 (2003) 353.

[198] D.K. Sharma, A. Kumar, M. Kumar, S. Singh, Radiat. Meas. 36 (2003) 363.

[199] K.M. Abumurad, M. Al-Tamimi, Radiat. Meas. 34 (2001) 423.

[200] A.F. Hafez, A.S. Hussein, N.M. Rasheed, Appl. Radiat. Isot. 54 (2001) 291.

[201] A.A. Qureshi, D.M. Kakar, M. Akram, N.U. Khattak, M. Tufail, K. Mehmood, K. Jamil, H.A. Khan, J. Environ. Radiaoact. 48 (2000) 203.

[202] L. Tomásek, T. Müller, E. Kunz, A. Heribanová, J. Matzner, V. Placek, I. Burian, J. Holecek, Int. Cong. Ser. 1225 (2002) 239.

[203] L. Tomásek, E. Kunz, T. Müller, J. Hulka, A. Heribanová, J. Matzner, V. Placek, I. Burian, J. Holecek, Sci. Tot. Environ. 272 (2001) 43.

[204] K. Jamil, S. Ali, J. Environ. Radioact. 54 (2001) 415.

[205] J.H. Lubin, J.D. Boice Jr., J. Nat. Cancer Inst. 89 (1997) 49.

[206] G. Yener, E. Kucuktas, Analyst 123 (1998) 31.

[207] B. Jakupi, G. Krstic, M. Tonic, R. Ilic, Radiat. Meas. 28 (1997) 691.

[208] K. Wiegand, S.P. Dunne, Ann. Occup. Hyg. 40 (1996) 569.

[209] J. Porstendörfer, J. Aerosol Sci. 25 (1994) 219.

[210] P.C. Deka, S. Sarkar, B. Bhattacharjee, T.D. Goswami, B.K. Sarma, T.V. Ramachandran, Radiat. Meas. 36 (2003) 431.

[211] N. Sharma, H.S. Virk, Radiat. Meas. 34 (2001) 467.

[212] J. Hakl, I. Hunyadi, I. Csige, G. Geczy, L. Lenart, A. Varhegyi, Radiat. Meas. 28 (1997) 675.

[213] J. Hakl, I. Csige, I. Hunyadi, A. Varhegyi, G. Geczy, Environ. Int. 22 (Suppl. 1) (1996) 433.

[214] L.S. Bohus, E.D. Greaves, J. Palfalvi, F. Urbani, G. Merlo, Radiat. Meas. 28 (1997) 725.

[215] V.I. Ulumov, A.I. Zakharova, N.V. Ulomova, Akad. Nauk. SSSR Geophys. 177 (1967) 567.

[216] V.I. Ulumov, B.Z. Marashev, Dokl. Akad. Sci. USSR, Earth Sci. Sect. 176 (1968) 319.

[217] R.L. Fleischer, in: S.A. Durrani, R. Ilic (Eds.), Radon Measurements by Etched Track Detectors: Application in Radiation Protection Earth Sciences and the Environment, World Scientific, Singapore, 1997, p. 285.

[218] C.Y. King, in: Proceedings of the International Workshop on Radon Monitoring in Radioprotection Environmental Radioactivity and Earth Sciences, ICTP, Trieste, World Scientific, Singapore, 1989, p. 244.

[219] M.M. Monnin, J.L. Seidel, Nucl. Instrum. Methods Phys. Res. A 314 (1992) 316.

[220] B. Zmazek, L. Todorovski, S. Dzeroski, J. Vaupotic, I. Kobal, Appl. Radiat. Isot. 58 (2003) 697.

[221] K. Ioannides, C. Papachristodoulou, K. Stamoulis, D. Karamanis, S. Pavlides, A. Chatzipetros, E. Karakala, Appl. Radiat. Isot. 59 (2003) 205.

[222] J. Planinic, V. Radolic, B. Vukovic, Nucl. Instrum. Methods Phys. Res. A, in press, on line at sciencedirect.com.

[223] N. Segovia, M. Mena, P. Peña, E. Tamez, J.L. Seidel, M. Monnin, C. Valdes, Radiat. Meas. 31 (1999) 307. 
[224] R.L. Fleischer, P.B. Price, R.M. Walker, Rev. Sci. Instrum. 34 (1963) 510.

[225] I. Lengar, J. Skvarc, R. Ilic, Radiat. Meas. 36 (2003) 115.

[226] M.P. Dhairyawan, P.K. Marathe, O.P. Massand, Radiat. Meas. 36 (2003) 435.

[227] E. Fantuzzi, B. Morelli, G. Falangi, L. Patrizii, V. Togo, Radiat. Prot. Dosim. 101 (2002) 573.

[228] K. Oda, H. Ichijo, N. Miyawaki, T. Yamauchi, Y. Nakane, Radiat. Meas. 34 (2001) 171.

[229] D. Hermsdorf, R. Bretschneider, B. Dorschel, J. Henniger, Radiat. Meas. 31 (1999) 431.

[230] R. Ilic, J. Skvarc, A.N. Golovchenko, Radiat. Meas. 36 (2003) 83.

[231] J. Ahrens, et al. Nucl. Instrum. Methods A 522 (2004) 347.

[232] P.B. Price, R.M. Walker, Nature 196 (1962) 732.

[233] P.B. Price, R.M. Walker, J. Geophys. Res. 68 (1963) 4847.

[234] M. Maurette, P. Pellas, R.M. Walker, Nature 204 (1964) 821.

[235] P.A.M. Dirac, Proc. R. Soc. A 133 (1931) 60.

[236] C. Soyland, S.P. Hassfjell, Radiat. Environ. Biophys. 39 (2000) 125.

[237] D. Selmeczi, B. Szabó, L. Sajo-Bohus, N. Rozlosnik, Radiat. Meas. 34 (2001) 549.

[238] B. Dorschel, D. Hermsdorf, S. Pieck, S. Starke, H. Thiele, F. Weickert, Nucl. Instrum. Methods Phys. Res. B 207 (2003) 154.

[239] B. Dorschel, D. Hermsdorf, S. Pieck, S. Starke, H. Thiele, Nucl. Instrum. Methods Phys. Res. B 187 (2002) 525. 